\title{
Probabilistic Analyses of \\ Combinatorial Optimization Problems on Random Shortest Path Metrics
}





\section{PROBABILISTIC ANALYSES OF COMBINATORIAL OPTIMIZATION PROBLEMS ON RANDOM SHORTEST PATH METRICS}

Stefan Klootwijk 



\title{
PROBABILISTIC ANALYSES OF COMBINATORIAL OPTIMIZATION PROBLEMS ON RANDOM SHORTEST PATH METRICS
}

\author{
PROEFSCHRIFT
}

ter verkrijging van

de graad van doctor aan de Universiteit Twente, op gezag van de rector magnificus, prof.dr.ir. A. Veldkamp, volgens besluit van het College voor Promoties in het openbaar te verdedigen op woensdag 3 november 2021 om 14:45 uur

door

Stefan Klootwijk

geboren op 22 augustus 1993

te Enschede, Nederland 
Dit proefschrift is goedgekeurd door de promotor:

Dr. B. Manthey

\section{UNIVERSITY | DIGITAL SOCIETY Digital Society Institute \\ OF TWENTE. INSTITUTE $\quad$ P.O. Box 217 \\ 7500 AE Enschede, The Netherlands}

This work is supported by the Netherlands Organization for Scientific Research (NWO), project number 613.001.402.

ISBN: 978-90-365-5249-3

ISSN: 2589-7721 (DSI Ph.D. Thesis Series No. 21-011)

DOI: $10.3990 / 1.9789036552493$

Available online at https://dx.doi.org/10.3990/1.9789036552493

Typeset in $\mathrm{LT}_{\mathrm{E}} \mathrm{X}$

Printed by Ipskamp Printing, Enschede

Cover design by Stefan Klootwijk

Copyright $@ 2021$, Stefan Klootwijk, Enschede, The Netherlands

All rights reserved. No parts of this thesis may be reproduced, stored in a retrieval system or transmitted in any form or by any means without permission of the author.

Alle rechten voorbehouden. Niets uit deze uitgave mag worden vermenigvuldigd, in enige vorm of op enige wijze, zonder voorafgaande schriftelijke toestemming van de auteur. 


\section{Promotiecommissie:}

Voorzitter \& secretaris: Prof.dr. J.N. Kok

University of Twente

Promotor:

Dr. B. Manthey

University of Twente

Leden:

Dr. R.P. Hoeksma

University of Twente

Prof.dr. R.W. van der Hofstad

Eindhoven University of Technology

Prof.dr. N.V. Litvak

University of Twente

Prof.dr. M.J. Uetz

University of Twente

Prof.dr. T. Vredeveld

Maastricht University 



\section{Contents}

1 Introduction 1

1.1 Probabilistic Analysis . . . . . . . . . . . . . . . . . . . 2

1.2 Combinatorial Optimization Problems . . . . . . . . . . . . . . 3

1.2.1 Minimum-Distance Perfect Matching Problem . . . . . . . 4

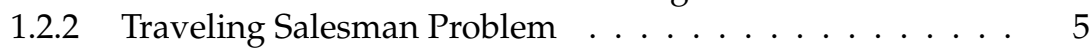

$1.2 .3 \quad k$-Median Problem $\ldots \ldots \ldots \ldots$. . . . . . . . . . . 7

1.2.4 Facility Location Problem . . . . . . . . . . . . . . . . . 8

1.2 .5 Steiner Tree Problem . . . . . . . . . . . . . . . . . . 9

1.3 General Notation and Conventions . . . . . . . . . . . . . . . 10

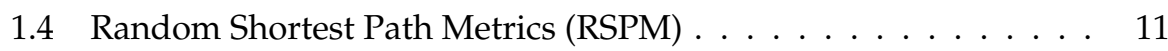

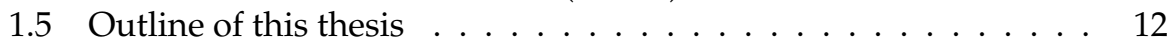

2 Properties of RSPM 15

2.1 Bounds for the Generalized Erlang Distribution . . . . . . . . . 16

2.2 Complete Graphs $\ldots \ldots \ldots \ldots \ldots \ldots \ldots$

2.3 General Graphs . . . . . . . . . . . . . . . . . . . . . . . . . . 21

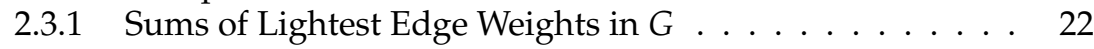

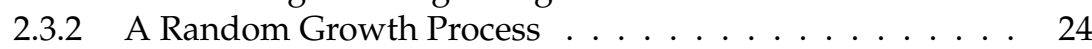

$2.3 .3 \quad$ Clustering . . . . . . . . . . . . . . . . 25

$3 \quad$ Analysis of Heuristics on RSPM from Dense ERRG Graphs 27

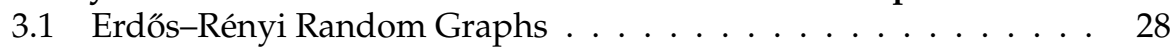

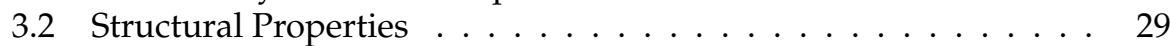

3.2 .1 A Random Growth Process . . . . . . . . . . . . . . . . 30

3.2 .2 Clustering . . . . . . . . . . . . . . . . . . 33

3.3 Analysis of Heuristics $\ldots \ldots \ldots \ldots \ldots$. . . . . . . . 34

3.3.1 Greedy Heuristic for Minimum-Distance Perfect Matching 35

3.3 .2 Nearest Neighbor Heuristic for TSP $\ldots \ldots \ldots \ldots$

3.3 .3 Insertion Heuristics for TSP . . . . . . . . . . . . . . . 37

3.3 .4 Running Time of 2-Opt Heuristic for TSP . . . . . . . . . 38

3.3.5 Trivial Heuristic for $k$-Median . . . . . . . . . . . . . . . 41

3.4 Application to the Erdős-Rényi Random Graph Model . . . . . . 46

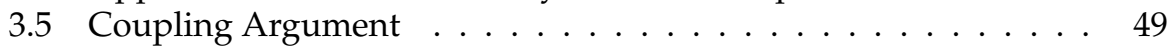


$4 \quad$ Analysis of Heuristics on RSPM from Sparse Graphs 55

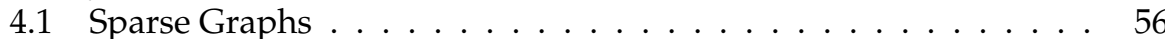

4.2 Structural Properties . . . . . . . . . . . . . . . . . . . . 57

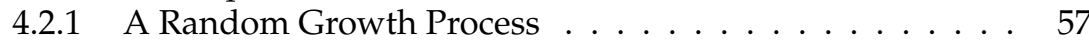

$4.2 .2 \quad$ Clustering and a Tail Bound for $\Delta_{\max } \ldots \ldots \ldots \ldots$. . . . 61

$4.3 \quad$ Analysis of Greedy-like Heuristics for Matching and TSP . . . . . 63

4.3.1 Greedy Heuristic for Minimum-Distance Perfect Matching 63

\begin{tabular}{|llll}
4.3 .2 & Nearest Neighbor Heuristic for TSP $\ldots \ldots \ldots$ & 64
\end{tabular}

4.3 .3 Insertion Heuristics for TSP . . . . . . . . . . . . . . . . . 65

4.4 Analysis of 2-Opt for TSP $\ldots \ldots \ldots \ldots \ldots$

$5 \quad$ Analysis of Facility Location on RSPM from Complete Graphs $\quad 69$

5.1 Additional notation $\ldots \ldots \ldots \ldots \ldots \ldots \ldots$

$5.2 \quad$ A Simple Heuristic and Some of Its Properties . . . . . . . . . 70

5.2.1 Probability Distribution of ALG . . . . . . . . . . . . 71

5.3 Technical Lemmas. . . . . . . . . . . . . . . . . . . . . . . . 71

5.4 Bounds for the Optimal Solution $\ldots \ldots \ldots \ldots \ldots \ldots$. . . . . . . 73

5.5 Main Results . . . . . . . . . . . . . . . . . . . . . . . . . . . 75

5.5 .1 Opening a few facilities . . . . . . . . . . . . . . 75

5.5 .2 Opening many facilities . . . . . . . . . . . . . 86

6 Minor Results on RSPM from Complete Graphs 97

6.1 Steiner Tree . . . . . . . . . . . . . . . . . . . . . . . 98

6.2 Numerical Analysis of 2-Opt … . . . . . . . . . . . . . . 99

$\begin{array}{lll}7 & \text { Concluding Remarks } & 103\end{array}$

7.1 Other Distributions . . . . . . . . . . . . . . . . . . . . . . . . . . 104

7.2 Future Work . . . . . . . . . . . . . . . . . . . . . . . . . . . . . . 104

\begin{tabular}{ll}
\hline Bibliography & 107
\end{tabular}

$\begin{array}{ll}\text { Summary } & 113\end{array}$

\begin{tabular}{ll}
\hline Samenvatting & 115
\end{tabular}

\begin{tabular}{ll}
\hline Dankwoord & 117
\end{tabular}

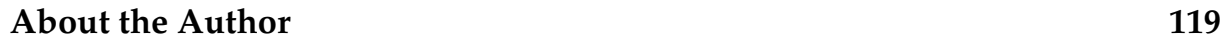


Chapter 1

\section{Introduction}




\subsection{Probabilistic Analysis}

Large-scale optimization problems, such as the traveling salesman problem, show up in many real-life applications. Many such problems concern some kind of distances: either actual physical distances, or some more abstract distance measure, for example travel times or transportation costs. In practical situations these distances commonly satisfy the triangle inequality: the distance from $A$ to $B$ is not larger than the distance from $A$ via $C$ to $B$.

Unfortunately, usually it is not possible to solve these optimization problems to optimality within a reasonable amount of time, especially when problem instances get larger. Therefore, in practice these kind of problems are often tackled by using approximation algorithms or ad-hoc heuristics. Somewhat surprisingly, these often quite simple ad-hoc heuristics succeed in finding closeto-optimal solutions within a reasonable amount of time. However, from a theoretical point of view, the worst-case performance of such heuristics is usually rather bad. One of the challenges within the analysis of algorithms is to better understand why these heuristics perform so well on practical problem instances.

It is not too difficult to see why the classical worst-case analysis often falls short of explaining the practical performance of (simple) heuristics: usually it uses some artificial worst-case instances that do not resemble any practical instances and do not show up in practice. In order to find theoretical results that are closer to the practical observations, probabilistic analysis has been widely used over the last decades. In probabilistic analysis the performance of algorithms is analysed with respect to random instances. One of the main challenges here is to come up with a good probabilistic model to generate these random instances: on the one hand this model should be sufficiently simple in order to make the probabilistic analysis possible, but on the other hand the model should reflect realistic instances.

In the 'early days' of probabilistic analysis, random instances where either embedded in Euclidean space or generated by using independent random edge lengths (e.g. [2, 5, 8, 18, 23, 25, 26, 37, 38, 58, 59]). Although these models have some nice mathematical properties that enable the probabilistic analyses, they have shortcomings regarding their realism. Instances generated by using independent random edge lengths do not satisfy the triangle inequality, whereas, as mentioned above, real-life instances usually do satisfy the triangle inequality. Also, the amount of applications where distances are actually Euclidean is rather limited, although Euclidean instances do satisfy the triangle inequality.

Given the considerations above, it seems natural to widen the scope of models for generating random instances. Recently, Bringmann et al. [15] did this by using the following model, already proposed by Karp and Steele in 1985 [39]: given an undirected complete graph, draw edge weights independently at random and then define the distance between any two vertices as the total weight of the shortest path between them, measured with respect to those random weights. Bringmann et al. called this model 'random shortest path metrics'. 
The model itself is not new. It is also known as first-passage percolation, originally introduced by Hammersley and Welsh as a model for fluid flow through a (random) porous medium [27, 32]. It has become one of the most classical models in probability theory. A lot of studies have been conducted on the model of random shortest path metrics, or first-passage percolation, itself. Among other things, these studies include questions related to the length of the shortest paths (e.g. [20, 28, 33|), to the number of edges on the shortest paths (e.g. [1, 29]), but also to the distribution of the total length of several combinatorial structures, such as minimal spanning trees and shortest path trees (e.g. [24, 30, 31]).

However, except for an answer from Dyer and Frieze [21] to a question posed by Karp and Steele [39], systematic research of the behaviour and performance of (simple) heuristics and approximation algorithms for combinatorial optimization problems on random instances generated by this model was initiated only recently in the aforementioned paper by Bringmann et al. [15].

In this thesis we look further into this model and into several variations of this model that improve the 'realism aspect' of the model, while still being sufficiently tractable for analysis. We focus on the probabilistic performance of some simple heuristics for several combinatorial optimization problems on random instances generated by these models.

\subsection{Combinatorial Optimization Problems}

In this thesis we consider the performance of heuristics for several combinatorial optimization problems: the minimum-distance perfect matching problem, the traveling salesman problem, $k$-median problem, the facility location problem, and the Steiner tree problem. These problems are among the best-known combinatorial optimization problems and have plenty of applications in real life. We introduce these five problems in this section.

The instances of all these problems are defined on a mathematical space $(V, d)$ consisting of a set of vertices $V$ and a distance function $d: V \times V \rightarrow \mathbb{R}_{\geq 0}$ that gives a distance between every pair of vertices in the set $V$. In general, any distance function $d$ can be used to define problem instances. However, following the arguments in the previous section, in this thesis we assume that the distance function is a so-called metric on $V$. Most importantly, this implies that the distance function is symmetric $(d(x, y)=d(y, x)$ for all $x, y \in V)$ and that the triangle inequality holds $(d(x, z) \leq d(x, y)+d(y, z)$ for all $x, y, z \in V)$. Nevertheless, observe that the problem definitions below do not require the distance function $d$ to be a metric.

The problems that we consider are often viewed as a graph problem on the complete graph with vertex set $V$. In this view, between each pair of vertices $u, v \in V$ there is an 'edge' with weight/length/distance equal to $d(u, v)$. In order to avoid potential confusion when a graph is involved in the construction of a (metric) space, we write quotation marks around these 'edges' of a (metric) space. 


\subsubsection{Minimum-Distance Perfect Matching Problem}

Matching problems comprise an important set of problems that link the areas of graph theory and combinatorial optimization. They arise in cases where we want to find some kind of 'optimal' pairing of the members of a set. In case of the minimum-distance perfect matching problem we are given a set of 'objects' and a 'distance' (or weight) for each pair of objects, and we want to match all objects into pairs such that the total distance is minimal.

Formally, we define the minimum-distance perfect matching problem as follows. Consider a (metric) space $(V, d)$ with $V=\left\{v_{1}, \ldots, v_{n}\right\}$, suppose that $n$ is even and let $E=\left\{e_{i j}:=\left\{v_{i}, v_{j}\right\}: 1 \leq i<j \leq n\right\}$ denote the set of 'edges' of this (metric) space. A matching $M$ is a subset of the 'edges' $E$ such that each vertex in $V$ is incident to at most one 'edge' in $M$. If each vertex in $V$ is incident to exactly one 'edge' in $M$, then $M$ is called a perfect matching. The total distance $d(M)$ of a matching is given by

$$
d(M)=\sum_{e_{i j} \in M} d\left(v_{i}, v_{j}\right)
$$

An optimal solution $M^{*}$ to the minimum-distance perfect matching problem is given by a matching $M$ for which the total distance is minimal among all perfect matchings, or, equivalently,

$$
M^{*}:=\operatorname{argmin}\{d(M): M \text { is a perfect matching }\} .
$$

The minimum-distance perfect matching problem can be solved to optimality in $O\left(n^{3}\right)$ time [47]. However, in practice this is often too slow, especially if the number of vertices is large. Therefore, people often rely on (simple) heuristics to solve this problem in practical situations. In this thesis we only consider the greedy heuristic for the minimum-distance perfect matching problem, which is arguably the simplest heuristic for this problem.

Greedy. The greedy heuristic starts with an empty matching and iteratively adds a pair of currently unmatched vertices (an 'edge') to the matching such that the distance between them is minimal (among all pairs of currently unmatched vertices). It is known that the worst-case approximation ratio for this heuristic on metric instances is $O\left(n^{\log _{2}(3 / 2)}\right) \approx O\left(n^{0.58}\right)$ [54]. Moreover, for random Euclidean instances, the greedy heuristic has an approximation ratio of $O(1)$ with high probability [6]. For instances with independent edge lengths (thus not necessarily metric), the greedy heuristic returns a matching with an expected distance of $\Theta(\ln (n))[5]$ and the optimal matching has a total distance of $\Theta(1)$ with high probability [59], which gives an approximation ratio of $O(\ln (n))$.

For random shortest path metrics generated from complete graphs the expected approximation ratio of the greedy heuristic is $O(1)$ [15]. In this thesis, we show that a similar result holds for random shortest path metrics generated 
from (dense) Erdős-Rényi random graphs (Section 3.4 and for random shortest path metrics generated from sparse graphs with fast growing cut sizes (Section 4.3.1.

\subsubsection{Traveling Salesman Problem}

The traveling salesman problem is one of the most intensively studied problems in combinatorial optimization. Given $n$ cities with distances between them, the traveling salesman problem asks for the shortest tour through all the cities such that each city is visited exactly once, and the tour ends in the starting city.

Formally, we define the traveling salesman problem as follows. Consider a (metric) space $(V, d)$ with $V=\left\{v_{1}, \ldots, v_{n}\right\}$ and let $E=\left\{e_{i j}:=\left\{v_{i}, v_{j}\right\}\right.$ : $1 \leq i<j \leq n\}$ denote the set of 'edges' of this (metric) space. A TSP tour $T$ is a Hamiltonian cycle in $(V, E)$, i.e., a subset of the 'edges' $E$ such that $T=$ $\left\{e_{\pi(1) \pi(2)}, e_{\pi(2) \pi(3)}, \ldots, e_{\pi(n) \pi(1)}\right\}$ for some permutation $\pi$ of $[n]$. The total distance $d(T)$ of a TSP tour is given by

$$
d(T)=\sum_{e_{i j} \in T} d\left(v_{i}, v_{j}\right) .
$$

An optimal solution $T^{*}$ to the traveling salesman problem is given by a TSP tour $T$ for which the total distance is minimal among all TSP tours, or, equivalently,

$$
T^{*}:=\operatorname{argmin}\{d(T): T \text { is a TSP tour }\} .
$$

The traveling salesman problem is $\mathcal{N} \mathcal{P}$-hard, even if we restrict ourselves to metric instances [45]. It is perhaps the most-studied $\mathcal{N} \mathcal{P}$-hard combinatorial optimization problem, and over the last decades almost every (meta)heuristic has been applied to it. In this thesis we consider three of these heuristics for the traveling salesman problem: two greedy-like construction heuristics (the nearest neighbor heuristic and the insertion heuristics) and one local search heuristic (2-opt).

Nearest neighbor. The nearest neighbor heuristic is a greedy-like tour construction heuristic. It starts with an arbitrary vertex as its current vertex and iteratively builds a TSP tour by traveling from its current vertex to the closest unvisited vertex and adding the corresponding 'edge' to the tour (and at the end closing the tour by going back to its first vertex after all vertices have been visited). It is known that the worst-case approximation ratio for this heuristic on metric instances is $O(\ln (n))$ [56]. Moreover, for random Euclidean instances, the nearest neighbor heuristic has an approximation ratio of $O(1)$ with high probability [8]. For instances with independent edge lengths (thus not necessarily metric), the nearest neighbor heuristic returns a TSP tour with an expected 
length of $H_{n-1}=\Theta(\ln (n))^{冈}$ while the optimal TSP tour has a total length of $\Theta(1)$ with high probability [25], which gives an approximation ratio of $O(\ln (n))$.

For random shortest path metrics generated from complete graphs the expected approximation ratio of the nearest neighbor heuristic is $O(1)$ [15]. In this thesis, we show that a similar result holds for random shortest path metrics generated from (dense) Erdős-Rényi random graphs (Section 3.4) and for random shortest path metrics generated from sparse graphs with fast growing cut sizes (Section 4.3.2).

Insertion. The insertion heuristics are a class of greedy-like construction heuristics. An insertion heuristic starts with an initial optimal tour on a few vertices that are selected according to some predefined rule $R$, and iteratively chooses (according to the same rule $R$ ) a vertex that is not in the tour yet and inserts this vertex in the current tour such that the total distance of the tour increases the least. Usually the rule $R$ prescribes that the initial tour is just some tour on three vertices or an edge (i.e., a tour on two vertices) or even a single vertex. Examples of rules used for choosing a vertex to insert in the tour are 'nearest insertion' (choose the vertex that has the shortest distance to vertex already in the tour), 'farthest insertion' (choose the vertex whose minimal distance to a vertex already in the tour is maximal) and 'cheapest insertion' (choose the vertex whose insertion causes the smallest increase in the length of the tour) [53].

It is known that the worst-case approximation ratio for this heuristic for any rule $R$ on metric instances is $O(\ln (n))$ [56]. For some specific rules $R$, this worstcase approximation ratio can be improved to a constant: for example, nearest insertion and cheapest insertion have a worst-case approximation ratio on metric instances of $2-2 / n[56]$. Moreover, for random Euclidean instances, some insertion rules $R$ have an approximation ratio of $\Omega(\ln (n) / \ln \ln (n))$ |7|. For random shortest path metrics generated from complete graphs the expected approximation of the insertion heuristic is $O(1)$ for any rule $R$ [15]. In this thesis, we show that a similar result holds for random shortest path metrics generated from (dense) Erdős-Rényi random graphs (Section 3.4) and for random shortest path metrics generated from sparse graphs with fast growing cut sizes (Section 4.3.3.

2-opt. The 2-opt heuristic is probably the most famous simple local search heuristic for the traveling salesman problem. It starts with an initial solution and iteratively improves this solution by applying so-called 2-exchanges until no improvement is possible anymore by any 2-exchange. In a 2-exchange the heuristic takes two 'edges' $\left\{v_{1}, v_{2}\right\}$ and $\left\{v_{3}, v_{4}\right\}$, where $v_{1}, v_{2}, v_{3}, v_{4}$ are visited in this order in the current solution, and replaces them by the two 'edges' $\left\{v_{1}, v_{3}\right\}$

*The expected distance of the first added 'edge' is equal to the minimum of $n-1$ independent edge weights, and thus has an expected length of $1 /(n-1)$. In general, the expected distance of the $k$-th added 'edge' is equal to the minimum of $n-k$ independent edge weights, and thus has an expected length of $1 /(n-k)$. 
and $\left\{v_{2}, v_{4}\right\}$ to obtain a new solution. The improvement of this 2-exchange is $\delta=d\left(v_{1}, v_{2}\right)+d\left(v_{3}, v_{4}\right)-d\left(v_{1}, v_{3}\right)-d\left(v_{2}, v_{4}\right)$. A solution is called 2-optimal if $\delta \leq 0$ for all possible 2-exchanges.

The actual performance of the 2-opt heuristic strongly depends on the choice of the initial solution and the sequence of improvements. In this thesis we look at the worst possible outcome of the 2-opt heuristic, as others have been doing before (see e.g. [18, 22]), since this decouples the actual heuristic from the initialization and therefore keeps the analysis tractable.

It is known that the worst-case approximation ratio for this heuristic for any rule $R$ on metric instances is $O(\sqrt{n})$ [18]. Moreover, for random Euclidean instances, the 2-opt heuristic has an expected approximation ratio of $O(1)$ [18]. For instances with independent edge lengths (thus not necessarily metric), the 2-opt heuristic has an expected approximation ratio of $O(\sqrt{n \ln (n)})$ [58]. For random shortest path metrics generated from complete graphs the expected approximation ratio of the 2-opt heuristic is $O(\ln (n))$, but it is an open problem whether this (almost) trivial bound can be improved or not [15]. In this thesis we present some numerical results that seem to imply that the expected approximation ratio (of the worst possible outcome of the 2-opt heuristic) might indeed be $\Theta(\ln (n))$ for random shortest path metrics generated from complete graphs (Section 6.2). We also show that the expected approximation ratio of the 2-opt heuristic is $O(1)$ for random shortest path metrics generated from sparse graphs (Section 4.4).

Unlike the nearest neighbor and insertion heuristics which always run in polynomial time, the 2-opt heuristic uses an exponential number of iterations in the worst-case scenario. However, for random Euclidean instances, the 2-opt heuristic uses only an expected number of $O\left(n^{10} \ln (n)\right)$ iterations [18]. For random shortest path metrics generated from complete graphs the expected number of iterations of the 2-opt heuristic is bounded by $O\left(n^{8} \ln ^{3}(n)\right)[15$. In this thesis, we show that a similar result holds for random shortest path metrics generated from (dense) Erdős-Rényi random graphs (Section 3.4) and that a slightly weaker result holds for random shortest path metrics generated from arbitrary (connected) graphs (Section 3.3.4).

\subsection{3 $k$-Median Problem}

The $k$-median problem, also known as the $p$-median or $k$-medoids problem, is a discrete location problem. Given $n$ locations with distances between them, it asks us to open a facility at exactly $k$ out of these $n$ locations in such a way that the total 'connection costs' of all locations is minimal.

Formally, we define the $k$-median problem as follows. Consider a (metric) space $(V, d)$ with $V=\left\{v_{1}, \ldots, v_{n}\right\}$ and let $E=\left\{e_{i j}:=\left\{v_{i}, v_{j}\right\}: 1 \leq i<j \leq n\right\}$ denote the set of 'edges' of this (metric) space. The objective of the $k$-median problem is to find a subset of the vertices $U \subseteq V$ of size $k$ such that the total 
'connection costs' for the other vertices

$$
\operatorname{cost}(U)=\sum_{v \in V} \min _{u \in U} d(v, u)
$$

is minimal among all subsets $U$ of size $k$. In other words, an optimal solution $U^{*}$ to the $k$-median problem is given by

$$
U^{*}:=\operatorname{argmin}\{\operatorname{cost}(U): U \subseteq V,|U|=k\} .
$$

The $k$-median problem is $\mathcal{N} \mathcal{P}$-hard [35]. The currently best known polynomialtime approximation algorithm for this problem achieves a (worst-case) approximation ratio of $2.611+\varepsilon[17]$. In this thesis we only consider a trivial heuristic for the $k$-median problem.

Trivial. The trivial heuristic for the $k$-median problem that we consider in this thesis simply picks $k$ vertices independent of the metric space, e.g., $U=$ $\left\{v_{1}, \ldots, v_{k}\right\}$. The worst-case approximation ratio of this heuristic is unbounded, even if we restrict ourselves to metric instances. However, for random shortest path metrics generated from complete graphs the expected approximation ratio of this trivial heuristic is $1+o(1)$ for $k$ sufficiently small and $O(1)$ for larger (but not too large) $k$ [15]. In this thesis, we show that a similar result holds for random shortest path metrics generated from (dense) Erdős-Rényi random graphs (Section 3.4).

\subsubsection{Facility Location Problem}

Facility location problems deal with the question of where to locate certain facilities so that the total cost of opening the facilities and transportation to the locations without a facility is minimized. We consider the uncapacitated facility location problem, in which there are no constraints on the amount of locations that can be 'connected' to an open facility.

Formally, we define the (uncapacitated) facility location problem as follows. Consider a (metric) space $(V, d)$ with $V=\left\{v_{1}, \ldots, v_{n}\right\}$ and let $E=\left\{e_{i j}:=\right.$ $\left.\left\{v_{i}, v_{j}\right\}: 1 \leq i<j \leq n\right\}$ denote the set of 'edges' of this (metric) space. Moreover, for each vertex $v_{i} \in V$, let $f_{i}>0$ denote the so-called opening cost of a facility at vertex $v_{i}$. The objective of the facility location problem is to find a non-empty subset $U \subseteq V$ such that the total cost

$$
c(U)=f(U)+\sum_{v \in V} \min _{u \in U} d(v, u)
$$

is minimal among all non-empty subsets $U \subseteq V$, where $f(U)$ denote the total opening cost of facilities at all vertices in $U$. In other words, an optimal solution 
$U^{*}$ to the facility location problem is given by

$$
U^{*}=\operatorname{argmin}\{c(U): \varnothing \neq U \subseteq V\} .
$$

The facility location problem is $\mathcal{N} \mathcal{P}$-hard [19]. The currently best known polynomial-time approximation algorithm for this problem achieves a (worst-case) approximation ratio of 1.488 [48]. Flaxman et al. conducted a probabilistic analysis for the facility location problem on Euclidean instances [23]. They expected to prove that some polynomial-time approximation algorithms (including the at that point best known approximation algorithm [50]) would be asymptotically optimal under these circumstances, but found out that this is not the case. On the other hand, they described a trivial heuristic which is asymptotically optimal in the Euclidean model.

In Chapter 5 we conduct a probabilistic analysis for the facility location problem on random shortest path metrics generated from complete graphs. We will investigate a rather simple heuristic that opens the $\kappa$ cheapest facilities (breaking ties arbitrarily), where $\kappa$ only depends on the number of vertices and the facility opening costs $f_{i}$. We show that this simple heuristic is asymptotically optimal for many opening costs $f_{i}$, and provide a bound for the approximation ratio in the remaining cases.

\subsubsection{Steiner Tree Problem}

The Steiner tree problem can be seen as a generalization of the shortest path problem and the minimum spanning tree problem. Given a set of points in a (discrete metric) space, the Steiner tree problem asks us to find a shortest network that connects all the points in this set. If the set only contains two points, then the problem is equivalent to the shortest path problem, and if the set contains all points of the space, then the problem is equivalent to the minimum spanning tree problem.

Formally, we define the Steiner tree problem as follows. Consider a (metric) space $(V, d)$ with $V=\left\{v_{1}, \ldots, v_{n}\right\}$ and let $E=\left\{e_{i j}:=\left\{v_{i}, v_{j}\right\}: 1 \leq i<j \leq n\right\}$ denote the set of 'edges' of this (metric) space. Moreover, let $R \subseteq V$ be the set of so-called terminals, or 'required' vertices. A Steiner tree $T=(U, F)$ is a subgraph of the complete graph induced by the (metric) space such that $R \subseteq$ $U \subseteq V$ and $T$ is a tree. The total distance $d(T)$ of a Steiner tree is given by

$$
d(T)=\sum_{e_{i j} \in F} d\left(v_{i}, v_{j}\right) .
$$

An optimal solution $T^{*}$ to the Steiner tree problem is given by a Steiner tree $T$ for which the total distance is minimal among all Steiner trees, or, equivalently,

$$
T^{*}:=\operatorname{argmin}\{d(T): T \text { is a Steiner tree }\} .
$$


The Steiner tree problem is $\mathcal{N} \mathcal{P}$-hard in general [36], although some special cases are solvable in polynomial time (most notably the cases with $|R|=2$ (shortest path) or $R=V$ (minimum spanning tree)). The currently best known polynomial-time approximation algorithm for this problem achieves a (worstcase) approximation ratio of $\ln (4)+\varepsilon \approx 1.39$ [16]. In this thesis we consider a simpler 2-approximation algorithm.

This simple heuristic computes a minimum spanning tree on the subspace induced by $R$ and returns it as a solution, completely disregarding the vertices in $V \backslash R$. It is known that the worst-case approximation ratio for this heuristic on metric instances is $2-2 /|R|$. For non-metric instances, a slightly adjusted version of this heuristic also obtains a worst-case approximation ratio of $2-$ $2 /|R|[46]$.

For instances with independent edge lengths (thus not necessarily metric), the slightly adjusted simple heuristic is asymptotically optimal if $|R|=o(n)$ [12]. In this thesis, we extend this result to show that this simple heuristic also is asymptotically optimal for random shortest path metrics generated from complete graphs if $|R|=o(n)$ (Section 6.1).

\subsection{General Notation and Conventions}

In this section we present some general notation and conventions that we use throughout this thesis. For $n \in \mathbb{N}$, we use $[n]$ as shorthand notation for the set $\{1, \ldots, n\}$. Sometimes we use $\exp (\cdot)$ to denote the exponential function with base $e$.

We denote by $X \sim P$ that a random variable $X$ is distributed according to a probability distribution $P . \operatorname{Exp}(\lambda)$ denotes the exponential distribution with parameter $\lambda$. We write $X \sim \sum_{i=1}^{n} \operatorname{Exp}\left(\lambda_{i}\right)$ if $X$ is the sum of $n$ independent exponentially distributed random variables having parameters $\lambda_{1}, \ldots, \lambda_{n}$. In particular, $X \sim \sum_{i=1}^{n} \operatorname{Exp}(\lambda)$ denotes an Erlang distribution with parameters $n$ and $\lambda$. If a random variable $X_{1}$ is stochastically dominated by another random variable $X_{2}$, i.e., if we have $\mathbb{P}\left(X_{1} \leq x\right) \geq \mathbb{P}\left(X_{2} \leq x\right)$ for all $x$, we denote this by $X_{1} \precsim X_{2}$. If $X_{1}, \ldots, X_{n}$ are $n$ random variables, then $X_{(1)}, \ldots, X_{(n)}$ are the order statistics corresponding to $X_{1}, \ldots, X_{n}$, i.e., $X_{(i)}$ is the $i$ th smallest value among $X_{1}, \ldots, X_{n}$ for all $i \in[n]$.

Furthermore, we use $H_{n}^{(m)}$ as shorthand notation for the $n$th generalized harmonic number of order $m$, i.e., $H_{n}^{(m)}=\sum_{i=1}^{n} 1 / i^{m}$. We use $H_{n}=H_{n}^{(1)}$ for simplicity. Observe that for $m \in(0,1)$ we can view the generalized harmonic numbers as Riemann sums for $\int 1 / x^{m} \mathrm{~d} x$ and bound them as follows:

$$
\frac{(n+1)^{1-m}-1}{1-m} \leq H_{n}^{(m)} \leq \frac{(n+1)^{1-m}}{1-m} .
$$


Regarding summations, if $a$ and / or $b$ is non-integer, then we use the convention $\sum_{i=a}^{b} f(i)=\sum_{i=\lceil a\rceil}^{\lfloor b\rfloor} f(i)$. We also adopt the convention that $\sum_{i=a}^{b} f(i)=0$ if $a>b$ (or actually, if $\lceil a\rceil>\lfloor b\rfloor$ ).

Finally, we summarize the (commonly used) asymptotic notation that we use in the analysis of algorithms. Let $f(n)$ and $g(n)$ be non-negative real-valued functions defined on the natural numbers. We write $f(n)=O(g(n))$ if there are constants $c, n_{0}>0$ such that $f(n) \leq c \cdot g(n)$ for all $n \geq n_{0}$. We write $f(n)=\Omega(g(n))$ if there are constants $c, n_{0}>0$ such that $f(n) \geq c \cdot g(n)$ for all $n \geq n_{0}$. We write $f(n)=\Theta(g(n))$ if there are constants $c_{1}, c_{2}, n_{0}>0$ such that $c_{1} \cdot g(n) \leq f(n) \leq c_{2} \cdot g(n)$ for all $n \geq n_{0}$, or, equivalently, if $f(n)=O(g(n))$ and $f(n)=\Omega(g(n))$. Moreover, we write $f(n)=o(g(n))$ if $\lim _{n \rightarrow \infty} f(n) / g(n)=0$ and we write $f(n)=\omega(g(n))$ if $\lim _{n \rightarrow \infty} f(n) / g(n)=\infty$.

\subsection{Random Shortest Path Metrics (RSPM)}

In this thesis we consider random instances for our combinatorial problems using the model of random shortest path metrics. As already mentioned in Section 1.1. this model is also known as first-passage percolation, originally introduced by Hammersley and Welsh as a model for fluid flow through a (random) porous medium [27, 32].

Formally, we define random shortest path metrics as follows. Given an undirected simple graph $G=(V, E)$ on $n$ vertices, its corresponding random shortest path metric is constructed as follows. First, for each edge $e \in E$, we draw a random edge weight $w(e)$ independently according to the exponential distribution with parameter 1. (Exponential distributions are technically easiest to handle since they are memoryless. We briefly discuss other distributions in Chapter 7) Then, we define the distance function $d: V \times V \rightarrow \mathbb{R}_{\geq 0}$ as follows: for each $u, v \in V, d(u, v)$ is the total weight of a lightest $u, v$-path in $G$ (w.r.t. the random weights $w(\cdot))$. For completeness, if no such path exists, we set $d(u, v)=\infty$. Observe that this definition immediately implies that $d(v, v)=0$ for all $v \in V$, that $d(u, v)=d(v, u)$ for all $u, v \in V$, and that $d(u, v) \leq d(u, s)+d(s, v)$ for all $u, s, v \in V$.

We call the distance function $d(\cdot, \cdot)$ obtained by this process a random shortest path metric generated from $G$. Note that even though the graph $G$ does not need to be a complete graph, the metric $d(\cdot, \cdot)$ is always complete in the sense that between each pair of vertices $u, v \in V$ it has an 'edge' of distance $d(u, v)$. If $G=K_{n}$ (the complete graph on $n$ vertices), then the random shortest path metric generated from $G$ is equivalent to the random shortest path metric as defined by Bringmann et al. [15].

A lot of studies have been conducted on the model of random shortest path metrics (or first-passage percolation). Many of these studies focused on firstpassage percolation defined on the lattice $\mathbb{Z}^{d}$. The survey by Auffinger et al. |3] 
contains a thorough overview of research on this model. Other studies focused on first-passage percolation defined on complete graphs (e.g. [1, 20, 24. 28. 30, 31, 33]), first-passage percolation defined on Erdős-Rényi random graphs (e.g. [10. 29]), or first-passage percolation defined on other random graph models (e.g. [9, 11, 44]). In Chapter 22we elaborate on some of the results from these studies which are relevant for this thesis.

In this thesis, we use the following notation to denote some properties of these random shortest path metrics generated from $G=(V, E)$. The diameter of the random metric is denoted by $\Delta_{\max }:=\max _{u, v} d(u, v)$. Note that $\Delta_{\max }<\infty$ if and only if $G$ is connected. The $\Delta$-ball around a vertex $v, B_{\Delta}(v):=\{u \in V$ : $d(u, v) \leq \Delta\}$, is the set of vertices within distance $\Delta$ of $v$. Let $\pi_{k}(v)$ denote the $k$ th closest vertex from $v$ (including $v$ itself and breaking ties arbitrarily). Note that $\pi_{1}(v)=v$ for all $v \in V$. The distance from a vertex $v$ to this $k$ th closest vertex from it is denoted by $\tau_{k}(v):=d\left(v, \pi_{k}(v)\right)=\min \left\{\Delta:\left|B_{\Delta}(v)\right| \geq k\right\}$. Slightly abusing notation, we let $B_{\tau_{k}(v)}(v):=\left\{\pi_{i}(v): i=1, \ldots, k\right\}$ denote the set of the $k$ closest vertices to $v$ (including $v$ itself). The size of the cut in $G$ induced by this set, which plays an important role in some of the analyses in this thesis, is denoted by $\chi_{k}(v):=\left|\delta\left(B_{\tau_{k}(v)}(v)\right)\right|$, where $\delta(U):=\{\{u, v\} \in E: u \in U, v \notin U\}$ denotes the cut induced by $U$.

\subsection{Outline of this thesis}

The remainder of this thesis is organized as follows. In Chapter 2 we take a look at some mathematical properties of random shortest path metrics, focusing on the distribution of the distances between vertices in these random metric spaces. We start by mentioning some already known basic properties of the distances between vertices in random shortest path metrics generated from complete graphs. Then we extend these results to random shortest path metrics generated from arbitrary graphs. The most important result in this chapter is the generalization of a clustering result from Bringmann et al. [15], which is important for (some of) the results in Chapters 3 and 4 .

Chapters 3, 4 and 5 contain the probabilistic analyses that form the backbone of this thesis. In Chapter 3 the goal was to conduct some probabilistic analyses for optimization problems on random shortest path metrics generated from non-complete graphs. Our first step in this direction was the usage of (dense) Erdős-Rényi random graphs to generate the random shortest path metrics. We consider the greedy-like heuristics that we introduced in Sections 1.2.1, 1.2.2 and 1.2.3 for the minimum-distance perfect matching problem, the traveling salesman problem and the $k$-median problem, and we show that they all yield a constant expected approximation ratio in this model. These results can be seen as a generalization of the results from Bringmann et al. [15]. We also provide a coupling argument that shows that many results obtained for random shortest path metrics generated from complete graphs also hold for random shortest 
path metrics generated from dense Erdős-Rényi random graphs. This chapter is based on [42. 43].

In Chapter 4 we continue this line of research by investigating random shortest path metrics generated from sparse graphs for our probabilistic analyses. Intuitively, such random metric spaces are more 'realistic' than random shortest path metrics generated from dense graphs, since most real life networks are sparse. Initially, the research for this chapter focussed on random shortest path metrics generated from a specific class of sparse graphs: (finite square) grid graphs. Later on, we generalized the obtained results to random shortest path metrics generated from sparse graphs with fast growing cut sizes. We consider the heuristics that we introduced in Sections 1.2.1 and 1.2.2 for the minimumdistance perfect matching problem and the traveling salesman problem, and we show that they all yield a constant expected approximation ratio in this model. This chapter is based on [41].

In Chapter 5 we return to random shortest path metrics generated from complete graphs. We investigate the probabilistic properties of the rather simple heuristic for the facility location problem on this model that we introduced in Section 1.2.4 We show that this heuristic is asymptotically optimal if the facility opening costs are such that the number of facilities that are being opened by the heuristic is not too large $(\kappa=o(n))$, and also provide an upper bound for the expected approximation ratio in case the facility opening costs are such that the number of facilities being opened by the heuristic is large $(\kappa=\Theta(n))$. This chapter is based on [40].

In Chapter 6 we mention two somewhat smaller results that emerged during our research. The first result concerns the simple heuristic for the Steiner tree problem that we introduced in Section 1.2.5. We extend a known result to show that this heuristic is asymptotically optimal for random shortest path metrics generated from complete graphs if the number of terminals is not too large $(|R|=o(n))$. For the second result we present some numerical results concerning the 2-opt heuristic for the traveling salesman problem on random shortest path metrics generated from complete graphs. Bringmann et al. [15] remarked that it is (almost) trivial to see that the 2-opt heuristic has an expected approximation ratio of $O(\ln (n))$ in this model, and posed the question whether this could be improved to $o(\ln (n))$. Our numerical data seems to suggest that the expected approximation ratio of 2-opt on random shortest path metrics generated from complete graphs might actually be $\Theta(\ln (n))$.

Finally, Chapter 7 contains some concluding remarks. Among other things, we briefly discuss the usage of other probability distributions for generating random shortest path metrics. 



\section{Chapter 2}

\section{Properties of RSPM}

Before starting our probabilistic analyses of combinatorial optimization problems on random shortest path metrics we need to get a feeling for the structure of random shortest path metrics. When analysing random shortest path metrics, sooner or later we encounter sums of independently distributed exponential random variables, or, equivalently, random variables with a generalized Erlang distribution. Therefore, we start this chapter by stating some helpful results that enable us to manipulate this distribution.

After that, we have a look at some basic properties of random shortest path metrics generated from complete graphs. As others have done before, we use a growth process to derive the distribution of the distance between two arbitrarily chosen vertices. Using this distribution, we derive exact expressions for the expectation and variance of the distance between two arbitrarily chosen vertices, and the covariance between the distances of two adjacent 'edges' in this model.

For other properties of random shortest path metrics, we immediately consider the model using arbitrary graphs. We start with some useful results regarding the distribution of sums of lightest edge weights in arbitrary graphs. Then we generalize the growth process used to derive the distribution of distances in random shortest path metrics generated from complete graphs. Using this generalization we derive stochastic bounds for the distribution of distances in random shortest path metrics generated from arbitrary graphs.

We end this chapter with a general clustering result. This is a generalization of the clustering result for random shortest path metrics generated from complete graphs by Bringmann et al. [15]. Specific versions of this general clustering result form a crucial ingredient for (some of) the probabilistic analyses in the following chapters. 


\subsection{Bounds for the Generalized Erlang Distribution}

In this chapter, we come across some results containing random variables with a generalized Erlang distribution, also known as the hypoexponential distribution. This distribution represents the sum of independently distributed exponential random variables with different parameters. In this section, we state some helpful results that enable us to manipulate this distribution in a meaningful way. We start with two tail bounds for the probability distribution of the generalized Erlang distribution.

Lemma 2.1 ([34. Thm. 5.1(i, iii)]). Let $X \sim \sum_{i=1}^{m} \operatorname{Exp}\left(a_{i}\right)$. Moreover, let $\mu=$ $\mathbb{E}[X]=\sum_{i=1}^{m} 1 / a_{i}$ and $a_{*}=\min _{i} a_{i}$.

(i) For any $\lambda \geq 1$,

$$
\mathbb{P}(X \geq \lambda \mu) \leq \lambda^{-1} \exp \left(-a_{*} \mu(\lambda-1-\ln (\lambda))\right) .
$$

(ii) For any $\lambda \leq 1$,

$$
\mathbb{P}(X \leq \lambda \mu) \leq \exp \left(-a_{*} \mu(\lambda-1-\ln (\lambda))\right) .
$$

According to Janson, these bounds roughly show that the tail probabilities of $X$ decrease at about the same rate as the tail probabilities of $\operatorname{Exp}\left(a_{*}\right)$, i.e., as for the exponential variable with smallest $a_{i}$ and thus the fattest tail [34]. We can use the second tail bound to obtain a general upper bound on the cumulative distribution function of the generalized Erlang distribution. Note that this bound is somewhat weaker than the original tail bound. However, it is still sufficiently strong for our purposes and easier to use.

Corollary 2.2. Let $X \sim \sum_{i=1}^{m} \operatorname{Exp}\left(a_{i}\right)$. Moreover, let $\mu=\mathbb{E}[X]=\sum_{i=1}^{m} 1 / a_{i}$ and $a_{*}=\min _{i} a_{i}$. For any $x>0$,

$$
\mathbb{P}(X \leq x) \leq \exp \left(a_{*} \mu(1+\ln (x / \mu))\right)=\left(\frac{e x}{\mu}\right)^{a_{*} \mu} .
$$

Proof. Let $\lambda:=x / \mu$. If $\lambda \leq 1$, the result is a weaker version of Lemma 2.1(ii). If $\lambda>1$, then $1+\ln (x / \mu)>0$ and hence $\mathbb{P}(X \leq x) \leq 1<\exp \left(a_{*} \mu(1+\right.$ $\ln (x / \mu)))$.

Besides having (tail) bounds on the distribution function of the generalized Erlang distribution, sometimes we also want to bound the distribution itself. The following lemma enables us to 'bound' a generalized Erlang distribution by a 'regular' Erlang distribution (i.e. a sum of independently distributed exponential random variables where all parameters are equal) in the sense that the generalized Erlang distribution stochastically dominates a 'regular' Erlang distribution. 
Lemma 2.3 ([14, Thm. 2(ii)]). Let $X \sim \sum_{i=1}^{m} \operatorname{Exp}\left(\lambda_{i}\right)$ and $Y \sim \sum_{i=1}^{m} \operatorname{Exp}(\eta)$. Then

$$
X \succsim Y \quad \text { if and only if } \quad \prod_{i=1}^{m} \lambda_{i} \leq \eta^{m} .
$$

\subsection{Complete Graphs}

Within the framework of random shortest path metrics, random shortest path metrics generated from complete graphs are arguably the easiest to handle from a theoretical perspective. Most notably, the symmetry and regularity of the complete graph enable us to derive some exact results. In this section we derive exact expressions for the expectation and variance of the distance between two arbitrarily chosen vertices, and the covariance between the distances of two adjacent 'edges'. The expectation was already known (e.g. [15, 20, 33]) and is only included fo completeness. We extend this with the variance (although the asymptotic value of the variance was already known [33]) and the covariance.

We start by deriving the distribution of the distances in random shortest path metrics generated from complete graphs. The key ingredient for this derivation is the following growth process. This process has been analysed by, among others, Davis and Prieditis [20], Janson [33], and Bringmann et al. [15]. We repeat their analysis for completeness.

The values of $\tau_{k}(v)$ are generated by a birth process as follows. For $k=1$ we have $\tau_{k}(v)=0$. For $k \geq 1$ we can obtain $\tau_{k+1}(v)$ from $\tau_{k}(v)$ by looking at all edges that 'leave' $B_{\tau_{k}}(v)$, i.e., edges $(u, x)$ with $u \in B_{\tau_{k}}(v)$ and $x \notin B_{\tau_{k}}(v)$. Since we consider random shortest path metrics generated from complete graphs and since $\left|B_{\tau_{k}}(v)\right|=k$, there are $k(n-k)$ such edges. Moreover, by definition of $\tau_{k}(v)$, these edges are all conditioned to have a weight of at least $\tau_{k}(v)-d(v, u)$ (otherwise we would have $d(v, x) \leq d(v, u)+d(u, x)<\tau_{k}(v)$ ). Using the memorylessness of the exponential distribution, it follows that $\tau_{k+1}(v)-\tau_{k}(v)$ is the minimum of $k(n-k)$ exponential random variables (with parameter 1), or, equivalently $\delta_{k}:=\tau_{k+1}(v)-\tau_{k}(v) \sim \operatorname{Exp}(k(n-k))$. Again using the memorylessness of the exponential distribution, we can see that $\delta_{1}, \ldots, \delta_{n-1}$ are independent. Hence, we obtain $\tau_{k}(v)=\sum_{i=1}^{k-1} \delta_{i} \sim \sum_{i=1}^{k-1} \operatorname{Exp}(i(n-i))$.

Let $u, v \in V$ be two arbitrarily chosen distinct vertices. Observe that we can write

$$
d(u, v) \sim \tau_{N+1}(v) \sim \sum_{i=1}^{N} \operatorname{Exp}(i(n-i)),
$$

where $N$ is uniformly distributed over $1, \ldots, n-1$ and independent of the exponential distributions. Using this observation we can derive the following known results. 
Lemma 2.4. Let $D_{n}$ denote the distance between two arbitrarily chosen vertices in a random shortest path metric generated from $K_{n}$, the complete graph on $n$ vertices. Then

$$
\mathbb{E}\left[D_{n}\right]=\frac{H_{n-1}}{n-1}=\frac{\ln (n)}{n}+O\left(\frac{1}{n}\right),
$$

and

$$
\operatorname{Var}\left(D_{n}\right)=\frac{3 H_{n-1}^{(2)}}{n(n-1)}-\frac{H_{n-1}^{2}}{n(n-1)^{2}}=\frac{\pi^{2}}{2 n^{2}}-O\left(\frac{\ln ^{2}(n)}{n^{3}}\right)
$$

Proof. Using (2.1) and conditioning on the value of $N$, we can immediately see that

$$
\begin{aligned}
\mathbb{E}\left[D_{n}\right] & =\mathbb{E}\left[\sum_{i=1}^{N} \operatorname{Exp}(i(n-i))\right]=\sum_{k=1}^{n-1} \mathbb{E}\left[\sum_{i=1}^{k} \operatorname{Exp}(i(n-i))\right] \cdot \mathbb{P}(N=k) \\
& =\frac{1}{n-1} \cdot \sum_{k=1}^{n-1} \sum_{i=1}^{k} \mathbb{E}[\operatorname{Exp}(i(n-i))]=\frac{1}{n-1} \cdot \sum_{k=1}^{n-1} \sum_{i=1}^{k} \frac{1}{i(n-i)} \\
& =\frac{1}{n-1} \cdot H_{n-1}=\frac{\ln (n)}{n}+O\left(\frac{1}{n}\right),
\end{aligned}
$$

where the last equality follows from observing that $H_{n-1}=\ln (n)+\Theta(1)$.

In order to derive $\operatorname{Var}\left(D_{n}\right)$ we first compute the second moment, $\mathbb{E}\left[D_{n}^{2}\right]$. Conditioning on the value of $N$ and using the fact that we can write $\mathbb{E}\left[X^{2}\right]=$ $\operatorname{Var}(X)+(\mathbb{E}[X])^{2}$, we obtain

$$
\begin{aligned}
\mathbb{E}\left[D_{n}^{2}\right] & =\sum_{k=1}^{n-1} \mathbb{E}\left[\left(\sum_{i=1}^{k} \operatorname{Exp}(i(n-i))\right)^{2}\right] \cdot \mathbb{P}(N=k) \\
& =\frac{1}{n-1} \cdot \sum_{k=1}^{n-1}\left(\operatorname{Var}\left(\sum_{i=1}^{k} \operatorname{Exp}(i(n-i))\right)+\left(\mathbb{E}\left[\sum_{i=1}^{k} \operatorname{Exp}(i(n-i))\right]\right)^{2}\right) \\
& =\frac{1}{n-1} \cdot \sum_{k=1}^{n-1}\left(\sum_{i=1}^{k} \frac{1}{i^{2}(n-i)^{2}}+\left(\sum_{i=1}^{k} \frac{1}{i(n-i)}\right)^{2}\right) .
\end{aligned}
$$

Next, observe that

$$
\begin{aligned}
\sum_{k=1}^{n-1} \sum_{i=1}^{k} \frac{1}{i^{2}(n-i)^{2}} & =\sum_{i=1}^{n-1} \frac{1}{i^{2}(n-i)}=\sum_{i=1}^{n-1}\left(\frac{1}{i^{2} n}+\frac{1}{i n(n-i)}\right) \\
& =\frac{1}{n} \cdot \sum_{i=1}^{n-1} \frac{1}{i^{2}}+\frac{2 H_{n-1}}{n^{2}}
\end{aligned}
$$


and that

$$
\begin{aligned}
\sum_{k=1}^{n-1}\left(\sum_{i=1}^{k} \frac{1}{i(n-i)}\right)^{2} & =\sum_{k=1}^{n-1}\left(\frac{H_{n-1}+H_{k}-H_{n-k-1}}{n}\right)^{2} \\
& =\frac{(n+1) H_{n-1}^{2}}{n^{2}}+\sum_{k=1}^{n-1} \frac{\left(H_{k}-H_{n-k-1}\right)^{2}}{n^{2}} \\
& =\frac{(n+1) H_{n-1}^{2}}{n^{2}}+\frac{2}{n} \cdot \sum_{k=1}^{n-1} \frac{1}{k^{2}}-\frac{H_{n-1}^{2}+2 H_{n-1}}{n^{2}} \\
& =\frac{H_{n-1}^{2}}{n}+\frac{2}{n} \cdot \sum_{k=1}^{n-1} \frac{1}{k^{2}}-\frac{2 H_{n-1}}{n^{2}} .
\end{aligned}
$$

Combining these two results, we obtain

$$
\mathbb{E}\left[D_{n}^{2}\right]=\frac{H_{n-1}^{2}}{n(n-1)}+\frac{3}{n(n-1)} \cdot \sum_{k=1}^{n-1} \frac{1}{k^{2}} .
$$

Finally, using the fact that we can write $\operatorname{Var}(X)=\mathbb{E}\left[X^{2}\right]-(\mathbb{E}[X])^{2}$, we obtain

$$
\begin{aligned}
\operatorname{Var}\left(D_{n}\right) & =\mathbb{E}\left[D_{n}^{2}\right]-\left(\mathbb{E}\left[D_{n}\right]\right)^{2}=\frac{H_{n-1}^{2}}{n(n-1)}+\frac{3}{n(n-1)} \cdot \sum_{k=1}^{n-1} \frac{1}{k^{2}}-\frac{H_{n-1}^{2}}{(n-1)^{2}} \\
& =\frac{3 H_{n-1}^{(2)}}{n(n-1)}-\frac{H_{n-1}^{2}}{n(n-1)^{2}}=\frac{\pi^{2}}{2 n^{2}}-O\left(\frac{\ln ^{2}(n)}{n^{3}}\right)
\end{aligned}
$$

where the last equality follows from observing that $H_{n-1}=\ln (n)+\Theta(1)$ and $H_{n-1}^{(2)}=\pi^{2} / 6-\Theta(1 / n)$.

Additionally, we can use the idea behind (2.1) to derive the joint distribution of two distinct 'edges' that leave a vertex $u$. In particular, if $u, v, w \in V$ are three arbitrarily chosen distinct vertices, we can write

$$
D_{n} \sim \tau_{M+1}(u)=\sum_{i=1}^{M} Y_{i} \quad \text { and } \quad D_{n}^{\prime} \sim \tau_{N+1}(u)=\sum_{i=1}^{N} Y_{i}
$$

where $Y_{i} \sim \operatorname{Exp}(i(n-i))$ independently, and where $M$ and $N$ are uniformly distributed over $1, \ldots, n-1$, independent of the exponential distributions, but with the additional constraint $M \neq N$. In other words, $\mathbb{P}(M=j, N=k)=$ $1 /((n-1)(n-2))$ for all $j, k \in[n]$ with $j \neq k$ and $\mathbb{P}(M=j, N=k)=0$ otherwise. 
Using this joint distribution, we can derive an expression for the covariance $\operatorname{Cov}(d(u, v), d(u, w))$ between the distances of two adjacent 'edges' in a random shortest path metric generated from a complete graph. The following lemma provides the result. As expected, there is a positive correlation between the distances of two adjacent 'edges'.

Lemma 2.5. Let $u, v, w \in V$ be three arbitrarily chosen distinct vertices in a random shortest path metric generated from $K_{n}$, the complete graph on $n$ vertices, and let $D_{n}:=$ $d(u, v)$ and $D_{n}^{\prime}:=d(u, w)$. Then

$$
\begin{aligned}
\operatorname{Cov}\left(D_{n}, D_{n}^{\prime}\right) & =\left(1-\frac{3}{n}\right) \cdot \frac{H_{n-1}^{(2)}}{(n-1)(n-2)}+\frac{H_{n-1}^{2}}{n(n-1)^{2}(n-2)} \\
& =\frac{\pi^{2}}{6 n^{2}}-O\left(\frac{1}{n^{3}}\right) .
\end{aligned}
$$

Proof. Let $X_{k}:=\sum_{i=1}^{k} Y_{i}$ where $Y_{i} \sim \operatorname{Exp}(i(n-i))$ to shorten notation, and observe that

$$
\sum_{k=1}^{n-1} X_{k}=\sum_{k=1}^{n-1} \sum_{i=1}^{k} Y_{i}=\sum_{i=1}^{n-1}(n-i) Y_{i} \sim \sum_{i=1}^{n-1} \operatorname{Exp}(i),
$$

since $a \operatorname{Exp}(b) \sim \operatorname{Exp}(b / a)$ for all $a, b>0$. In particular, this implies that

$$
\operatorname{Var}\left(\sum_{k=1}^{n-1} X_{k}\right)=\operatorname{Var}\left(\sum_{i=1}^{n-1} \operatorname{Exp}(i)\right)=\sum_{i=1}^{n-1} \operatorname{Var}(\operatorname{Exp}(i))=\sum_{i=1}^{n-1} \frac{1}{i^{2}}=H_{n-1}^{(2)} .
$$

Since we can write $D_{n} \sim X_{M}$ and $D_{n}^{\prime} \sim X_{N}$, it follows using the law of total covariance that

$$
\begin{aligned}
\operatorname{Cov}\left(D_{n}, D_{n}^{\prime}\right) & =\operatorname{Cov}\left(X_{M}, X_{N}\right) \\
& =\mathbb{E}\left[\operatorname{Cov}\left(X_{M}, X_{N} \mid M, N\right)\right]+\operatorname{Cov}\left(\mathbb{E}\left[X_{M} \mid M, N\right], \mathbb{E}\left[X_{N} \mid M, N\right]\right) .
\end{aligned}
$$

Similarly, using the law of total variance, we obtain

$$
\operatorname{Var}\left(D_{n}\right)=\operatorname{Var}\left(X_{M}\right)=\mathbb{E}\left[\operatorname{Var}\left(X_{M} \mid M\right)\right]+\operatorname{Var}\left(\mathbb{E}\left[X_{M} \mid M\right]\right) .
$$

For reasons that become clear later in this proof we are interested in the value of $(n-1) \cdot \operatorname{Var}\left(\mathbb{E}\left[X_{M} \mid M\right]\right)+(n-1)(n-2) \cdot \operatorname{Cov}\left(\mathbb{E}\left[X_{M} \mid M, N\right], \mathbb{E}\left[X_{N} \mid M, N\right]\right)$. Define $E_{k}:=\mathbb{E}\left[X_{k}\right]=\sum_{i=1}^{k} 1 /(i(n-i))$ to shorten notation. Observe that $\mathbb{E}\left[X_{M} \mid M\right]$ is uniformly distributed over $E_{1}, \ldots, E_{n-1}$ and that the joint distribution of $Y_{M}:=\mathbb{E}\left[X_{M} \mid M, N\right]$ and $Y_{N}:=\mathbb{E}\left[X_{N} \mid M, N\right]$ satisfies $\mathbb{P}\left(Y_{M}=\right.$ $E_{j}$ and $\left.Y_{N}=E_{k}\right)=1 /(n-1)(n-2)$ for all $1 \leq j, k \leq n-1$ with $j \neq k$. Upon 
direct computation we now obtain

$$
\begin{gathered}
(n-1) \cdot \operatorname{Var}\left(\mathbb{E}\left[X_{M} \mid M\right]\right)+(n-1)(n-2) \cdot \operatorname{Cov}\left(\mathbb{E}\left[X_{M} \mid M, N\right], \mathbb{E}\left[X_{N} \mid M, N\right]\right) \\
=\frac{1}{2(n-1)} \cdot \sum_{j=1}^{n-1} \sum_{j^{\prime}=1}^{n-1}\left(E_{j}-E_{j^{\prime}}\right)^{2} \\
\quad+\frac{1}{2(n-1)(n-2)} \cdot \sum_{j=1}^{n-1} \sum_{\substack{k=1 \\
k \neq j}}^{n-1} \sum_{j^{\prime}=1}^{n-1} \sum_{\substack{k^{\prime}=1 \\
k^{\prime} \neq j^{\prime}}}^{n-1}\left(E_{j}-E_{j^{\prime}}\right)\left(E_{k}-E_{k^{\prime}}\right) \\
=\frac{1}{2(n-1)(n-2)} \cdot \sum_{j=1}^{n-1} \sum_{k=1}^{n-1} \sum_{j^{\prime}=1}^{n-1} \sum_{k^{\prime}=1}^{n-1}\left(E_{j}-E_{j^{\prime}}\right)\left(E_{k}-E_{k^{\prime}}\right)=0,
\end{gathered}
$$

where the last equality follows by utilising the symmetry. Combining this result with the equalities we obtained from the laws of total variance and covariance, it follows that

$$
\begin{aligned}
(n-1) & \cdot \operatorname{Var}\left(D_{n}\right)+(n-1)(n-2) \cdot \operatorname{Cov}\left(D_{n}, D_{n}^{\prime}\right) \\
& =(n-1) \cdot \mathbb{E}\left[\operatorname{Var}\left(X_{M} \mid M\right)\right]+(n-1)(n-2) \cdot \mathbb{E}\left[\operatorname{Cov}\left(X_{M}, X_{N} \mid M, N\right)\right] \\
& =\sum_{k=1}^{n-1} \operatorname{Var}\left(X_{k}\right)+\sum_{j=1}^{n-1} \sum_{\substack{k=1 \\
k \neq j}}^{n-1} \operatorname{Cov}\left(X_{j}, X_{k}\right)=\operatorname{Var}\left(\sum_{k=1}^{n-1} X_{k}\right)=H_{n-1}^{(2)},
\end{aligned}
$$

where the last equality follows from (2.2). Rearranging this equality and using the result of Lemma 2.4, we obtain

$$
\begin{aligned}
\operatorname{Cov}\left(D_{n}, D_{n}^{\prime}\right) & =\frac{H_{n-1}^{(2)}}{(n-1)(n-2)}-\frac{\operatorname{Var}\left(D_{n}\right)}{n-2} \\
& =\left(1-\frac{3}{n}\right) \cdot \frac{H_{n-1}^{(2)}}{(n-1)(n-2)}+\frac{H_{n-1}^{2}}{n(n-1)^{2}(n-2)} \\
& =\frac{\pi^{2}}{6 n^{2}}-O\left(\frac{1}{n^{3}}\right)
\end{aligned}
$$

where the last equality follows from observing that $H_{n-1}=\ln (n)+\Theta(1)$ and $H_{n-1}^{(2)}=\pi^{2} / 6-\Theta(1 / n)$.

\subsection{General Graphs}

In this section, we aim to generalize some known properties of random shortest path metrics generated from complete graphs to random shortest path metrics 
generated from general graphs. The properties in this chapter hold for arbitrary (families of) graphs. Other properties, tailored to more specific families of graphs, will be introduced in Chapters 3 and 4

We start off with some results regarding sums of lightest edge weights in a graph $G$. After that, we consider a random growth process which we use to derive stochastic bounds on the distribution of the distances in a random shortest path metric, in particular on the distribution of $\tau_{k}(v)$. This random growth process is a generalization of the random growth process from the previous section. Finally, we provide a conditional clustering result which is a generalization of the clustering result for random shortest path metrics generated from complete graphs by Bringmann et al. [15].

\subsubsection{Sums of Lightest Edge Weights in $G$}

The objective of many combinatorial optimization problems is to minimize a sum of distances, with some constraints. For the probabilistic analyses of such problems, it is useful to have lower bounds for the optimal solutions. It turns out that we can use the value of sums of lightest edge weights in $G$ as a lower bound for the optimal solution of the combinatorial optimization problems that we introduced in Chapter 1 . In this subsection we derive some helpful results concerning these sums. Note that, in essence, these results merely are some advanced properties of the exponential distribution.

We start by probabilistically bounding the distribution of these sums of lightest edge weights in $G$ between two Erlang distributions.

Lemma 2.6. Let $S_{m}$ denote the sum of the $m$ lightest edge weights in $G$. Then

$$
\sum_{i=0}^{m-1} \operatorname{Exp}\left(\frac{e|E|}{m}\right) \precsim S_{m} \precsim \sum_{i=0}^{m-1} \operatorname{Exp}\left(\frac{|E|}{m}\right) .
$$

Proof. Let $\sigma_{k}$ denote the $k$ th lightest edge weight in $G$. Since all edge weights are independent and standard exponentially distributed, we have $\sigma_{1}=S_{1} \sim$ $\operatorname{Exp}(|E|)$. Using the memorylessness property of the exponential distribution, it follows that $\sigma_{2} \sim \sigma_{1}+\operatorname{Exp}(|E|-1)$, i.e., the second lightest edge weight is equal to the lightest edge weight plus the minimum of $|E|-1$ standard exponential distributed random variables. In general, we get $\sigma_{k+1} \sim \sigma_{k}+\operatorname{Exp}(|E|-k)$. The definition $S_{m}=\sum_{k=1}^{m} \sigma_{k}$ yields

$$
S_{m} \sim \sum_{i=1}^{m-1}(m-i) \cdot \operatorname{Exp}(|E|-i) \sim \sum_{i=0}^{m-1} \operatorname{Exp}\left(\frac{|E|-i}{m-i}\right) .
$$

Now, the first stochastic dominance relation follows from Lemma 2.3 by observing that

$$
\prod_{i=0}^{m-1} \frac{|E|-i}{m-i}=\frac{|E| !}{m !(|E|-m) !}=\left(\begin{array}{c}
|E| \\
m
\end{array}\right) \leq\left(\frac{e|E|}{m}\right)^{m},
$$


where the inequality follows from applying the well-known inequality $\left(\begin{array}{l}n \\ k\end{array}\right) \leq$ $(e n / k)^{k}$.

The second stochastic dominance relation follows by observing that $|E| \geq m$, which implies that $(|E|-i) /(m-i) \geq|E| / m$ for all $i=0, \ldots, m-1$.

Using the stochastic dominance relations in the foregoing lemma, we can easily derive the expected values of the sums of the lightest edge weights in $G$ up to a constant factor.

Corollary 2.7. Let $S_{m}$ denote the sum of the $m$ lightest edge weights in $G$. Then $\mathbb{E}\left[S_{m}\right]=\Theta\left(m^{2} /|E|\right)$.

Proof. From Lemma 2.6 we can immediately see that

$$
\mathbb{E}\left[\sum_{i=0}^{m-1} \operatorname{Exp}\left(\frac{e|E|}{m}\right)\right] \leq \mathbb{E}\left[S_{m}\right] \leq \mathbb{E}\left[\sum_{i=0}^{m-1} \operatorname{Exp}\left(\frac{|E|}{m}\right)\right] .
$$

The result follows by observing that

$$
\mathbb{E}\left[\sum_{i=0}^{m-1} \operatorname{Exp}\left(\frac{e|E|}{m}\right)\right]=\frac{m^{2}}{e|E|} \quad \text { and } \quad \mathbb{E}\left[\sum_{i=0}^{m-1} \operatorname{Exp}\left(\frac{|E|}{m}\right)\right]=\frac{m^{2}}{|E|} .
$$

We can also use the result of Lemma 2.6 to derive the following upper bound on the cumulative distribution function of the sum of the lightest edge weights in $G$.

Lemma 2.8. Let $S_{m}$ denote the sum of the $m$ lightest edge weights in $G$. Then, for any $s>0$ we have

$$
\mathbb{P}\left(S_{m} \leq s\right) \leq \exp \left(m\left(2+\ln \left(\frac{|E| s}{m^{2}}\right)\right)\right)=\left(\frac{e^{2}|E| s}{m^{2}}\right)^{m} .
$$

Proof. First of all, Lemma 2.6yields

$$
S_{m} \succsim \sum_{i=0}^{m-1} \operatorname{Exp}\left(\frac{e|E|}{m}\right) .
$$

Now, we apply Corollary 2.2 with $\mu=m^{2} / e|E|, a_{*}=e|E| / m$, and $x=s$ to obtain

$$
\mathbb{P}\left(S_{m} \leq s\right) \leq \mathbb{P}\left(\sum_{i=0}^{m-1} \operatorname{Exp}\left(\frac{e|E|}{m}\right) \leq s\right) \leq \exp \left(m\left(1+\ln \left(\frac{e|E| s}{m^{2}}\right)\right)\right) .
$$

The result follows immediately. 


\subsubsection{A Random Growth Process}

In order to understand the structure of random shortest path metrics it is important to get a feeling for the distribution of the distances in the random metric, in particular the distribution of $\tau_{k}(v)$. In this subsection, we use a generalization of the random growth process that (among others) has been used by Davis and Prieditis [20], Janson [33], and Bringmann et al. [15] to analyse the model of random shortest path metrics generated from complete graphs. Using the symmetry of complete graphs, they were able to derive the exact distribution of $\tau_{k}(v)$ and they used this distribution to infer some properties of the model. For random shortest path metrics generated from non-complete graphs, the distribution of $\tau_{k}(v)$ heavily depends on the exact position of $v$ within the graph $G$ (and also on the graph $G$ itself).

In general, we do not have sufficient information to find an exact distribution of $\tau_{k}(v)$ in this case. Instead we derive stochastic upper and lower bounds on $\tau_{k}(v)$ which depend roughly on the connectivity of the graph $G$, and which hold for any vertex $v \in V$. The following definition introduces this property. In this definition, for any $U \subseteq V, G[U]$ denotes the subgraph of $G=(V, E)$ induced by $U$, i.e., the subgraph of $G$ whose vertex set is $U$ and whose edge set consists of all edges in $E$ with both endpoints in $U$, and $\delta_{G}(U)$ denotes the cut (in $G$ ) induced by $U$, i.e., $\delta_{G}(U):=\{\{u, v\} \in E \mid u \in U, v \notin U\}$.

Definition 2.9. Let $G=(V, E)$ be a finite simple connected graph on $n$ vertices. For each $k \in[n]$, let $\mathcal{P}_{k}^{c}(G)$ denote the set of all subsets $U \subseteq V$ with $|U|=k$ such that $G[U]$ is a connected subgraph of $G$. Then we define the cut parameters of $G$ by

$$
\alpha_{k}^{G}:=\min _{U \in \mathcal{P}_{k}^{c}(G)}\left|\delta_{G}(U)\right| \quad \text { and } \quad \beta_{k}^{G}:=\max _{U \in \mathcal{P}_{k}^{c}(G)}\left|\delta_{G}(U)\right| .
$$

Observe that $\alpha_{k}^{G}=\beta_{k}^{G}=k(n-k)$ for any $k \in[n]$ if $G$ is a complete graph on $n$ vertices. Since the analysis of the random growth process that has been used to analyse random shortest path metrics generated from complete graphs heavily relies on the fact that $\left|\delta_{G}(U)\right|=|U| \cdot(|V|-|U|)$ for any $U \subseteq V$ when $G$ is the complete graph, we can use this definition to generalize the random growth process. The (proof of) the following lemma shows this generalization.

Lemma 2.10. Let $G=(V, E)$ be a finite simple connected graph on $n$ vertices. For any $v \in V$ and any $k \in[n]$ we have

$$
\sum_{i=1}^{k-1} \operatorname{Exp}\left(\beta_{i}^{G}\right) \precsim \tau_{k}(v) \precsim \sum_{i=1}^{k-1} \operatorname{Exp}\left(\alpha_{i}^{G}\right) .
$$

Proof. The values of $\tau_{k}(v)$ are generated by a birth process as follows. For $k=1$ we have $\tau_{k}(v)=0$ and also $\sum_{i=1}^{k-1} \operatorname{Exp}\left(\alpha_{i}^{G}\right)=\sum_{i=1}^{k-1} \operatorname{Exp}\left(\beta_{i}^{G}\right)=0$. For $k \geq 2$ we can obtain $\tau_{k}(v)$ from $\tau_{k-1}(v)$ by looking at all edges that 'leave' 
$B_{\tau_{k-1}}(v)$, i.e., edges $(u, x)$ with $u \in B_{\tau_{k-1}}(v)$ and $x \notin B_{\tau_{k-1}}(v)$. By definition there are $\chi_{k-1}(v)$ such edges, and using the notation introduced in Definition 2.9 it follows that $\alpha_{k-1}^{G} \leq \chi_{k-1}(v) \leq \beta_{k-1}^{G}$. Moreover, by definition of $\tau_{k-1}(v)$, these edges are conditioned to have a weight of at least $\tau_{k-1}(v)-$ $d(v, u)$ (otherwise we would have $d(v, x) \leq d(v, u)+d(u, x)<\tau_{k-1}(v)$ ). Using the memorylessness of the exponential distribution, it follows that $\tau_{k}(v)-$ $\tau_{k-1}(v)$ is the minimum of $\chi_{k-1}(v)$ exponential random variables (with parameter 1), or, equivalently $\tau_{k}(v)-\tau_{k-1}(v) \sim \operatorname{Exp}\left(\chi_{k-1}(v)\right)$. We also know that $\operatorname{Exp}\left(\beta_{k-1}^{G}\right) \precsim \operatorname{Exp}\left(\chi_{k-1}(v)\right) \precsim \operatorname{Exp}\left(\alpha_{k-1}^{G}\right)$ since $\alpha_{k-1}^{G} \leq \chi_{k-1}(v) \leq \beta_{k-1}^{G}$. So, we obtain $\operatorname{Exp}\left(\beta_{k-1}^{G}\right) \precsim \tau_{k}(v)-\tau_{k-1}(v) \precsim \operatorname{Exp}\left(\alpha_{k-1}^{G}\right)$. The result follows using induction.

The tightness of the stochastic approximation in this lemma depends heavily on the graph $G$. In the following chapters we will consider two families of graphs for which this approximation is sufficiently tight in order to obtain interesting results: (dense) Erdős-Rényi random graphs in Chapter 3 and sparse graphs in Chapter 4

We conclude this subsection by stating the following corollary which follows immediately from the previous lemma.

Corollary 2.11. Let $G=(V, E)$ be a finite simple connected graph on $n$ vertices. For any $v \in V$ and any $k \in[n]$ we have

$$
\sum_{i=1}^{k-1} \frac{1}{\beta_{i}^{G}} \leq \mathbb{E}\left[\tau_{k}(v)\right] \leq \sum_{i=1}^{k-1} \frac{1}{\alpha_{i}^{G}} .
$$

\subsubsection{Clustering}

The last property of random shortest path metrics that we consider in this chapter is the so-called clustering of the vertices. Bringmann et al. [15] have shown that, for any given diameter, the number of clusters needed to partition instances of random shortest path metrics generated from complete graphs into clusters of at most that diameter is small. This observation was the main ingredient for their probabilistic analysis of some greedy-like heuristics for the minimumdistance perfect matching problem and the traveling salesman problem.

In this thesis we will make use of two similar results that show that also instances of random shortest path metrics generated from certain non-complete graphs can be clustered into a sufficiently small number of clusters, for any given diameter. As we will see in Chapters 3 and 4 , these clustering results enable us to show that greedy choices of heuristics for the minimum-distance perfect matching problem and the traveling salesman problem are unlikely to be 'really bad'.

The following theorem provides the general form of these clustering results. Its proof follows closely the ideas of Bringmann et al. [15], albeit with a different value of $s_{\Delta}$. The condition of the theorem might seem rather restrictive at first, 
but we show that it is satisfied by (dense) Erdős-Rényi random graphs (Chapter 3) and (a large class of) sparse graphs (Chapter 4 .

Theorem 2.12. Let $\mathcal{G}$ be a family of finite simple connected graphs, and suppose that there exist constants $c_{1}, c_{2}$ and a function $f(\Delta, n)$ such that

$$
\mathbb{P}\left(\left|B_{\Delta}(v)\right|<\min \left\{f(\Delta, n), c_{2} n\right\}\right) \leq \frac{c_{1}}{f(\Delta, n)}
$$

for any $\Delta>0$, any $v \in V$ and any $G=(V, E) \in \mathcal{G}$ with $n$ sufficiently large. Then, for any $\Delta>0$ and any $G \in \mathcal{G}$ there exists a partition of the vertices of a random shortest path metric generated from $G$ into clusters, each of diameter at most $4 \Delta$, such that the expected number of clusters needed is bounded from above by

$$
\frac{1}{c_{2}}+\frac{\left(c_{1}+1\right) n}{f(\Delta, n)} .
$$

Proof. Let $G \in \mathcal{G}$ with $n$ sufficiently large, and let $s_{\Delta}:=\min \left\{f(\Delta, n), c_{2} n\right\}$. Consider a random shortest path metric generated from $G$. We call vertex $v \Delta$-dense if $\left|B_{\Delta}(v)\right| \geq s_{\Delta}$ and $\Delta$-sparse otherwise. Using the conditions of this theorem we can bound the expected number of $\Delta$-sparse vertices by $c_{1} n / f(\Delta, n)$. We put each $\Delta$-sparse vertex in its own cluster (of size 1 ), which has diameter $0 \leq 4 \Delta$. Now, only the $\Delta$-dense vertices remain. We cluster them according to the following process. Consider an auxiliary graph $H$ whose vertices are the $\Delta$-dense vertices and where two vertices $u, v$ are connected by an edge if and only if $B_{\Delta}(u) \cap B_{\Delta}(v) \neq \varnothing$. Consider an arbitrary maximal independent set $S$ in $H$, and observe that $|S| \leq n / s_{\Delta}$ by construction of $H$. We create initial clusters $C_{1}, \ldots, C_{|S|}$, each of which equals $B_{\Delta}(v)$ for some vertex $v \in S$. Observe that these initial clusters have diameter at most $2 \Delta$.

Next, consider an arbitrary $\Delta$-dense vertex $v$ that is not yet part of any cluster. By the maximality of $S$, we know that there must exist a vertex $u \in S$ such that $A:=B_{\Delta}(u) \cap B_{\Delta}(v) \neq \varnothing$. Let $x \in A$ be arbitrarily chosen, and observe that $d(v, u) \leq d(v, x)+d(x, u) \leq \Delta+\Delta=2 \Delta$. We add $v$ to the initial cluster corresponding to $u$, and repeat this step until all $\Delta$-dense vertices have been added to some initial cluster. By construction, the diameter of all these clusters is now at most $4 \Delta$ : consider two arbitrary vertices $w, y$ in a cluster that initially corresponded to $u \in S$; then we have $d(w, y) \leq d(w, u)+d(u, y) \leq 2 \Delta+2 \Delta=4 \Delta$. So, now we have in expectation at most $c_{1} n / f(\Delta, n)$ clusters containing one $(\Delta$ sparse) vertex each, and at most $n / s_{\Delta} \leq 1 / c_{2}+n / f(\Delta, n)$ clusters containing at least $s_{\Delta}(\Delta$-dense) vertices each, all with diameter at most $4 \Delta$. The result follows.

Remark. Note that we can obtain the 'original' clustering result from Bringmann et al. [15] for random shortest path metrics generated from complete graphs by using $c_{1}=1, c_{2}=1 / 2$ and $f(\Delta, n)=\exp (\Delta n / 5)$. 


\section{Chapter 3}

\section{Analysis of Heuristics on RSPM from Dense ERRG Graphs}

Our first step in the direction of conducting probabilistic analyses for optimization problems on random shortest path metrics generated from non-complete graphs is the usage of (dense) Erdős-Rényi random graphs. In this chapter we present our results for this model.

Our first approach utilizes the fact that the cut parameters introduced in the previous chapter can be bounded tightly with high probability for dense Erdős-Rényi random graphs. Inspired by this observation, we provide some structural properties of random shortest path metrics generated from arbitrary graphs based on these cut parameters, including an application of the general clustering result from Chapter 2 These properties can be seen as a generalization of some of the structural properties found by Bringmann et al. [15].

Next, we use these structural insights to perform a probabilistic analysis for some simple heuristics for several combinatorial optimization problems, where the results still depend on the cut parameters. Finally, we use the tight bounds on the cut parameters of Erdős-Rényi random graphs to obtain the main results from this chapter. This first approach is based on [42, 43].

We end this chapter with a section dedicated to a different approach that can be used to obtain (a slightly weaker version of) these main results. This second approach uses a coupling argument that shows that (with high probability) random shortest path metrics generated from (dense) Erdős-Rényi random graphs are a scaled version of random shortest path metrics generated from complete graphs. 


\subsection{Erdős-Rényi Random Graphs}

In this chapter we consider random shortest path metrics generated from (dense) Erdős-Rényi random graphs. An undirected graph $G=(V, E)$ generated by the $G(n, p)$ model has $n$ vertices $(V=[n])$ and between each pair of vertices an edge is included with probability $p$, independent of every other pair.

Working with Erdős-Rényi random graphs introduces an extra amount of stochasticity to the probabilistic analysis, since both the graph and the edge weights are random. This extra stochasticity makes the analysis significantly more complicated. So, in order to avoid this extra stochasticity as long as possible, in Sections 3.2 and 3.3 we start our analysis using an arbitrary fixed (deterministic) graph G. Later on, from Section 3.4 onwards we consider Erdős-Rényi random graphs again.

In our probabilistic analyses, we heavily use the fact that the cut parameters of a (dense) Erdős-Rényi random graph can be bounded tightly with high probability. The well-known result in the following lemma makes this idea precise. We include its proof for completeness.

Lemma 3.1. Let $\varepsilon \in(0,1)$ and $c>9 / \varepsilon^{2}$ be constant. Let $G=(V, E)$ be an instance of the $G(n, p)$ model for $p \geq c \ln (n) / n$ (as $n \rightarrow \infty)$. Then the cut parameters of $G$ are bounded by $(1-\varepsilon) p k(n-k) \leq \alpha_{k}^{G} \leq \beta_{k}^{G} \leq(1+\varepsilon) p k(n-k)$ for all $k \in[n]$ with probability at least $1-o\left(1 / n^{2}\right)$.

Proof. Let $\mathcal{E}$ denote the event that the cut parameters of $G$ are not bounded by $(1-\varepsilon) p k(n-k) \leq \alpha_{k}^{G} \leq \beta_{k}^{G} \leq(1+\varepsilon) p k(n-k)$ for all $k \in[n]$. Using the definition of the cut parameters, the probability of this event can be written as

$$
\mathbb{P}(\mathcal{E})=\mathbb{P}\left(\exists k \in[n / 2] \exists U \in \mathcal{P}_{k}^{c}(G):|| \delta_{G}(U)|-p k(n-k)|>\varepsilon p k(n-k)\right) .
$$

Note that we can restrict ourselves here to connected subsets $U$ of size at most $n / 2$ since $U$ and $V \backslash U$ induce the same cut of $G$. Using the union bound and observing that $\left|\mathcal{P}_{k}^{c}(G)\right| \leq\left(\begin{array}{l}n \\ k\end{array}\right)$, we can bound this probability by

$$
\mathbb{P}(\mathcal{E}) \leq \sum_{k=1}^{n / 2}\left(\begin{array}{l}
n \\
k
\end{array}\right) \cdot \mathbb{P}\left(|| \delta_{G}\left(U_{k}\right)|-p k(n-k)|>\varepsilon p k(n-k)\right),
$$

where $U_{k}$ is an arbitrary subset of $V$ of size $k$. Applying a Chernoff bound [51. Cor. 4.6] to each term of this summation, we can further bound this by

$$
\mathbb{P}(\mathcal{E}) \leq \sum_{k=1}^{n / 2}\left(\begin{array}{l}
n \\
k
\end{array}\right) 2 e^{-\varepsilon^{2} p k(n-k) / 3} \leq \sum_{k=1}^{n / 2}\left(\begin{array}{l}
n \\
k
\end{array}\right) 2 e^{-\varepsilon^{2} c \ln (n) k(n-k) / 3 n},
$$

where we used $p \geq c \ln (n) / n$ for the last inequality. Now, let $\xi>0$ be a sufficiently small constant $\left(\xi<1-9 / c \varepsilon^{2}\right.$ satisfies this). Using this $\xi$ we split the 
summation in two parts, and then use the bounds $\left(\begin{array}{l}n \\ k\end{array}\right) \leq n^{k}$ and $\left(\begin{array}{l}n \\ k\end{array}\right) \leq 2^{n}$, respectively, to obtain

$$
\begin{aligned}
\mathbb{P}(\mathcal{E}) & \leq \sum_{k=1}^{\xi n}\left(\begin{array}{l}
n \\
k
\end{array}\right) 2 e^{-\varepsilon^{2} c \ln (n) k(n-k) / 3 n}+\sum_{k=\xi \xi}^{n / 2}\left(\begin{array}{l}
n \\
k
\end{array}\right) 2 e^{-\varepsilon^{2} c \ln (n) k(n-k) / 3 n} \\
& \leq \sum_{k=1}^{\xi n} 2 e^{k \ln (n)\left(1-\varepsilon^{2} c(1-k / n) / 3\right)}+\sum_{k=\xi}^{n / 2} 2 e^{n \ln (2)-\varepsilon^{2} c \ln (n) k(n-k) / 3 n} \\
& \leq \sum_{k=1}^{\xi n} 2 e^{k \ln (n)\left(1-\varepsilon^{2} c(1-\xi) / 3\right)}+\sum_{k=\xi^{\eta} n}^{n / 2} 2 e^{n \ln (2)-\varepsilon^{2} c \ln (n) \xi(1-\xi) n / 3} .
\end{aligned}
$$

For the last inequality we used the facts that $k / n \leq \xi$ for all $1 \leq k \leq \xi n$ and $k(n-k) \geq \xi(1-\xi) n^{2}$ for all $\xi n \leq k \leq n / 2$. Now, since $\xi$ is sufficiently small, we have $1-\varepsilon^{2} c(1-\xi) / 3<-2$ and thus we can bound the first summation by $o\left(1 / n^{2}\right)$. Furthermore, as $n \rightarrow \infty$, each summand of the second summation is bounded by $e^{-\Omega(n \ln (n))}=n^{-\Omega(n)}$, which allows us to bound the second summation by $n \cdot n^{-\Omega(n)}=n^{-\Omega(n)}$. Together with the bound for the first summation this yields $\mathbb{P}(\mathcal{E}) \leq o\left(1 / n^{2}\right)+n^{-\Omega(n)}=o\left(1 / n^{2}\right)$. The result now follows by taking the complement of the event $\mathcal{E}$.

\subsection{Structural Properties}

In this section we provide some structural properties regarding random shortest path metrics generated from an arbitrary fixed undirected connected simple graph $G$ (on $n$ vertices). These properties are used later on in our probabilistic analyses of the greedy-like heuristics for the minimum-distance perfect matching problem, the traveling salesman problem and the $k$-median problem in such random spaces. The results in this section can be seen as generalizations of the results by Bringmann et al. for random shortest path metrics generated from complete graphs [15] to the case of random shortest path metrics generated from an arbitrary graph G. Although this generalization might seem straightforward at first sight, it brings up some new difficulties that need to be addressed. Most notably, since we do not restrict ourselves to the complete graph, we cannot make use anymore of its symmetry and regularity.

We partially solve this problem by using the cut parameters that we introduced in Chapter 2. Moreover, in order to be able to utilize the result from Lemma 3.1 later on, we define the parameters $\alpha^{G}$ and $\beta^{G}$ as

$$
\alpha^{G}:=\min _{k \in[n-1]} \frac{\alpha_{k}^{G}}{k(n-k)} \quad \text { and } \quad \beta^{G}:=\max _{k \in[n-1]} \frac{\beta_{k}^{G}}{k(n-k)} .
$$


Using these definitions, the cut parameters of $G$ are bounded by $\alpha^{G} k(n-k) \leq$ $\alpha_{k}^{G} \leq \beta_{k}^{G} \leq \beta^{G} k(n-k)$ for all $k \in[n]$. It follows immediately that $0<\alpha^{G} \leq$ $\beta^{G} \leq 1$ for any finite simple connected graph $G$. We also observe that for a complete graph $\left(G=K_{n}\right)$ we have $\alpha^{G}=\beta^{G}=1$. The results in this section will be the most meaningful for graphs $G$ for which the parameters $\alpha^{G}$ and $\beta^{G}$ are relatively 'close' to each other.

We start by applying the general results concerning a random growth process in Section 2.3.2 to the case where we have bounds as introduced in (3.1). Then we use this result to derive a tail bound for the diameter of the random metric space, and to derive a specific bound on the distribution of the 'ball-sizes' in random shortest shortest metrics generated from a fixed graph $G$, which depends on the parameter $\alpha^{G}$. The latter bound is then being used to be able to apply the clustering result from Section 2.3.3.

\subsubsection{A Random Growth Process}

As mentioned in Chapter 2, in order to understand the structure of random shortest path metrics it is important to get a feeling for the distribution of the distances in the random metric, in particular the distribution of $\tau_{k}(v)$. The following corollary provides a stochastic bound for the distribution of $\tau_{k}(v)$. It is an immediate consequence of Lemma 2.10 and the definition of $\alpha^{G}$ and $\beta^{G}$.

Corollary 3.2. Let $G=(V, E)$ be a finite simple connected graph on $n$ vertices. For any $v \in V$ and any $k \in[n]$ we have

$$
\sum_{i=1}^{k-1} \operatorname{Exp}\left(\beta^{G} i(n-i)\right) \precsim \tau_{k}(v) \precsim \sum_{i=1}^{k-1} \operatorname{Exp}\left(\alpha^{G} i(n-i)\right) .
$$

Exploiting the linearity of expectation, the fact that the expected value of an exponentially distributed random variable with parameter $\lambda$ is $1 / \lambda$ and the fact that $\sum_{i=1}^{k-1} 1 /(i(n-i))=\left(H_{k-1}+H_{n-1}-H_{n-k}\right) / n$, we obtain the following corollary.

Corollary 3.3. Let $G=(V, E)$ be a finite simple connected graph on $n$ vertices. For any $v \in V$ and any $k \in[n]$ we have

$$
\frac{H_{k-1}+H_{n-1}-H_{n-k}}{\beta^{G} n} \leq \mathbb{E}\left[\tau_{k}(v)\right] \leq \frac{H_{k-1}+H_{n-1}-H_{n-k}}{\alpha^{G} n} .
$$

From this result we can derive the following extensions of two known results. First of all, if we randomly pick two vertices $u, v \in V$, then averaging the result of Corollary 3.3 over $k$ yields that the expected distance between them, $\mathbb{E}[d(u, v)]=\frac{1}{n-1} \sum_{k=2}^{n} \mathbb{E}\left[\tau_{k}(v)\right]$, is bounded between $H_{n-1} /\left(\beta^{G}(n-1)\right) \approx$ $\ln (n) / \beta^{G} n$ and $H_{n-1} /\left(\alpha^{G}(n-1)\right) \approx \ln (n) / \alpha^{G} n$, which is in line with the corresponding known result for complete graphs, where we have $\mathbb{E}[d(u, v)] \approx$ 
$\ln (n) / n[15,20,33]$. Secondly, for any vertex $v$, the longest distance from it to another vertex is $\tau_{n}(v)$. Its expectation is bounded between $2 H_{n-1} /\left(\beta^{G}(n-1)\right) \approx$ $2 \ln (n) / \beta^{G} n$ and $2 H_{n-1} /\left(\alpha^{G}(n-1)\right) \approx 2 \ln (n) / \alpha^{G} n$, which also is in line with the known result for complete graphs, where we have an expected value of approximately $2 \ln (n) / n[15,33$.

Using the result of Lemma 3.1. we can see that, for the case of the application to the Erdős-Rényi random graph model, with high probability over the random graph, $\mathbb{E}[d(u, v)]$ is approximately bounded between $\ln (n) /((1+\varepsilon) n p)$ and $\ln (n) /((1-\varepsilon) n p)$ for any constant $\varepsilon \in(0,1)$. This is in line with the known result $\mathbb{E}[d(u, v)] \approx \ln (n) / n p$ for $p$ sufficiently large [10].

It is also possible to find bounds for the cumulative distribution function of $\tau_{k}(v)$. To do so, we define $F_{k}(x):=\mathbb{P}\left(\tau_{k}(v) \leq x\right)$ for some arbitrary fixed vertex $v \in V$. We start by stating the following lemma, which provides an exact cumulative distribution function for a specific generalized Erlang distribution.

Lemma 3.4 ([15, Lemma 3.2]). Let $X \sim \sum_{i=1}^{n} \operatorname{Exp}(c i)$. Then, for any $a \geq 0$ we have $\mathbb{P}(X \leq a)=\left(1-e^{-c a}\right)^{n}$.

Using this cumulative distribution function, we can now derive a first upper and lower bound for $F_{k}(x)$.

Lemma 3.5. Let $G=(V, E)$ be a finite simple connected graph on $n$ vertices. For any $x \geq 0$ and any $k \in[n]$ we have

$$
\left(1-\exp \left(-\alpha^{G}(n-k) x\right)\right)^{k-1} \leq F_{k}(x) \leq\left(1-\exp \left(-\beta^{G} n x\right)\right)^{k-1} .
$$

Proof. From Corollary 3.2 we know that

$$
\sum_{i=1}^{k-1} \operatorname{Exp}\left(\beta^{G} i(n-i)\right) \precsim \tau_{k}(v) \precsim \sum_{i=1}^{k-1} \operatorname{Exp}\left(\alpha^{G} i(n-i)\right) .
$$

Since $n i \geq i(n-i) \geq(n-k) i$ for all $i \in[k-1]$, it follows that $\operatorname{Exp}\left(\beta^{G} n i\right) \precsim$ $\operatorname{Exp}\left(\beta^{G} i(n-i)\right)$ and $\operatorname{Exp}\left(\alpha^{G} i(n-i)\right) \precsim \operatorname{Exp}\left(\alpha^{G}(n-k) i\right)$ for all $i \in[k-1]$. From this we obtain

$$
\sum_{i=1}^{k-1} \operatorname{Exp}\left(\beta^{G} n i\right) \precsim \tau_{k}(v) \precsim \sum_{i=1}^{k-1} \operatorname{Exp}\left(\alpha^{G}(n-k) i\right) .
$$

Combining this with the definition of stochastic dominance and with the result of Lemma 3.4, we obtain the desired result.

We can improve the lower bound for $F_{k}(x)$ slightly, as the following lemma shows. 
Lemma 3.6. Let $G=(V, E)$ be a finite simple connected graph on $n$ vertices. For any $x \geq 0$ and any $k \in[n]$ we have

$$
F_{k}(x) \geq\left(1-\exp \left(-\alpha^{G} n x / 4\right)\right)^{n}
$$

Proof. Note that $\tau_{k}(v)$ is monotonically increasing in $k$. This implies $F_{k+1}(x) \leq$ $F_{k}(x)$, so we only need to prove our claim for the case $k=n$. In this case, by Corollary 3.2, we have $\tau_{n}(v) \precsim \sum_{i=1}^{n-1} \operatorname{Exp}\left(\lambda_{i}\right)$ with $\lambda_{i}:=\alpha \alpha_{i}(n-i)=\lambda_{n-i}$. Exploiting the symmetry around $n / 2$, we obtain

$$
\tau_{n}(v) \precsim \sum_{i=1}^{n / 2} \operatorname{Exp}\left(\lambda_{i}\right)+\sum_{i=1}^{n / 2} \operatorname{Exp}\left(\lambda_{i}\right)
$$

This enables us to find a lower bound for $F_{n}(x)$ as follows:

$$
\begin{aligned}
F_{n}(x)=\mathbb{P}\left(\tau_{n}(v) \leq x\right) & \geq \mathbb{P}\left(\sum_{i=1}^{n / 2} \operatorname{Exp}\left(\lambda_{i}\right)+\sum_{i=1}^{n / 2} \operatorname{Exp}\left(\lambda_{i}\right) \leq x\right) \\
& \geq \mathbb{P}\left(\sum_{i=1}^{n / 2} \operatorname{Exp}\left(\lambda_{i}\right) \leq x / 2\right)^{2} .
\end{aligned}
$$

Since $i(n-i) \geq i n / 2$ for all $i \in[n / 2]$, we have $\operatorname{Exp}\left(\lambda_{i}\right) \precsim \operatorname{Exp}\left(\alpha^{G} i n / 2\right)$. Combining this with Lemma 3.4 yields

$$
F_{n}(x) \geq \mathbb{P}\left(\sum_{i=1}^{n / 2} \operatorname{Exp}\left(\alpha^{G} i n / 2\right) \leq x / 2\right)^{2}=\left(1-\exp \left(-\alpha^{G} n x / 4\right)\right)^{2\lfloor n / 2\rfloor}
$$

Using the inequality $2\lfloor n / 2\rfloor \leq n$ we end up with the desired result.

Using this improved lower bound for the cumulative distribution function of $\tau_{k}(v)$, we can derive the following tail bound for the diameter of the random metric space: $\Delta_{\max }=\max _{u, v} d(u, v) \leq O\left(\log (n) / \alpha^{G} n\right)$ with high probability.

Lemma 3.7. Let $G=(V, E)$ be a finite simple connected graph on $n$ vertices. For any fixed constant $c$ we have

$$
\mathbb{P}\left(\Delta_{\max }>\frac{c \ln (n)}{\alpha^{G} n}\right) \leq n^{2-c / 4}
$$

Proof. Clearly, we have $\Delta_{\max }=\max _{v} \tau_{n}(v)$. For $v \in V$, let $\mathcal{E}_{v}$ denote the event that $\tau_{n}(v)>c \ln (n) / \alpha^{G} n$. From Lemma 3.6 we know that $\mathbb{P}\left(\mathcal{E}_{v}\right)=$ $1-F_{n}\left(c \ln (n) / \alpha^{G} n\right) \leq 1-(1-\exp (-c \ln (n) / 4))^{n}$. Combining this with a 
union bound, we can derive that

$$
\mathbb{P}\left(\Delta_{\max }>\frac{c \ln (n)}{\alpha^{G} n}\right) \leq \sum_{v \in V} \mathbb{P}\left(\mathcal{E}_{v}\right) \leq n \cdot\left(1-\left(1-n^{-c / 4}\right)^{n}\right) \leq n^{2-c / 4},
$$

where the last inequality follows from Bernoulli's inequality.

\subsubsection{Clustering}

In this section we use our first bound on the cumulative distribution function of $\tau_{k}(v)$ to derive a bound that enables us to use the powerful clustering result from Section 2.3.3.

Lemma 3.8. Let $G=(V, E)$ be a finite simple connected graph on $n \geq 5$ vertices. For any fixed $\Delta \geq 0$ we have

$$
\mathbb{P}\left(\left|B_{\Delta}(v)\right|<\min \left\{\exp \left(\alpha^{G} \Delta n / 5\right), \frac{n+1}{2}\right\}\right) \leq \exp \left(-\alpha^{G} \Delta n / 5\right) .
$$

Proof. We have $\left|B_{\Delta}(v)\right| \geq k$ if and only if $\tau_{k}(v) \leq \Delta$. Using Lemma 3.5. we obtain

$$
\begin{aligned}
\mathbb{P}\left(\left|B_{\Delta}(v)\right|<\min \right. & \left.\left\{\exp \left(\alpha^{G} \Delta(n-1) / 4\right), \frac{n+1}{2}\right\}\right) \\
& \leq 1-\left(1-\exp \left(-\alpha^{G} \Delta\left(n-\frac{n+1}{2}\right)\right)\right)^{\exp \left(\alpha^{G} \Delta(n-1) / 4\right)-1} \\
& \leq 1-\left(1-\exp \left(-\alpha^{G} \Delta(n-1) / 2\right)\right)^{\exp \left(\alpha^{G} \Delta(n-1) / 4\right)} \\
& \leq \exp \left(-\alpha^{G} \Delta(n-1) / 4\right)
\end{aligned}
$$

where the last inequality can be derived using Bernoulli's inequality. Using $(n-1) / 4 \geq n / 5$ for $n \geq 5$ finishes the proof.

We now have sufficient knowledge to conclude that the vertices of random shortest path metrics generated from an arbitrary finite simple connected graph $G$ can be partitioned into a small number of clusters with a given maximal diameter. This result is an immediate consequence of Theorem 2.12 and Lemma 3.8 and is given by the following corollary. It is especially useful if the graph $G$ has the property that the parameters $\alpha^{G}$ and $\beta^{G}$ are relatively close to each other.

Corollary 3.9. Let $\mathcal{G}$ be a family of finite simple connected graphs. Then, for any $\Delta>0$ and any $G \in \mathcal{G}$ there exists a partition of the vertices of a random shortest path metric generated from $G$ into clusters, each of diameter at most $4 \Delta$, such that the expected 
number of clusters needed is bounded from above by

$$
2+\frac{2 n}{\exp \left(\alpha^{G} \Delta n / 5\right)}=O\left(1+\frac{n}{\exp \left(\alpha^{G} \Delta n / 5\right)}\right) .
$$

\subsection{Analysis of Heuristics}

In this section we bound the expected approximation ratios of the greedy heuristic for minimum-distance perfect matching, the nearest neighbor and insertion heuristics for the traveling salesman problem, and a trivial heuristic for the $k$ median problem. We also bound the expected running time of the 2-opt heuristic for the traveling salesman problem. We consider these problems on random shortest path metrics generated from an arbitrary fixed undirected connected simple graph $G$ (on $n$ vertices). The results in this section depend on the parameters $\alpha^{G}$ and $\beta^{G}$ as defined in (3.1). In the next section, we will use the results from this section to derive expected approximation ratios for random shortest path metrics generated from Erdős-Rényi random graphs.

The proofs for the greedy matching and nearest neighbor and insertion heuristics for TSP are very alike. The ideas behind them are built upon ideas by Bringmann et al. [15]: we divide the steps of these heuristics into bins, depending on the value which they add to the total distance of our (partial) matching or TSP tour. Using the clustering result (Corollary 3.9) we bound the total contribution of these bins by $O\left(1 / \alpha^{G}\right)$. Combining the following lemma with our observation regarding sums of lightest edge weights in Chapter 2 (Lemma 2.8) we show that the optimal matching or TSP tour has a value of $\Omega\left(1 / \beta^{G}\right)$ with sufficiently high probability.

Lemma 3.10. Let $S_{m}$ denote the sum of the $m$ lightest edge weights in $G$. Then we have $\mathrm{TSP} \geq \mathrm{MM} \geq S_{n / 2}$, where TSP and MM are the total distance of $a$ shortest TSP tour and a minimum-distance perfect matching, respectively.

Proof. The first inequality follows trivially. For the second inequality, consider a minimum-distance perfect matching. Take the union of the shortest path between each matched pair of vertices. This union must contain at least $n / 2$ different edges of $G$. These edges have a total weight of at least $S_{n / 2}$ and at most MM. So, MM $\geq S_{n / 2}$.

The analyses of the running time of 2-opt and the trivial heuristic for $k$-median are generalizations of the corresponding analyses by Bringmann et al. [15] for random shortest path metrics generated from complete graphs to the case of random shortest path metrics generated from an arbitrary graph $G$. 


\subsubsection{Greedy Heuristic for Minimum-Distance Perfect Match- ing}

Recall from Section 1.2.1 that the greedy heuristic for the minimum-distance perfect matching problem starts with an empty matching and iteratively adds a pair of currently unmatched vertices (an 'edge') to the matching such that the distance between them is minimal. Let GR denote the total distance of the matching computed by the greedy heuristic, and let MM denote the total distance of an optimal matching. We show that the greedy heuristic achieves an approximation ratio of $O\left(\beta^{G} / \alpha^{G}\right)$ for random shortest path metrics generated from an arbitrary graph $G$.

Theorem 3.11. For random shortest path metrics generated from an arbitrary graph $G$ we have $\mathbb{E}[\mathrm{GR}]=O\left(1 / \alpha^{G}\right)$.

Proof. Let $\Delta_{i}:=i / \alpha^{G} n$. We put 'edges' that are being added to the greedy matching into bins according to their distance: bin $i$ receives all 'edges' $\{u, v\}$ satisfying $d(u, v) \in\left(4 \Delta_{i-1}, 4 \Delta_{i}\right]$. Let $X_{i}$ denote the number of 'edges' that end up in bin $i$ and set $Y_{i}:=\sum_{k=i}^{\infty} X_{k}$, i.e., $Y_{i}$ denotes the number of 'edges' in the greedy matching with distance at least $4 \Delta_{i-1}$. Observe that $Y_{1}=n / 2$. For $i>1$, by Corollary 3.9. we can partition the vertices in an expected number of at most $O(1+n / \exp ((i-1) / 5))$ clusters (where the constant hidden by the $O$-notation does not depend on $i$ ), each of diameter at most $4 \Delta_{i-1}$. Just before the greedy heuristic adds for the first time an 'edge' of distance more than $4 \Delta_{i-1}$ to the matching, it must be the case that each of these clusters contains at most one unmatched vertex (otherwise the greedy heuristic could have chosen a shorter 'edge' between two vertices in the same cluster). Therefore, we can conclude that $\mathbb{E}\left[Y_{i}\right] \leq O(1+n / \exp ((i-1) / 5))$ for $i>1$. On the other hand, for values of $i$ such that $4 \Delta_{i-1} \geq 2 \ln (n) / \alpha^{G} n$, it follows from Lemma 3.7 that $\mathbb{E}\left[Y_{i}\right] \leq(n / 2) \cdot \mathbb{P}\left(\Delta_{\max }>4 \Delta_{i-1}\right) \leq n^{3-(i-1) / \ln (n)}$.

Now we sum over all bins, bound the length of each 'edge' in bin $i$ by $4 \Delta_{i}$, and subsequently use Fubini's theorem and the derived bounds on $\mathbb{E}\left[Y_{i}\right]$. This yields

$$
\begin{aligned}
\mathbb{E}[\mathrm{GR}] & \leq \sum_{i=1}^{\infty} 4 \Delta_{i} \cdot \mathbb{E}\left[X_{i}\right]=\sum_{i=1}^{\infty} \frac{4}{\alpha^{G} n} \cdot \mathbb{E}\left[Y_{i}\right] \\
& \leq \frac{4}{\alpha^{G} n} \cdot \mathbb{E}\left[Y_{1}\right]+\sum_{i=2}^{2 \ln (n)} \frac{4}{\alpha^{G} n} \cdot \mathbb{E}\left[Y_{i}\right]+\sum_{i=2 \ln (n)}^{\infty} \frac{4}{\alpha^{G} n} \cdot \mathbb{E}\left[Y_{i}\right] \\
& \leq \frac{2}{\alpha^{G}}+\sum_{i=2}^{2 \ln (n)} O\left(\frac{1}{\alpha^{G} n}+\frac{1}{\alpha^{G} e^{(i-1) / 5}}\right)+\sum_{i=2 \ln (n)}^{\infty} \frac{4}{\alpha^{G}} \cdot n^{2-(i-1) / \ln (n)} \\
& =O\left(1 / \alpha^{G}\right)+O\left(1 / \alpha^{G}\right)+O\left(1 / \alpha^{G}\right)=O\left(1 / \alpha^{G}\right),
\end{aligned}
$$

which completes the proof. 
Theorem 3.12. For random shortest path metrics generated from an arbitrary graph $G$ we have $\mathbb{E}\left[\frac{\mathrm{GR}}{\mathrm{MM}}\right]=O\left(\beta^{G} / \alpha^{G}\right)$.

Proof. Let $\hat{c}>0$ be a sufficiently small constant. Then the approximation ratio of the greedy heuristic on random shortest path metrics generated from an arbitrary graph $G$ can be bounded by

$$
\mathbb{E}\left[\frac{\mathrm{GR}}{\mathrm{MM}}\right] \leq \mathbb{E}\left[\frac{\beta^{G} \cdot \mathrm{GR}}{\hat{c}}\right]+\mathbb{P}\left(\mathrm{MM}<\frac{\hat{c}}{\beta^{G}}\right) \cdot O\left(n^{\log _{2}(3 / 2)}\right),
$$

since the worst-case approximation ratio of the greedy heuristic on metric instances is $O\left(n^{\log _{2}(3 / 2)}\right)[54]$. By Theorem 3.11 the first term is $O\left(\beta^{G} / \alpha^{G}\right)$. Combining Lemmas 2.8 and 3.10 , the second term can be bounded from above by $\exp \left(n\left(1+\frac{1}{2} \ln (\hat{c} \cdot O(1))\right)\right) \cdot O\left(n^{\log _{2}(3 / 2)}\right)=o(1)$ since $|E| \leq \beta^{G} n(n-1) / 2$ by definition of $\beta^{G}$ and since $\hat{c}$ is sufficiently small.

\subsubsection{Nearest Neighbor Heuristic for TSP}

The nearest neighbor heuristic is a greedy approach for the traveling salesman problem. Recall from Section 1.2.2 that this heuristic starts with an arbitrary vertex as its current vertex and iteratively builds a TSP tour by traveling from its current vertex to the closest unvisited vertex and adding the corresponding 'edge' to the tour (and at the end closing the tour by going back to its first vertex after all vertices have been visited). Let NN denote the total distance of the TSP tour computed by the nearest neighbor heuristic, and let TSP denote the total distance of an optimal TSP tour. We show that the nearest neighbor heuristic achieves an approximation ratio of $O\left(\beta^{G} / \alpha^{G}\right)$ for random shortest path metrics generated from an arbitrary graph $G$.

Theorem 3.13. For random shortest path metrics generated from an arbitrary graph $G$ we have $\mathbb{E}[\mathrm{NN}]=O\left(1 / \alpha^{G}\right)$.

Proof. Let $\Delta_{i}:=i / \alpha^{G} n$. We put 'edges' that are being added to the nearest neighbor TSP tour into bins according to their distance: bin $i$ receives all 'edges' $\{u, v\}$ satisfying $d(u, v) \in\left(4 \Delta_{i-1}, 4 \Delta_{i}\right]$. Let $X_{i}$ and $Y_{i}$ be defined as in the proof of Theorem 3.11. Observe that $Y_{1}=n$. For $i>1$, by Corollary 3.9, we can partition the vertices in an expected number of at most $O(1+n / \exp ((i-1) / 5))$ clusters (where the constant hidden by the $O$-notation does not depend on $i$ ), each of diameter at most $4 \Delta_{i-1}$. Every time the nearest neighbor heuristic adds an 'edge' of distance more than $4 \Delta_{i-1}$, this must be an 'edge' from a vertex in some cluster $C_{k}$ to a vertex in another cluster $C_{\ell}$, and the tour must have already visited all other vertices in $C_{k}$ (otherwise the nearest neighbor heuristic could have chosen a shorter 'edge' to an unvisited vertex in $C_{k}$ ). Therefore, we can conclude that $\mathbb{E}\left[Y_{i}\right] \leq O(1+n / \exp ((i-1) / 5))$ for $i>1$. On the other hand, for values of $i$ such that $4 \Delta_{i-1} \geq 2 \ln (n) / \alpha^{G} n$, it follows from Lemma 3.7 that $\mathbb{E}\left[Y_{i}\right] \leq n \cdot \mathbb{P}\left(\Delta_{\max }>4 \Delta_{i-1}\right) \leq n^{3-(i-1) / \ln (n)}$. 
Note that (except for $Y_{1}$ ) we have derived exactly the same bounds as in the proof of Theorem 3.11. Using the same calculations as in that proof, it follows now that $\mathbb{E}[\mathrm{NN}]=O\left(1 / \alpha^{G}\right)$.

Theorem 3.14. For random shortest path metrics generated from an arbitrary graph $G$ we have $\mathbb{E}\left[\frac{\mathrm{NN}}{\mathrm{TSP}}\right]=O\left(\beta^{G} / \alpha^{G}\right)$.

The proof of this theorem is similar to that of Theorem 3.12, with the worstcase approximation ratio of the nearest neighbor heuristic on metric instances being $O(\ln (n))$ [56].

\subsubsection{Insertion Heuristics for TSP}

The insertion heuristics are another greedy approach for the traveling salesman problem. Recall from Section 1.2.2 that an insertion heuristic starts with an initial optimal tour on a few vertices that are selected according to some predefined rule $R$, and iteratively chooses (according to the same rule $R$ ) a vertex that is not in the tour yet and inserts this vertex in the current tour such that the total distance of the tour increases the least. Let $I N_{R}$ denote the total distance of the TSP tour computed by the insertion heuristic using rule $R$, and let TSP denote the total distance of an optimal TSP tour. We show that the insertion heuristic for any rule $R$ achieves an approximation ratio of $O\left(\beta^{G} / \alpha^{G}\right)$ for random shortest path metrics generated from an arbitrary graph $G$.

Theorem 3.15. For random shortest path metrics generated from an arbitrary graph $G$ we have $\mathbb{E}\left[\mathrm{IN}_{R}\right]=O\left(1 / \alpha^{G}\right)$.

Proof. Let $\Delta_{i}:=i / \alpha^{G} n$. We put the steps of the insertion heuristic into bins according to the distance they add to the tour: bin $i$ receives all steps with a contribution in the range $\left(8 \Delta_{i-1}, 8 \Delta_{i}\right]$. Let $X_{i}$ and $Y_{i}$ be defined as in the proof of Theorem 3.11. Observe that $Y_{1} \leq n$. For $i>1$, by Corollary 3.9, we can partition the vertices in an expected number of at most $O(1+n / \exp ((i-1) / 5))$ clusters (where the constant hidden by the $O$-notation does not depend on $i$ ), each of diameter at most $4 \Delta_{i-1}$. Every time the contribution of a step of the insertion heuristic is more than $8 \Delta_{i-1}$, this step must add a vertex to the tour that is part of a cluster $C_{k}$ of which no other vertex is in the tour yet (otherwise the contribution of this step would have been less than $\left.8 \Delta_{i-1}\right)$. Therefore, we can conclude that $\mathbb{E}\left[Y_{i}\right] \leq O(1+n / \exp ((i-1) / 5))$ for $i>1$. On the other hand, for values of $i$ such that $8 \Delta_{i-1} \geq 4 \ln (n) / \alpha^{G} n$, it follows from Lemma 3.7 that $\mathbb{E}\left[Y_{i}\right] \leq n \cdot \mathbb{P}\left(\Delta_{\max }>4 \Delta_{i-1}\right) \leq n^{3-(i-1) / \ln (n)}$.

Using the same method as in the proof of Theorem 3.11 (i.e., summing over all bins, bounding the contribution of each step in bin $i$ by $8 \Delta_{i}$ and using Fubini's 
theorem and the derived bounds on $\left.\mathbb{E}\left[Y_{i}\right]\right)$, and adding the expected contribution $\mathbb{E}\left[T_{R}\right]$ of the initial tour, we obtain

$$
\begin{aligned}
\mathbb{E}\left[\mathbb{I N}_{R}\right] & \leq \mathbb{E}\left[T_{R}\right]+\sum_{i=1}^{\infty} 8 \Delta_{i} \cdot \mathbb{E}\left[X_{i}\right]=\mathbb{E}\left[T_{R}\right]+\sum_{i=1}^{\infty} \frac{8}{\alpha^{G} n} \cdot \mathbb{E}\left[Y_{i}\right] \\
& \leq \mathbb{E}\left[T_{R}\right]+\frac{8}{\alpha^{G} n} \cdot \mathbb{E}\left[Y_{1}\right]+\sum_{i=2}^{2 \ln (n)} \frac{8}{\alpha^{G} n} \cdot \mathbb{E}\left[Y_{i}\right]+\sum_{i=2 \ln (n)}^{\infty} \frac{8}{\alpha^{G} n} \cdot \mathbb{E}\left[Y_{i}\right] \\
& \leq \mathbb{E}\left[T_{R}\right]+\frac{8}{\alpha^{G}} \cdot\left(1+\sum_{i=2}^{2 \ln (n)} O\left(\frac{1}{n}+\frac{1}{e^{(i-1) / 5}}\right)+\sum_{i=2 \ln (n)}^{\infty} n^{2-(i-1) / \ln (n)}\right) \\
& =O\left(1 / \alpha^{G}\right),
\end{aligned}
$$

where we used Theorem 3.13 to bound the expected contribution of the initial tour by $\mathbb{E}\left[T_{R}\right] \leq \mathbb{E}[\mathrm{TSP}] \leq \mathbb{E}[\mathrm{NN}] \leq O\left(1 / \alpha^{G}\right)$. Observe that this proof is completely independent of the order in which the vertices are inserted. Thus, it holds for any choice of the rule $R$.

Theorem 3.16. For random shortest path metrics generated from an arbitrary graph $G$ we have $\mathbb{E}\left[\frac{\mathrm{IN}}{\mathrm{TSP}}\right]=O(1)$.

The proof of this theorem is similar to that of Theorem 3.12, with the worstcase approximation ratio of the insertion heuristic (with any rule $R$ ) on metric instances being $O(\ln (n))$ [56].

\subsubsection{Running Time of 2-Opt Heuristic for TSP}

The 2-opt heuristic is an often used local search algorithm for the traveling salesman problem. Recall from Section 1.2.2 that the 2-opt heuristic starts with an initial solution (chosen according to some predefined rule $R$ ) and iteratively improves this solution by applying so-called 2-exchanges (according to the same predefined rule $R$ ) until no improvement can be made by any 2-exchange. In a 2-exchange the heuristic takes two 'edges' $\left\{v_{1}, v_{2}\right\}$ and $\left\{v_{3}, v_{4}\right\}$, where $v_{1}, v_{2}$, $v_{3}, v_{4}$ are visited in this order in the current solution, and replaces them by the two 'edges' $\left\{v_{1}, v_{3}\right\}$ and $\left\{v_{2}, v_{4}\right\}$ to obtain a new solution. The improvement of this 2-exchange is $\delta=d\left(v_{1}, v_{2}\right)+d\left(v_{3}, v_{4}\right)-d\left(v_{1}, v_{3}\right)-d\left(v_{2}, v_{4}\right)$. A solution is called 2-optimal if $\delta \leq 0$ for all possible 2-exchanges.

We provide an upper bound for the expected number of iterations that 2opt needs for random shortest path metrics generated from an arbitrary graph $G$, independently of the rule $R$ used. In the worst-case scenario this number is exponentially large. We show that 2-opt needs at most $O\left(n^{8} \ln ^{3}(n) \beta^{G} / \alpha^{G}\right)$ iterations in expectation for random shortest path metrics generated from an arbitrary graph $G$. 
We start by introducing some additional notation. Let $v_{1}, v_{2}, v_{3}, v_{4} \in V$ such that they represent a 2-exchange with improvement $\delta=d\left(v_{1}, v_{2}\right)+d\left(v_{3}, v_{4}\right)-$ $d\left(v_{1}, v_{3}\right)-d\left(v_{2}, v_{4}\right)$. For $i, j \in\{1,2,3,4\}$ we use $P_{i j}$ to denote the shortest $v_{i}, v_{j}$ path in $G=(V, E)$. Observe that we can write $\delta$ as a linear combination of the weights of the edges on the paths $P_{12}, P_{34}, P_{13}$ and $P_{24}$. We obtain that $\delta=$ $\sum_{e \in E} \gamma_{e} w(e)$ for some $\gamma_{e} \in\{-2,-1,0,1,2\}$. Now, for each $e=\left\{u, u^{\prime}\right\} \in E$ and each $I \subseteq\left\{P_{12}, P_{34}, P_{13}, P_{24}\right\}$ we define $\delta_{i j}^{e, I}$ as follows:

- If $P_{i j} \notin I$, then $\delta_{i j}^{e, I}$ is the length of a shortest path from $v_{i}$ to $v_{j}$ without using $e$.

- If $P_{i j} \in I$, then $\delta_{i j}^{e, I}$ is the minimum of

- the length of a shortest path from $v_{i}$ to $u$ without using $e$ plus the length of a shortest path from $u^{\prime}$ to $v_{j}$ without using $e$ and

- the length of a shortest path from $v_{i}$ to $u^{\prime}$ without using $e$ plus the length of a shortest path from $u$ to $v_{j}$ without using $e$.

Also, define $\delta^{e, I}=\delta_{12}^{e, I}+\delta_{34}^{e, I}-\delta_{13}^{e, I}-\delta_{24}^{e, I}$. Observe that the (random) variables $\delta_{i j}^{e, I}$ and $\delta^{e, I}$ do not depend on $w(e)$. They only depend on $w\left(e^{\prime}\right)$ for all $e^{\prime} \in E \backslash\{e\}$.

Lemma 3.17. Consider a random shortest path metric generated from an arbitrary graph $G=(V, E)$, and let $v_{1}, v_{2}, v_{3}, v_{4} \in V$. Then, for every outcome of the edge weights satisfying $\delta=d\left(v_{1}, v_{2}\right)+d\left(v_{3}, v_{4}\right)-d\left(v_{1}, v_{3}\right)-d\left(v_{2}, v_{4}\right)>0$, there exist an edge $e \in E$ and a set $I \subseteq\left\{P_{12}, P_{34}, P_{13}, P_{24}\right\}$ such that $\delta=\delta^{e, I}+\gamma w(e)$, where $\gamma \in\{-2,-1,1,2\}$ is determined by $e$ and $I$.

Proof. Fix the edge weights arbitrarily (but satisfying $\delta=d\left(v_{1}, v_{2}\right)+d\left(v_{3}, v_{4}\right)-$ $\left.d\left(v_{1}, v_{3}\right)-d\left(v_{2}, v_{4}\right)>0\right)$ and consider the four shortest paths to which these distances correspond $\left(P_{12}, P_{34}, P_{13}, P_{24}\right)$. Recall that we can write $\delta=\sum_{e \in E} \gamma_{e} w(e)$ for some $\gamma_{e} \in\{-2,-1,0,1,2\}$. Since $\delta>0$, there must exist some $e=\left\{u, u^{\prime}\right\} \in$ $E$ such that $\gamma_{e} \neq 0$. Let $e$ be such an edge and let $I \subseteq\left\{P_{12}, P_{34}, P_{13}, P_{24}\right\}$ be the set of all shortest paths under consideration that contain $e$. Using the definition of $\delta^{e, I}$, it follows that $\delta=\delta^{e, I}+\gamma w(e)$ for $\gamma=\gamma_{e} \in\{-2,-1,1,2\}$.

We will refer to the set $I$ as defined in this proof as the set $I$ corresponding to $e$. From the foregoing lemma we can derive that $\delta \in(0, x]$ implies that there exist an edge $e \in E$, a corresponding set $I \subseteq\left\{P_{12}, P_{34}, P_{13}, P_{24}\right\}$ and a corresponding $\gamma \in\{-2,-1,1,2\}$ such that $\delta^{e, I}+\gamma w(e) \in(0, x]$. The following lemma uses this fact to provide an upper bound for the probability that $\delta \in(0, x]$.

Lemma 3.18. Consider a random shortest path metric generated from an arbitrary graph $G=(V, E)$, and let $v_{1}, v_{2}, v_{3}, v_{4} \in V$. Moreover, let $\gamma_{e} \in\{-2,-1,0,1,2\}$ such that $\delta=d\left(v_{1}, v_{2}\right)+d\left(v_{3}, v_{4}\right)-d\left(v_{1}, v_{3}\right)-d\left(v_{2}, v_{4}\right)=\sum_{e \in E} \gamma_{e} w(e)$. Take an arbitrary $e \in E$ with $\gamma_{e} \neq 0$, let $I \subseteq\left\{P_{12}, P_{34}, P_{13}, P_{24}\right\}$ be the corresponding set, 
and set $\gamma=\gamma_{e}$. Then $\mathbb{P}\left(\delta^{e, I}+\gamma w(e) \in(0, x]\right) \leq x$. Moreover, $\mathbb{P}(\delta \in(0, x]) \leq$ $O\left(\beta^{G} n^{2} x\right)$.

Proof. Fix all edge weights except for $w(e)$. Then the value of $\delta^{e, I}$ is deterministically known. Therefore, we have $\delta^{e, I}+\gamma w(e) \in(0, x]$ if and only if $w(e)$ takes a value in an interval of length $x /|\gamma| \leq x$. The first part of the result follows, since $w(e)$ is drawn from $\operatorname{Exp}(1)$ and the density function of this distribution does not exceed 1. Observe that the number of possible choices for $e$ is bounded by $|E| \leq \beta^{G} n(n-1) / 2=O\left(\beta^{G} n^{2}\right)$. The second part of the result follows now using Lemma 3.17 and a union bound.

We now have sufficient knowledge to prove main result of this section.

Theorem 3.19. Let $\mathcal{T}_{R}$ be the number of iterations taken by the 2-opt heuristic (using rule $R$ ) for the traveling salesman problem before reaching a local optimum. For random shortest path metrics generated from an arbitrary graph $G$ we have $\mathbb{E}\left[\mathcal{T}_{R}\right] \leq$ $O\left(n^{8} \ln ^{3}(n) \beta^{G} / \alpha^{G}\right)$.

Proof. Let $\delta_{\min }>0$ be the minimum improvement that can be made by any 2exchange. The total number of different 2-exchanges is $O\left(n^{4}\right)$, so using Lemma 3.18 and a union bound we obtain $\mathbb{P}\left(\delta_{\min } \leq y\right) \leq O\left(\beta^{G} n^{6} y\right)$.

The initial tour has a total distance of at most $n \Delta_{\max }$. Therefore, it follows that $\mathcal{T}_{R} \leq n \Delta_{\max } / \delta_{\min }$. So, $\mathcal{T}_{R}>x$ implies $\Delta_{\max } / \delta_{\min }>x / n$. This event is contained in the union of the events $\Delta_{\max }>4 \ln (x) \ln (n) / \alpha^{G} n$ and $\delta_{\min }<4 \ln (x) \ln (n) / \alpha^{G} x$. By Lemma 3.7 the first event happens with probability at most $n^{2-\ln (x)}$. The second event happens with probability at most $O\left(\beta^{G} n^{6} \ln (n) \ln (x) / \alpha^{G} x\right)$. So, we have

$$
\mathbb{P}\left(\mathcal{T}_{R}>x\right) \leq n^{2-\ln (x)}+O\left(\beta^{G} n^{6} \ln (n) \ln (x) / \alpha^{G} x\right)
$$

The number of iterations taken by the 2-opt heuristic is bounded from above by $n$ !, so we obtain

$$
\begin{aligned}
\mathbb{E}\left[\mathcal{T}_{R}\right]=\sum_{x=0}^{n !} \mathbb{P}\left(\mathcal{T}_{R}>x\right) & \leq 1+\sum_{x=1}^{n !}\left(n^{2-\ln (x)}+O\left(\beta^{G} n^{6} \ln (n) \ln (x) / \alpha^{G} x\right)\right) \\
& \leq 1+O\left(n^{2} \ln (n)\right)+O\left(\beta^{G} n^{6} \ln (n) \ln ^{2}(n !) / \alpha^{G}\right) \\
& =O\left(n^{8} \ln ^{3}(n) \beta^{G} / \alpha^{G}\right),
\end{aligned}
$$

which completes the proof.

From this result we can conclude that the expected running time for the 2-opt heuristic is polynomially bounded for random shortest path metrics generated from any arbitrary connected graph $G$. The following corollary is an immediate consequence of Theorem 3.19 by observing that $\Omega\left(1 / n^{2}\right) \leq \alpha^{G} \leq \beta^{G} \leq 1$ for any connected graph $G$. 
Corollary 3.20. Let $\mathcal{T}_{R}$ be the number of iterations taken by the 2-opt heuristic (using rule R) for the traveling salesman problem before reaching a local optimum. For random shortest path metrics generated from an arbitrary graph $G$ we have $\mathbb{E}\left[\mathcal{T}_{R}\right] \leq$ $O\left(n^{10} \ln ^{3}(n)\right)$.

\subsubsection{Trivial Heuristic for $k$-Median}

In this section we analyse a trivial heuristic for the $k$-median problem. Recall from Section 1.2.3 that this trivial heuristic simply picks $k$ vertices independent of the metric space, e.g., $U=\left\{v_{1}, \ldots, v_{k}\right\}$. Let TR denote the total cost of the solution given by the trivial heuristic, and let ME denote the total cost of an optimal solution to the $k$-median problem. We show that the trivial heuristic on random shortest path metrics generated from an arbitrary graph $G$ achieves an approximation ratio of $O\left(\beta^{G} / \alpha^{G}\right)$ for 'large' $k$ and $\beta^{G} / \alpha^{G}+o\left(\beta^{G} / \alpha^{G}\right)$ for $k$ sufficiently small.

For our analysis, let $U=\left\{v_{1}, \ldots, v_{k}\right\}$ be an arbitrary set of $k$ vertices. Sort the remaining vertices $\left\{v_{k+1}, \ldots, v_{n}\right\}$ in increasing distance from $U$. For $k+1 \leq$ $i \leq n$, let $\rho_{i}:=d\left(v_{i}, U\right)$ be equal to the distance from $U$ to the $(i-k)$-th closest vertex to $U$. Observe that the random variables $\rho_{i}$ are generated by a simple growth process analogous to the one described in the proof of Lemma 2.10 for $\tau_{k}(v)$. Combining this observation with the definition of the parameters $\alpha^{G}$ and $\beta^{G}$ (cf. (3.1)), we can see that

$$
\sum_{j=k}^{i-1} \operatorname{Exp}\left(\beta^{G} j(n-j)\right) \precsim \rho_{i} \precsim \sum_{j=k}^{i-1} \operatorname{Exp}\left(\alpha^{G} j(n-j)\right) .
$$

This in turn implies that $\operatorname{cost}(U):=\sum_{i=k+1}^{n} \rho_{i}$ is stochastically bounded by

$$
\sum_{i=k}^{n-1} \operatorname{Exp}\left(\beta^{G} i\right) \precsim \operatorname{cost}(U) \precsim \sum_{i=k}^{n-1} \operatorname{Exp}\left(\alpha^{G} i\right) .
$$

From this, we can immediately derive bounds for the expected value of the solution returned by the trivial heuristic.

Lemma 3.21. Fix $U \subseteq V$ of size $k$. For random shortest path metrics generated from an arbitrary graph $G$ we have $\mathbb{E}[\mathrm{TR}]=\mathbb{E}[\operatorname{cost}(U)]$ and

$$
\frac{1}{\beta^{G}}\left(\ln \left(\frac{n-1}{k-1}\right)-1\right) \leq \mathbb{E}[\mathrm{TR}] \leq \frac{1}{\alpha^{G}}\left(\ln \left(\frac{n-1}{k-1}\right)+1\right) .
$$

Proof. We have $\left(H_{n-1}-H_{k-1}\right) / \beta^{G}=\sum_{i=k}^{n-1} 1 / \beta^{G} i \leq \mathbb{E}[\mathrm{TR}] \leq \sum_{i=k}^{n-1} 1 / \alpha^{G} i=$ $\left(H_{n-1}-H_{k-1}\right) / \alpha^{G}$. Using $\ln (n) \leq H_{n} \leq \ln (n)+1$ yields the result. 
In order to bound the approximation ratio of the trivial heuristic we need bounds on the probabilities that the total cost of an optimal solution to the $k$ median problem is small and the total cost of the solution given by the trivial heuristic is large. We start by using the distribution of $\sum_{i=k}^{n-1} \operatorname{Exp}\left(\beta^{G} i\right)$ to find an upper bound for the first probability.

Lemma 3.22. The probability density function $f$ of $\sum_{i=k}^{n-1} \operatorname{Exp}\left(\beta^{G} i\right)$ is given by

$$
f(x)=\beta^{G} k \cdot\left(\begin{array}{c}
n-1 \\
k
\end{array}\right) \cdot \exp \left(-\beta^{G} k x\right) \cdot\left(1-\exp \left(-\beta^{G} x\right)\right)^{n-k-1} .
$$

Proof. The distribution corresponds to the $(n-k)$-th smallest element out of $n-1$ independent, exponentially distributed random variables with parameter $\beta^{G}$. The density of this distribution is known [57, Ex. 2.38].

Lemma 3.23. Let $c \geq 1$ be sufficiently large and let $k \leq c^{\prime} n$ for $c^{\prime}=c^{\prime}(c)>0$ sufficiently small. For random shortest path metrics generated from an arbitrary graph $G$ we have

$$
\mathbb{P}\left(\mathrm{ME} \leq \frac{1}{\beta^{G}}\left(\ln \left(\frac{n-1}{k}\right)-\ln \ln \left(\frac{n}{k}\right)-\ln (c)\right)\right) \leq n^{2-c \Theta(1)} .
$$

Proof. We first want a bound for $f(x)$ at $x=\ln ((n-1) / a k) / \beta^{G}$ for sufficiently large $a$ with $1 \leq a \leq(n-1) / k$. For this particular value of $x$, by Lemma 3.22 we have

$$
f(x)=\beta^{G} k \cdot\left(\begin{array}{c}
n-1 \\
k
\end{array}\right) \cdot \frac{(a k)^{k}(n-1-a k)^{n-k-1}}{(n-1)^{n-1}} \leq \beta^{G} k(a e)^{k}\left(1-\frac{a k}{n-1}\right)^{n-k-1},
$$

where we used $\left(\begin{array}{c}n-1 \\ k\end{array}\right) \leq((n-1) e / k)^{k}$ for the inequality. Since $1+x \leq \exp (x)$ and $(n-k-1) /(n-1)=\Theta(1)$ (since $k$ is sufficiently small), we obtain

$$
f(x) \leq \beta^{G} k(a e)^{k} \exp (-a k \Theta(1)) .
$$

Since $a$ is sufficiently large, the first factors (without the $\beta^{G}$ ) are lower order terms that can be hidden by the $\Theta$. This implies that $f(x) \leq \beta^{G} \exp (-a k \Theta(1))$. Substituting $a=(n-1) \exp \left(-\beta^{G} x\right) / k$ into this yields

$$
f(x) \leq \beta^{G} \exp \left(-(n-1) \exp \left(-\beta^{G} x\right) \Theta(1)\right),
$$

which holds for any $x \in\left[0, \ln ((n-1) / b k) / \beta^{G}\right]$ for some sufficiently large $b$ with $1 \leq b \leq(n-1) / k$. Recall that $\operatorname{cost}(U) \succsim \sum_{i=k}^{n-1} \operatorname{Exp}\left(\beta^{G} i\right)$. So, we have

$$
\mathbb{P}\left(\operatorname{cost}(U)<\ln \left(\frac{n-1}{b k}\right) / \beta^{G}\right) \leq \mathbb{P}\left(\sum_{i=k}^{n-1} \operatorname{Exp}\left(\beta^{G} i\right)<\ln \left(\frac{n-1}{b k}\right) / \beta^{G}\right) .
$$


This latter probability is equal to

$$
\begin{aligned}
\int_{0}^{\ln \left(\frac{n-1}{b k}\right) / \beta^{G}} f(x) \mathrm{d} x & =\int_{0}^{\ln \left(\frac{n-1}{b k}\right) / \beta^{G}} f\left(\ln \left(\frac{n-1}{b k}\right) / \beta^{G}-x\right) \mathrm{d} x \\
& \leq \int_{0}^{\ln \left(\frac{n-1}{b k}\right) / \beta^{G}} \beta^{G} \exp \left(-b k \exp \left(\beta^{G} x\right) \Theta(1)\right) \mathrm{d} x \\
& =\int_{0}^{\ln \left(\frac{n-1}{b k}\right)} \exp (-b k \exp (x) \Theta(1)) \mathrm{d} x \\
& \leq \int_{0}^{\infty} \exp (-b k(1+x) \Theta(1)) \mathrm{d} x \leq \exp (-b k \Theta(1)),
\end{aligned}
$$

where for the last step we used the fact that $\int_{0}^{\infty} \exp (-b k x \Theta(1)) \mathrm{d} x=\Theta(1) / b k \leq$ 1 as $b$ is sufficiently large.

In order for ME to be small, there must exist a subset $U \subseteq V$ of size $k$ that has low cost. We bound this probability by taking a union bound, which yields

$$
\begin{aligned}
\mathbb{P}\left(\mathrm{ME}<\frac{1}{\beta^{G}} \ln \left(\frac{n-1}{b k}\right)\right) & =\mathbb{P}\left(\exists U \subseteq V,|U|=k: \operatorname{cost}(U)<\frac{1}{\beta^{G}} \ln \left(\frac{n-1}{b k}\right)\right) \\
& \leq\left(\begin{array}{l}
n \\
k
\end{array}\right) \cdot \mathbb{P}\left(\operatorname{cost}(U)<\frac{1}{\beta^{G}} \ln \left(\frac{n-1}{b k}\right)\right) \\
& \leq\left(\begin{array}{l}
n \\
k
\end{array}\right) \cdot \exp (-b k \Theta(1)) .
\end{aligned}
$$

Set $b=c \ln (n / k)$ for sufficiently large $c$ with $1 \leq c<-\ln \left(c^{\prime}\right) / c^{\prime}$. Then we fulfil the condition that $b$ is sufficiently large with $1 \leq b \leq(n-1) / k$. Combining this with the bound $\left(\begin{array}{l}n \\ k\end{array}\right) \leq(e n / k)^{k}$ yields

$$
\mathbb{P}\left(\mathrm{ME}<\frac{1}{\beta^{G}}\left(\ln \left(\frac{n-1}{k}\right)-\ln \ln \left(\frac{n}{k}\right)-\ln (c)\right)\right) \leq\left(\frac{e n}{k}\right)^{k} \cdot\left(\frac{n}{k}\right)^{-c k \Theta(1)} .
$$

Since $k$ is sufficiently smaller than $n$, we have $e n / k \leq(n / k)^{2}$. Moreover, since also $k \geq 1$, we have $(n / k)^{k} \geq n$, which implies $(n / k)^{2 k-c k \Theta(1)} \leq n^{2-c \Theta(1)}$ since $c$ is sufficiently large. This completes the proof.

Besides the bound in the foregoing lemma on the probability that the total cost of an optimal solution to the $k$-median problem is small, we also need another bound for the probability that the total cost of an optimal solution to the $k$-median problem is much too small, and a bound for the probability that the 
total cost of the solution given by the trivial heuristic is much too large. The following two lemmas provide these bounds.

Lemma 3.24. Let $k \leq(1-\varepsilon) n$ for some constant $\varepsilon>0$. For random shortest path metrics generated from an arbitrary graph $G$ we have

$$
\mathbb{P}\left(\mathrm{ME} \leq c / \beta^{G}\right) \leq\left(\frac{e^{2} c}{2 \varepsilon^{2}}\right)^{\varepsilon n}
$$

for any $c>0$.

Proof. The value of ME is the sum of $n-k$ shortest path lengths in $G$. The union of these paths contains at least $n-k$ different edges from $G$. Let $S_{m}$ be the sum of the $m$ lightest edge weights in $G$. We obtain ME $\geq S_{n-k} \geq S_{\varepsilon n}$. The result follows using Lemma 2.8 and the fact that $|E| \leq \beta^{G} n(n-1) / 2 \leq \beta^{G} n^{2} / 2$.

Lemma 3.25. For random shortest path metrics generated from an arbitrary graph $G$ we have

$$
\mathbb{P}\left(\mathrm{TR}>n^{c}\right) \leq \exp \left(-n^{c / 4}\right)
$$

for any $c \geq 4$.

Proof. We use the (very rough) bounds TR $\leq n \Delta_{\max } \leq n^{2} \max _{e \in E} w(e)$. to bound the value of TR. Furthermore, since $\max _{e \in E} w(e)$ is the maximum of $|E| \leq \beta^{G} n(n-1) / 2$ independent exponentially distributed random variables with parameter 1 , we have

$$
\begin{aligned}
\mathbb{P}\left(\mathrm{TR} \leq n^{\mathcal{c}}\right) & \geq\left(1-\exp \left(-n^{c-2}\right)\right)^{\beta^{G} n(n-1) / 2} \\
& \geq 1-\frac{1}{2} \beta^{G} n(n-1) \cdot \exp \left(-n^{c-2}\right) \\
& \geq 1-\exp \left(-n^{c-3}\right) \geq 1-\exp \left(-n^{c / 4}\right) .
\end{aligned}
$$

The result follows by taking the complement.

Now we have obtained everything needed to provide an upper bound for the expected approximation ratio of the trivial heuristic.

Theorem 3.26. Let $k \leq(1-\varepsilon) n$ for some constant $\varepsilon>0$. For random shortest path metrics generated from an arbitrary graph $G$ we have $\mathbb{E}\left[\frac{\mathrm{TR}}{\mathrm{ME}}\right]=O\left(\beta^{G} / \alpha^{G}\right)$. Moreover, if $k=o(n)$, then we have $\mathbb{E}\left[\frac{\mathrm{TR}}{\mathrm{ME}}\right]=\left(\beta^{G} / \alpha^{G}\right) \cdot(1+o(1))$.

Proof. We have for all $m>0$

$$
\mathbb{E}\left[\frac{\mathrm{TR}}{\mathrm{ME}}\right] \leq \mathbb{E}\left[\frac{\beta^{G} \cdot \mathrm{TR}}{m}\right]+\mathbb{P}\left(\mathrm{ME}<\frac{m}{\beta^{G}}\right) \cdot \mathbb{E}\left[\frac{\mathrm{TR}}{\mathrm{ME}} \mid \mathrm{ME}<\frac{m}{\beta^{G}}\right] .
$$


Case 1 ( $k \leq c^{\prime} n, c^{\prime}$ sufficiently small): Let $n$ de sufficiently large. According to Lemma 3.23 we can pick a constant $c \geq 1$ sufficiently large such that

$$
\mathbb{P}\left(\mathrm{ME} \leq \frac{1}{\beta^{G}}\left(\ln \left(\frac{n-1}{k}\right)-\ln \ln \left(\frac{n}{k}\right)-\ln (c)\right)\right) \leq n^{-9} .
$$

Take $m=\ln ((n-1) / k)-\ln \ln (n / k)-\ln (c)$. By Lemma 3.21, we have

$$
\mathbb{E}\left[\frac{\beta^{G} \cdot \mathrm{TR}}{m}\right] \leq \frac{\beta^{G}}{\alpha^{G}} \cdot \frac{\ln \left(\frac{n-1}{k-1}\right)+1}{m} \leq \frac{\beta^{G}}{\alpha^{G}} \cdot\left(1+O\left(\frac{\ln \ln (n / k)}{\ln (n / k)}\right)\right) .
$$

Note that this final expression can be bounded by $O\left(\beta^{G} / \alpha^{G}\right)$, and that for $k=$ $o(n)$ this can be improved to $\left(\beta^{G} / \alpha^{G}\right) \cdot(1+o(1))$. For the second part we can use the fact that $m$ was chosen such that $\mathbb{P}\left(\mathrm{ME} \leq m / \beta^{G}\right) \leq n^{-9}$ to obtain

$$
\begin{aligned}
\mathbb{P}\left(\mathrm{ME}<\frac{m}{\beta^{G}}\right) \cdot \mathbb{E} & {\left[\frac{\mathrm{TR}}{\mathrm{ME}} \mid \mathrm{ME}<\frac{m}{\beta^{G}}\right] } \\
& =\mathbb{P}\left(\mathrm{ME}<\frac{m}{\beta^{G}}\right) \cdot \int_{0}^{\infty} \mathbb{P}\left(\frac{\mathrm{TR}}{\mathrm{ME}} \geq x \mid \mathrm{ME}<\frac{m}{\beta^{G}}\right) \mathrm{d} x \\
& \leq \mathbb{P}\left(\mathrm{ME}<\frac{m}{\beta^{G}}\right) \cdot\left(n^{8}+\int_{n^{8}}^{\infty} \mathbb{P}\left(\frac{\mathrm{TR}}{\mathrm{ME}} \geq x \mid \mathrm{ME}<\frac{m}{\beta^{G}}\right) \mathrm{d} x\right) \\
& \leq \frac{1}{n}+\int_{n^{8}}^{\infty} \mathbb{P}\left(\frac{\mathrm{TR}}{\mathrm{ME}} \geq x \text { and } \mathrm{ME}<\frac{m}{\beta^{G}}\right) \mathrm{d} x \\
& \leq \frac{1}{n}+\int_{n^{8}}^{\infty} \mathbb{P}\left(\frac{\mathrm{TR}}{\mathrm{ME}} \geq x\right) \mathrm{d} x \\
& \leq \frac{1}{n}+\int_{n^{8}}^{\infty} \mathbb{P}(\mathrm{TR} \geq \sqrt{x}) \mathrm{d} x+\int_{n^{8}}^{\infty} \mathbb{P}\left(\mathrm{ME} \leq \frac{1}{\beta^{G} \sqrt{x}}\right) \mathrm{d} x
\end{aligned}
$$

where the last inequality follows since TR/ME $\geq x$ implies TR $\geq \sqrt{x}$ or ME $\leq$ $1 / \sqrt{x} \leq 1 / \beta^{G} \sqrt{x}$. Note that the requirements for applying Lemmas 3.24 and 
3.25 to the corresponding probabilities are met for any $x \in\left[n^{8}, \infty\right)$. Upon applying those we obtain

$$
\begin{aligned}
\mathbb{P}\left(\mathrm{ME}<\frac{m}{\beta^{\mathrm{G}}}\right) \cdot \mathbb{E}\left[\frac{\mathrm{TR}}{\mathrm{ME}} \mid \mathrm{ME}<\frac{m}{\beta^{\mathrm{G}}}\right] \\
\leq \frac{1}{n}+\int_{n^{8}}^{\infty} \exp \left(-x^{1 / 8}\right) \mathrm{d} x+\int_{n^{8}}^{\infty}\left(\frac{e^{2}}{2 \varepsilon^{2} \sqrt{x}}\right)^{\varepsilon n} \mathrm{~d} x \\
=\frac{1}{n}+O\left(n^{7}\right) \cdot e^{-n}+O\left(n^{8}\right) \cdot\left(\frac{e^{2}}{2 \varepsilon^{2} n^{4}}\right)^{\varepsilon n}=O\left(\frac{1}{n}\right) .
\end{aligned}
$$

Case $2\left(c^{\prime} n<k \leq(1-\varepsilon) n, \varepsilon>0\right)$ : Let $n$ be sufficiently large. We repeat the proof for the previous case, but this time we choose $m$ as a sufficiently small constant ( $m<2 \varepsilon^{2} / e^{2}$ satisfies). Then, by Lemma 3.24, we have $\mathbb{P}\left(\mathrm{ME}<m / \beta^{G}\right) \leq$ $\left(e^{2} m / 2 \varepsilon^{2}\right)^{\varepsilon n} \leq n^{-9}$ (since $n$ is sufficiently large and $m$ is sufficiently small). Furthermore, by Lemma 3.21, we have

$$
\mathbb{E}\left[\frac{\beta^{G} \cdot \mathrm{TR}}{m}\right] \leq \frac{\beta^{G}}{\alpha^{G}} \cdot \frac{\ln \left(\frac{n-1}{k-1}\right)+1}{m}=O\left(\frac{\beta^{G}}{\alpha^{G}}\right),
$$

since $k>c^{\prime} n$. Together with the second part of the first case, this shows the claim.

\subsection{Application to the Erdós-Rényi Random Graph Model}

In this section, we provide the main results of this chapter. We use the results from Section 3.3 and Lemma 3.1 to analyse the performance of several heuristics in random shortest path metrics generated from Erdős-Rényi random graphs.

When a graph $G=(V, E)$ is created by the $G(n, p)$ model, there is a nonzero probability of $G$ being disconnected. In a corresponding random shortest path metric this results in $d(u, v)=\infty$ for any two vertices $u, v \in V$ that are in different components of $G$. Observe that, if this is the case, the identity of indiscernibles, symmetry and the triangle inequality still hold. Thus we still have a metric and we can bound the performance for random shortest path metrics on such a graph from above by the worst-case performance for metric instances.

Using this observation, we can prove the following result for the performance of the greedy heuristic for random shortest path metrics generated from Erdős-Rényi random graphs. 
Theorem 3.27. Let $\varepsilon \in(0,1)$ and $c>9 / \varepsilon^{2}$ be constant. Let $G=(V, E)$ be an instance of the $G(n, p)$ model for $p \geq c \ln (n) / n$ (as $n \rightarrow \infty$ ), and consider the random shortest path metric generated from this graph. Then, we have $\mathbb{E}\left[\frac{\mathrm{GR}}{\mathrm{MM}}\right]=O(1)$.

Proof. Let $\mathcal{E}$ denote the event that the cut parameters of $G$ are bounded by $(1-$ $\varepsilon) p k(n-k) \leq \alpha_{k}^{G} \leq \beta_{k}^{G} \leq(1+\varepsilon) p k(n-k)$ for all $k \in[n]$. Note that $\mathcal{E}$ implies that $(1-\varepsilon) p \leq \alpha^{G} \leq \beta^{G} \leq(1+\varepsilon) p$. It follows that

$$
\begin{aligned}
\mathbb{E}\left[\frac{\mathrm{GR}}{\mathrm{MM}}\right] & \leq \mathbb{E}\left[\frac{\mathrm{GR}}{\mathrm{MM}} \mid \mathcal{E}\right]+\mathbb{E}\left[\frac{\mathrm{GR}}{\mathrm{MM}} \mid \overline{\mathcal{E}}\right] \cdot \mathbb{P}(\overline{\mathcal{E}}) \\
& \leq O\left(\frac{(1+\varepsilon) p}{(1-\varepsilon) p}\right)+O\left(n^{\log _{2}(3 / 2)}\right) \cdot o\left(\frac{1}{n^{2}}\right)=O(1),
\end{aligned}
$$

where we used the results of Theorem 3.12, Lemma 3.1. and the worst-case approximation ratio of the greedy heuristic on metric instances [54].

We continue with the results for the performance of the nearest neighbor and insertion heuristics for random shortest path metrics generated from ErdősRényi random graphs.

Theorem 3.28. Let $\varepsilon \in(0,1)$ and $c>9 / \varepsilon^{2}$ be constant. Let $G=(V, E)$ be an instance of the $G(n, p)$ model for $p \geq c \ln (n) / n$ (as $n \rightarrow \infty$ ), and consider the random shortest path metric generated from this graph. Then, we have $\mathbb{E}\left[\frac{\mathrm{NN}}{\mathrm{TSP}}\right]=O(1)$ and $\mathbb{E}\left[\frac{\mathrm{IN}}{\mathrm{TSP}}\right]=O(1)$.

Proof. Let $\mathcal{E}$ denote the event that the cut parameters of $G$ are bounded by $(1-$ $\varepsilon) p k(n-k) \leq \alpha_{k}^{G} \leq \beta_{k}^{G} \leq(1+\varepsilon) p k(n-k)$ for all $k \in[n]$. Note that $\mathcal{E}$ implies that $(1-\varepsilon) p \leq \alpha^{G} \leq \beta^{G} \leq(1+\varepsilon) p$. It follows that

$$
\begin{aligned}
\mathbb{E}\left[\frac{\mathrm{NN}}{\mathrm{TSP}}\right] & \leq \mathbb{E}\left[\frac{\mathrm{NN}}{\mathrm{TSP}} \mid \mathcal{E}\right]+\mathbb{E}\left[\frac{\mathrm{NN}}{\mathrm{TSP}} \mid \overline{\mathcal{E}}\right] \cdot \mathbb{P}(\overline{\mathcal{E}}) \\
& \leq O\left(\frac{(1+\varepsilon) p}{(1-\varepsilon) p}\right)+O(\ln (n)) \cdot o\left(\frac{1}{n^{2}}\right)=O(1),
\end{aligned}
$$

where we used the results of Theorem 3.14. Lemma 3.1. and the worst-case approximation ratio of the nearest neighbor heuristic on metric instances [56]. For the second part, we use the same argument, which follows this time from the results of Theorem 3.16. Lemma 3.1 and the worst-case approximation ratio of the insertion heuristics on metric instances [56]. Note that this argument is independent of the rule $R$ used.

For the last two results of this chapter, we need the assumption that $G$ is connected. First, we consider the expected running time of the 2-opt heuristic for the traveling salesman problem for random shortest path metrics generated from Erdős-Rényi random graphs. 
Theorem 3.29. Let $\varepsilon \in(0,1)$ and $c>9 / \varepsilon^{2}$ be constant. Let $G=(V, E)$ be an instance of the $G(n, p)$ model for $p \geq c \ln (n) / n$ (as $n \rightarrow \infty)$, and consider the random shortest path metric generated from this graph. If $G$ is connected, then the expected number of iterations of the 2-opt heuristic for the traveling salesman problem is bounded by $O\left(n^{8} \ln ^{3}(n)\right)$.

Proof. Let $\mathcal{T}_{R}$ be the number of iterations taken by the 2-opt heuristic (using rule $R$ ) and let $\mathcal{E}$ denote the event that the cut parameters of $G$ are bounded by $(1-\varepsilon) p k(n-k) \leq \alpha_{k}^{G} \leq \beta_{k}^{G} \leq(1+\varepsilon) p k(n-k)$ for all $k \in[n]$, whereas $\mathcal{E}^{\prime}$ denotes the event that $G$ is connected. Note that $\mathcal{E}$ implies $\mathcal{E}^{\prime}$ and that $\mathcal{E}$ implies that $(1-\varepsilon) p \leq \alpha^{G} \leq \beta^{G} \leq(1+\varepsilon) p$. Moreover, note that the event $\mathcal{E}^{\prime}$ implies that the parameters $\alpha^{G}$ and $\beta^{G}$ are bounded by $\Omega\left(1 / n^{2}\right) \leq \alpha^{G} \leq \beta^{G} \leq 1$. It follows that

$$
\begin{aligned}
\mathbb{E}\left[\mathcal{T}_{R} \mid \mathcal{E}^{\prime}\right] & \leq \mathbb{E}\left[\mathcal{T}_{R} \mid \mathcal{E}^{\prime}, \mathcal{E}\right]+\mathbb{E}\left[\mathcal{T}_{R} \mid \mathcal{E}^{\prime}, \overline{\mathcal{E}}\right] \cdot \mathbb{P}(\overline{\mathcal{E}}) \\
& \leq O\left(n^{8} \ln ^{3}(n) \cdot \frac{(1+\varepsilon) p}{(1-\varepsilon) p}\right)+O\left(n^{8} \ln ^{3}(n) \cdot \frac{1}{1 / n^{2}}\right) \cdot o\left(\frac{1}{n^{2}}\right) \\
& =O\left(n^{8} \ln ^{3}(n)\right),
\end{aligned}
$$

where we used the results of Theorem 3.19 and Lemma 3.1 .

The last result of this chapter concerns the performance of the trivial heuristic for the $k$-median problem for random shortest path metrics generated from Erdős-Rényi random graphs.

Theorem 3.30. Let $\varepsilon \in(0,1)$ and $c>9 / \varepsilon^{2}$ be constant. Let $G=(V, E)$ be an instance of the $G(n, p)$ model for $p \geq c \ln (n) / n$ (as $n \rightarrow \infty$ ), and consider the random shortest path metric generated from this graph. Let $\mathcal{E}^{\prime}$ denote the event that $G$ is connected. Let $k \leq\left(1-\varepsilon^{\prime}\right) n$ for some constant $\varepsilon^{\prime}>0$, then we have $\mathbb{E}\left[\frac{\mathrm{TR}}{\mathrm{ME}} \mid \mathcal{E}^{\prime}\right]=O(1)$. Moreover, if $k=o(n)$, then we have $\mathbb{E}\left[\frac{\mathrm{TR}}{\mathrm{ME}} \mid \mathcal{E}^{\prime}\right]=1+\varepsilon+o(1)$.

Proof. Let $\mathcal{E}$ denote the event that the cut parameters of $G$ are bounded by $(1-$ $\varepsilon) p k(n-k) \leq \alpha_{k}^{G} \leq \beta_{k}^{G} \leq(1+\varepsilon) p k(n-k)$ for all $k \in[n]$. Note that $\mathcal{E}$ implies $\mathcal{E}^{\prime}$ and that $\mathcal{E}$ implies that $(1-\varepsilon) p \leq \alpha^{G} \leq \beta^{G} \leq(1+\varepsilon) p$. Moreover, note that the event $\mathcal{E}^{\prime}$ implies that the parameters $\alpha^{\bar{G}}$ and $\beta^{G}$ are bounded by $\Omega\left(1 / n^{2}\right) \leq$ $\alpha^{G} \leq \beta^{G} \leq 1$. It follows that

$$
\begin{aligned}
\mathbb{E}\left[\frac{\mathrm{TR}}{\mathrm{ME}} \mid \mathcal{E}^{\prime}\right] & \leq \mathbb{E}\left[\frac{\mathrm{TR}}{\mathrm{ME}} \mid \mathcal{E}^{\prime}, \mathcal{E}\right]+\mathbb{E}\left[\frac{\mathrm{TR}}{\mathrm{ME}} \mid \mathcal{E}^{\prime}, \overline{\mathcal{E}}\right] \cdot \mathbb{P}(\overline{\mathcal{E}}) \\
& \leq O\left(\frac{(1+\tilde{\mathcal{E}}) p}{(1-\tilde{\mathcal{E}}) p}\right)+O\left(\frac{1}{1 / n^{2}}\right) \cdot o\left(\frac{1}{n^{2}}\right)=O(1)
\end{aligned}
$$


where we used the results of Theorem 3.26 and Lemma 3.1. Moreover, if $k=$ $o(n)$, then

$$
\begin{aligned}
\mathbb{E}\left[\frac{\mathrm{TR}}{\mathrm{ME}} \mid \mathcal{E}^{\prime}\right] & \leq \mathbb{E}\left[\frac{\mathrm{TR}}{\mathrm{ME}} \mid \mathcal{E}^{\prime}, \mathcal{E}\right]+\mathbb{E}\left[\frac{\mathrm{TR}}{\mathrm{ME}} \mid \mathcal{E}^{\prime}, \overline{\mathcal{E}}\right] \cdot \mathbb{P}(\overline{\mathcal{E}}) \\
& \leq \frac{(1+\tilde{\varepsilon}) p}{(1-\tilde{\varepsilon}) p} \cdot(1+o(1))+O\left(\frac{1}{1 / n^{2}}\right) \cdot(1+o(1)) \cdot o\left(\frac{1}{n^{2}}\right) \\
& =1+\varepsilon+o(1),
\end{aligned}
$$

where $\varepsilon=(1+\tilde{\varepsilon}) /(1-\tilde{\varepsilon})-1$ can be made arbitrarily small by taking $\tilde{\varepsilon}$ sufficiently small, and where we again used the results of Theorem 3.26 and Lemma 3.1 .

\subsection{Coupling Argument}

In this section we present a second approach to obtain (a slightly weaker) version of the results in Section 3.4. In this approach we consider four different variants of random shortest path metrics and show that with high probability these variants are actually similar to scaled versions of each other. In order to distinguish these four variants we use the notation $\mathcal{M}(\mathcal{G}, P)$ where $\mathcal{G}$ denotes the family of graphs used to generate the random shortest path metric, and where $P$ denotes the probability distribution used to generate the (independent) edge weights. We use $\mathcal{G}=K$ to denote the family of complete graphs and $\mathcal{G}=\mathrm{ER}_{p}$ to denote the family of Erdős-Rényi random graphs with probability parameter $p$. Moreover, we use $P=\operatorname{Exp}(1)$ to denote the exponential distribution with parameter 1 and $P=U[0,1]$ to denote the uniform distribution on the interval $[0,1]$.

We show in three steps that many results concerning the distances of 'edges' in the $\mathcal{M}(K, \operatorname{Exp}(1))$ model carry over to the $\mathcal{M}\left(\mathrm{ER}_{p}, \operatorname{Exp}(1)\right)$ model (and vice versa), scaled with a factor $1 / p$.

The first step is a standard coupling argument (e.g. [33]) which shows that with high probability the distances in the $\mathcal{M}(K, \operatorname{Exp}(1))$ model have approximately the same distribution as the distances in the $\mathcal{M}(K, U[0,1])$ model. For the second step we use a similar argument to show that the same result holds if we replace $K$ by ER $\mathrm{ER}_{p}$ with $p=\omega(\ln (n) / n)$. Intuitively, both these couplings follow from the fact that all 'edges' in the considered metric spaces have a length of $o(1)$ w.h.p., and hence only the behaviour of the edge weight distribution in a small interval $[0, o(1)]$ is relevant, because heavier edges are 'ignored' anyway by the shortest paths.

The last step shows that with high probability the distances in the $\mathcal{M}\left(\mathrm{ER}_{p}\right.$, $U[0,1])$ model have the same distribution as the distances in the $\mathcal{M}(K, U[0,1])$ model scaled with a factor $1 / p$. Intuitively, this follows from the fact that an Erdős-Rényi random graph $G=G(n, p)$ can be constructed from a complete 
graph with uniform edge weights on the interval $[0,1]$ by removing all edges with weight larger than $p$.

Due to the nature of the proofs for these coupling arguments we need to be able to easily distinguish the edge weights and 'edge' lengths in the four different random shortest path metric models. The notation that we use (only in this section) is summarized in the table below. Recall that the edge weights correspond to edges from the graph (that is being used to construct the random shortest path metric), whereas the 'edge' lengths correspond to 'edges' from the random metric space. Note that $V_{i j}$ and $T_{i j}$ are only defined for $\{i, j\} \in E$ (where $E$ denotes the edge set of the Erdős-Rényi random graph), whereas the other random variables in this table are defined for all $i, j \in V$ with $i \neq j$.

\begin{tabular}{|c|c|c|c|}
\hline Model & Edge weights & Shortest paths & 'Edge' lengths \\
\hline $\mathcal{M}(K, \operatorname{Exp}(1))$ & $S_{i j} \sim \operatorname{Exp}(1)$ & $P_{i j}$ & $W_{i j}=\sum_{\{k, \ell\} \in P_{i j}} S_{k \ell}$ \\
\hline $\mathcal{M}(K, U[0,1])$ & $U_{i j} \sim U[0,1]$ & $Q_{i j}$ & $X_{i j}=\sum_{\{k, \ell\} \in Q_{i j}} U_{k \ell}$ \\
\hline $\mathcal{M}\left(\mathrm{ER}_{p}, U[0,1]\right)$ & $V_{i j} \sim U[0,1]$ & $Q_{i j}^{\prime}$ & $Y_{i j}=\sum_{\{k, \ell\} \in Q_{i j}^{\prime}} V_{k \ell}$ \\
\hline $\mathcal{M}\left(\mathrm{ER}_{p}, \operatorname{Exp}(1)\right)$ & $T_{i j} \sim \operatorname{Exp}(1)$ & $P_{i j}^{\prime}$ & $Z_{i j}=\sum_{\{k, \ell\} \in P_{i j}^{\prime}} T_{k \ell}$ \\
\hline
\end{tabular}

Let $F(t)=1-e^{-t}$ denote the cumulative distribution function of the (standard) exponential distribution, and let $F^{-1}(t)=-\ln (1-t)$ denote its inverse. Observe that $F^{-1}\left(U_{i j}\right)$ has the same distribution as $S_{i j}$, and that $F^{-1}\left(V_{i j}\right)$ has the same distribution as $T_{i j}$. Therefore, we may assume without loss of generality that $S_{i j}=F^{-1}\left(U_{i j}\right)$ and $T_{i j}=F^{-1}\left(V_{i j}\right)$.

Next, we argue that we also may assume without loss of generality that $V_{i j}=U_{i j} / p$ (for the edges of the Erdős-Rényi random graph). Consider a random instance of the $\mathcal{M}(K, U[0,1])$ model. Observe that $\mathbb{P}\left(U_{i j} \leq p\right)=p$ (independently for each edge $\{i, j\})$. Hence, the set $E:=\left\{\{i, j\}: U_{i j} \leq p\right\}$ is probabilistically equal to the edge set of an Erdős-Rényi random graph with probability $p$. Now, for $\{i, j\} \in E$, it follows that $U_{i j} \sim U[0, p]$ and hence $U_{i j} / p$ has the same distribution as $V_{i j}$. Therefore, we may assume without loss of generality that $V_{i j}=U_{i j} / p$ (for the edges of the Erdős-Rényi random graph).

Using these assumptions and the notation in the table above, we are now ready to formally state the different parts of the coupling arguments that we introduced above. The first two lemmas provide the coupling arguments between the exponential and uniform distributions. We start with the coupling for the models that use complete graphs.

Lemma 3.31. For any constants $\varepsilon \in(0,1)$ and $c>0$ there exists an $N \in \mathbb{N}$ such that for any $n \geq N$, with probability $1-O\left(n^{-c}\right)$ we have

$$
(1-\varepsilon) X_{i j}<W_{i j}<(1+\varepsilon) X_{i j} .
$$


Proof. Let $\varepsilon \in(0,1)$ and $c>0$, and define $a:=(4 c+8) /(1-\varepsilon)$. For the first part of the proof, assume that $W_{i j}<a \ln (n) / n$ for all $i, j \in V$. Then it follows that $0 \leq S_{k \ell}=F^{-1}\left(U_{k \ell}\right)<a \ln (n) / n$ for all edges $\{k, \ell\} \in \bigcup_{i, j \in V} P_{i j}$. Now, since $F^{-1}(t) / t \rightarrow 1$ as $F^{-1}(t) \rightarrow 0$ and since $F^{-1}\left(U_{k \ell}\right) \rightarrow 0$ as $n \rightarrow \infty$, we can derive that $S_{k \ell} / U_{k \ell} \rightarrow 1$ as $n \rightarrow \infty$ for all those edges. So, there exists an $N_{1} \in \mathbb{N}$ such that for any $n \geq N_{1}$ we have $1-\varepsilon<S_{k \ell} / U_{k \ell}<1+\varepsilon$ for all edges $\{k, \ell\} \in \bigcup_{i, j \in V} P_{i j}$. From this, we can derive that for any $i, j \in V$ and any $n \geq N_{1}$ we have

$$
(1-\varepsilon) X_{i j}=\sum_{\{k, \ell\} \in Q_{i j}}(1-\varepsilon) U_{k \ell} \leq \sum_{\{k, \ell\} \in P_{i j}}(1-\varepsilon) U_{k \ell}<\sum_{\{k, \ell\} \in P_{i j}} S_{k \ell}=W_{i j} .
$$

For the second part of the proof, assume that $X_{i j}<a \ln (n) / n$ for all $i, j \in V$. Then it follows that $0 \leq U_{k \ell}<a \ln (n) / n$ for all edges $\{k, \ell\} \in \bigcup_{i, j \in V} Q_{i j}$. Now, since $F^{-1}(t) / t \rightarrow 1$ as $t \rightarrow 0$ and since $U_{k \ell} \rightarrow 0$ as $n \rightarrow \infty$, we can derive that $S_{k \ell} / U_{k \ell} \rightarrow 1$ as $n \rightarrow \infty$ for all those edges. So, there exists an $N_{2} \in \mathbb{N}$ such that for any $n \geq N_{2}$ we have $1-\varepsilon<S_{k \ell} / U_{k \ell}<1+\varepsilon$ for all edges $\{k, \ell\} \in \bigcup_{i, j \in V} Q_{i j}$. From this, we can derive that for any $i, j \in V$ and any $n \geq N_{2}$ we have

$$
W_{i j}=\sum_{\{k, \ell\} \in P_{i j}} S_{k \ell} \leq \sum_{\{k, \ell\} \in Q_{i j}} S_{k \ell}<\sum_{\{k, \ell\} \in Q_{i j}}(1+\varepsilon) U_{k \ell}=(1+\varepsilon) X_{i j} .
$$

Combining both parts, it follows that $(1-\varepsilon) X_{i j}<W_{i j}<(1+\varepsilon) X_{i j}$ if the condition that $W_{i j}<a \ln (n) / n$ and $X_{i j}<a \ln (n) / n$ for all $i, j \in V$ is satisfied. It remains to show that this condition is satisfied with probability $1-O\left(n^{-c}\right)$. Observe that $W_{i j}<(1-\varepsilon) a \ln (n) / n$ for all $i, j \in V$ is sufficient to meet this condition. So, it suffices to show that $\mathbb{P}\left(\exists i, j: W_{i j} \geq(1-\varepsilon) a \ln (n) / n\right) \leq O\left(n^{-c}\right)$. Using the result of Lemma 3.7 and the fact that $\alpha^{G}=1$ for the complete graph, we can see that this probability is bounded by

$$
\mathbb{P}\left(\exists i, j: W_{i j} \geq \frac{(1-\varepsilon) a \ln (n)}{n}\right) \leq n^{2-(1-\varepsilon) a / 4}=n^{-c} .
$$

The result follows.

Next, we show that a similar coupling argument holds for the models that use Erdős-Rényi random graphs. Its proof is a generalization of the proof of the previous lemma.

Lemma 3.32. Let $p=\omega(\ln (n) / n)$. For any constant $\varepsilon \in(0,1)$ there exists an $N \in \mathbb{N}$ such that for any $n \geq N$, with probability $1-o\left(n^{-2}\right)$ we have

$$
(1-\varepsilon) Y_{i j}<Z_{i j}<(1+\varepsilon) Y_{i j}
$$

Proof. Let $\varepsilon \in(0,1)$ and define $a:=17 /(1-\varepsilon)^{2}$. For the first part of the proof, assume that $Z_{i j}<a \ln (n) / n p$ for all $i, j \in V$. Then it follows that $0 \leq T_{k \ell}=$ 
$F^{-1}\left(V_{k \ell}\right)<a \ln (n) / n p$ for all edges $\{k, \ell\} \in \bigcup_{i, j \in V} P_{i j}^{\prime}$. Now, since $F^{-1}(t) / t \rightarrow 1$ as $F^{-1}(t) \rightarrow 0$ and since $F^{-1}\left(V_{k \ell}\right) \rightarrow 0$ as $n \rightarrow \infty$, we can derive that $T_{k \ell} / V_{k \ell} \rightarrow$ 1 as $n \rightarrow \infty$ for all those edges. So, there exists an $N_{1} \in \mathbb{N}$ such that for any $n \geq N_{1}$ we have $1-\varepsilon<T_{k \ell} / V_{k \ell}<1+\varepsilon$ for all edges $\{k, \ell\} \in \bigcup_{i, j \in V} P_{i j}^{\prime}$. From this, we can derive that for any $i, j \in V$ and any $n \geq N_{1}$ we have

$$
(1-\varepsilon) Y_{i j}=\sum_{\{k, \ell\} \in Q_{i j}^{\prime}}(1-\varepsilon) V_{k \ell} \leq \sum_{\{k, \ell\} \in P_{i j}^{\prime}}(1-\varepsilon) V_{k \ell}<\sum_{\{k, \ell\} \in P_{i j}^{\prime}} T_{k \ell}=Z_{i j} .
$$

For the second part of the proof, assume that $Y_{i j}<a \ln (n) / n p$ for all $i, j \in V$. Then it follows that $0 \leq V_{k \ell}<a \ln (n) / n p$ for all edges $\{k, \ell\} \in \bigcup_{i, j \in V} Q_{i j}^{\prime}$. Now, since $F^{-1}(t) / t \rightarrow 1$ as $t \rightarrow 0$ and since $V_{k \ell} \rightarrow 0$ as $n \rightarrow \infty$, we can derive that $T_{k \ell} / V_{k \ell} \rightarrow 1$ as $n \rightarrow \infty$ for all those edges. So, there exists an $N_{2} \in \mathbb{N}$ such that for any $n \geq N_{2}$ we have $1-\varepsilon<T_{k \ell} / V_{k \ell}<1+\varepsilon$ for all edges $\{k, \ell\} \in \bigcup_{i, j \in V} Q_{i j}^{\prime}$. From this, we can derive that for any $i, j \in V$ and any $n \geq N_{2}$ we have

$$
Z_{i j}=\sum_{\{k, \ell\} \in P_{i j}^{\prime}} T_{k \ell} \leq \sum_{\{k, \ell\} \in Q_{i j}^{\prime}} T_{k \ell}<\sum_{\{k, \ell\} \in Q_{i j}^{\prime}}(1+\varepsilon) V_{k \ell}=(1+\varepsilon) Y_{i j} .
$$

Combining both parts, it follows that $(1-\varepsilon) Y_{i j}<Z_{i j}<(1+\varepsilon) Y_{i j}$ if the condition that $Z_{i j}<a \ln (n) / n p$ and $Y_{i j}<a \ln (n) / n p$ for all $i, j \in V$ is satisfied. It remains to show that this condition is satisfied with probability $1-o\left(n^{-2}\right)$. Observe that $Z_{i j}<(1-\varepsilon) a \ln (n) / n p$ for all $i, j \in V$ is sufficient to meet this condition. So, it suffices to show that $\mathbb{P}\left(\exists i, j: Z_{i j} \geq(1-\varepsilon) a \ln (n) / n p\right) \leq o\left(n^{-2}\right)$. This probability can be bounded by

$$
\begin{aligned}
& \mathbb{P}\left(\exists i, j: Z_{i j} \geq \frac{(1-\varepsilon) a \ln (n)}{n p}\right) \\
& \leq \mathbb{P}\left(\exists i, j: Z_{i j} \geq \frac{(1-\varepsilon)^{2} a \ln (n)}{\alpha^{G} n}\right) \\
& \quad+\mathbb{P}\left(\exists i, j: Z_{i j} \geq \frac{(1-\varepsilon) a \ln (n)}{n p} \mid(1-\varepsilon) p \geq \alpha^{G}\right) \cdot \mathbb{P}\left((1-\varepsilon) p \geq \alpha^{G}\right) \\
& \leq n^{2-(1-\varepsilon)^{2} a / 4}+1 \cdot o\left(n^{-2}\right)=o\left(n^{-2}\right),
\end{aligned}
$$

where we used Lemmas 3.1 and 3.7 for the second inequality. The result follows.

The third and final coupling argument shows that (with high probability) the $\mathcal{M}\left(\mathrm{ER}_{p}, U[0,1]\right)$ model is simply a scaled version of the $\mathcal{M}(K, U[0,1])$ model when $p$ is not too small. The following lemma provides this result. 
Lemma 3.33. Let $p=\omega(\ln (n) / n)$. For any constant $c>0$ there exists an $N \in \mathbb{N}$ such that for any $n \geq N$, with probability $1-O\left(n^{-c}\right)$ we have

$$
Y_{i j}=\frac{X_{i j}}{p}
$$

Proof. Let $\varepsilon \in(0,1)$ and $c>0$ and define $a=(4 c+8) /(1-\varepsilon)$. Observe that (by definition) $Q_{i j}^{\prime} \subseteq E$ for all $i, j \in V$. Assume that $X_{i j}<a \ln (n) / n<p$ for all $i, j \in V$. Then we also have $Q_{i j} \subseteq E$ for all $i, j \in V$. In particular, this assumption implies that $Q_{i j}=Q_{i j}^{\prime}$ and hence

$$
Y_{i j}=\sum_{\{k, \ell\} \in Q_{i j}^{\prime}} V_{k \ell}=\sum_{\{k, \ell\} \in Q_{i j}} V_{k \ell}=\sum_{\{k, \ell\} \in Q_{i j}} \frac{U_{k \ell}}{p}=\frac{X_{i j}}{p} .
$$

It remains to show that there exists an $N \in \mathbb{N}$ such that the assumption that $X_{i j} \leq a \ln (n) / n<p$ for all $i, j \in V$ holds with probability $1-O\left(n^{-c}\right)$. Since $p=\omega(\ln (n) / n)$, the second inequality of the assumption holds for sufficiently large $n$. For the first inequality, observe that we can use the results of Lemmas 3.31 and 3.7 (with the fact that $\alpha^{G}=1$ for the complete graph) to see that

$$
\begin{gathered}
\mathbb{P}\left(\exists i, j: X_{i j} \geq \frac{a \ln (n)}{n}\right) \\
\quad \leq \mathbb{P}\left(\exists i, j: W_{i j} \geq \frac{(1-\varepsilon) a \ln (n)}{n}\right)+\mathbb{P}\left(W_{i j} \leq(1-\varepsilon) X_{i j}\right) \\
\leq n^{2-(1-\varepsilon) a / 4}+n^{-c}=2 n^{-c} .
\end{gathered}
$$

The result follows.

Combining the three foregoing lemmas, we can see that $(1-\varepsilon) W_{i j}<p \cdot Z_{i j}<$ $(1+\varepsilon) W_{i j}$ with probability $1-o\left(n^{2}\right)$ for any $\varepsilon>0, p=\omega(\ln (n) / n)$ and $n$ sufficiently large. Loosely speaking, this implies that the $\mathcal{M}\left(\operatorname{ER}_{p}, \operatorname{Exp}(1)\right)$ model is with high probability simply a scaled version of the $\mathcal{M}(K, \operatorname{Exp}(1))$ model. Using this observation, it is possible to derive (a slightly weaker version of) the results in Section 3.4 on the $\mathcal{M}\left(\mathrm{ER}_{p}, \operatorname{Exp}(1)\right)$ model immediately from their corresponding results by Bringmann et al. [15] on the $\mathcal{M}(K, \operatorname{Exp}(1))$ model. 



\section{Chapter 4}

\section{Analysis of Heuristics on RSPM from Sparse Graphs}

Given the fact that most real life networks are sparse, at least intuitively it makes sense to consider random shortest path metrics generated from sparse graphs if we are looking for models that produce random metric spaces which are more realistic. In this chapter we present our results concerning this variant of random shortest path metrics.

Originally, the research for this chapter focused on random shortest path metrics generated from square grid graphs, but later we generalized it to a much wider class of sparse graphs. Some of the results in this chapter even hold for random shortest path metrics generated from any sparse graph. Most results however, are only valid for random shortest path metrics generated from sparse graphs with 'fast growing cut sizes'. We start this chapter by introducing this notion properly.

As in the previous chapter, we then provide some structural properties of the model that we are considering, random shortest path metrics generated from sparse graphs. In the end, these properties enable us to apply the general clustering result from Chapter 2 again.

Next, we use these structural insights to perform a probabilistic analysis for some greedy-like heuristics for the minimum-distance perfect matching problem and the traveling salesman problem. We end this chapter with a probabilistic analysis of the performance of the 2-opt heuristic for the traveling salesman problem. This chapter is based on [41]. 


\subsection{Sparse Graphs}

Throughout this chapter, we consider random shortest path metrics generated from sparse connected undirected simple graphs on $n$ vertices. We have $|E|=$ $\Theta(|V|)=\Theta(n)$ for any sparse graph $G=(V, E)$. The probabilistic analysis of the 2-opt heuristic for the traveling salesman problem in Section 4.4 works for any such graph. However, for the probabilistic analyses of the greedy-like heuristics in Section 4.3 we need to restrict ourselves to classes of sparse graphs that have 'fast growing cut sizes'. The following definition formalizes the notion of fast growing cut sizes.

Definition 4.1. Let $\mathcal{G}$ be a family of sparse connected undirected simple graphs. We say that $\mathcal{G}$ has fast growing cut sizes if there exist constants $c>0$ and $\varepsilon, c^{\prime} \in(0,1)$ such that for any $G=(V, E) \in \mathcal{G}$ and any $U \subseteq V$ with $|U| \leq c^{\prime} n$ we have

$$
\left|\delta_{G}(U)\right| \geq c|U|^{\varepsilon} .
$$

In the remainder of this chapter, whenever we say that a family of sparse graphs has fast growing cut sizes, we implicitly assume that it satisfies Definition 4.1 for some constants $c, \varepsilon, c^{\prime}$.

Intuitively, this definition implies that a family of sparse graphs with fast growing cut sizes cannot have too many 'bottlenecks'. Loosely speaking, a bottleneck is given by two relatively large sets of vertices with only relatively few edges between them.

Note that $c|U|^{\varepsilon}$ is a subadditive function. Hence, when checking whether a family of sparse graphs has fast growing cut sizes, we can restrict ourselves to connected subsets $U \subseteq V$ with $|U| \leq c^{\prime} n$ : if $\left|\delta_{G}(U)\right| \geq c|U|^{\varepsilon}$ for all such connected subsets $U \subseteq V$, then it follows for any unconnected subset $\tilde{U}=$ $U_{1} \cup \ldots \cup U_{k}$ (where the $U_{i}$ are the maximal connected subsets of $\tilde{U}$ ) that

$$
\left|\delta_{G}(\tilde{U})\right|=\sum_{i=1}^{k}\left|\delta_{G}\left(U_{i}\right)\right| \geq \sum_{i=1}^{k} c\left|U_{i}\right|^{\varepsilon} \geq c\left(\sum_{i=1}^{k}\left|U_{i}\right|\right)^{\varepsilon}=c|\tilde{U}|^{\varepsilon} .
$$

Observe that that for graph $G$ in a family of sparse graphs with fast growing cut sizes we can easily bound (one of) the cut parameters of $G$ as defined in Definition 2.9 for such a graph we have $\alpha_{k}^{G} \geq c k^{\varepsilon}$ for $k \leq c^{\prime} n$.

Even though Definition 4.1 might seem rather restrictive at first glance, many graph classes have fast growing cut sizes. In particular, the following example shows that the family of $d$-dimensional grid graphs has fast growing cut sizes. A $d$-dimensional grid graph has vertex set $V=[N]^{d}$, and two vertices $\left(u_{1}, \ldots, u_{d}\right),\left(v_{1}, \ldots, v_{d}\right) \in V$ are connected if and only if $\sum_{i=1}^{d}\left|u_{i}-v_{i}\right|=1$. For such graphs we have $|V|=n=N^{d}$ and $|E|=d N^{d-1}(N-1)=\Theta(n)$.

Example 4.2. For any integer $d>1$, the family of d-dimensional grid graphs has fast growing cut sizes. To see this, let $G=(V, E)$ be a d-dimensional grid graph with 
$n=N^{d}$ vertices. It is known (cf. [13. Thm. 3]) that for any $U \subset V$ with $|U| \leq n / 2$ we have

$$
\left|\delta_{G}(U)\right| \geq \min _{r \in[d]}\left\{r n^{\frac{1}{r}-\frac{1}{d}}|U|^{1-\frac{1}{r}}\right\} .
$$

Exploiting the inequality $|U| \leq n / 2$ (or, equivalently, $n \geq 2|U|$ ) we now obtain that

$$
\left|\delta_{G}(U)\right| \geq \min _{r \in[d]}\left\{r(2|U|)^{\frac{1}{r}-\frac{1}{d}}|U|^{1-\frac{1}{r}}\right\}=\min _{r \in[d]}\left\{r 2^{\frac{1}{r}-\frac{1}{d}}\right\} \cdot|U|^{1-\frac{1}{d}}=2^{1-\frac{1}{d}} \cdot|U|^{1-\frac{1}{d}} .
$$

Hence, for any $d>1$, the family of d-dimensional grid graphs satisfies Definition 4.1 for $c=2^{1-1 / d}, \varepsilon=1-1 / d$ and $c^{\prime}=1 / 2$.

Other examples of families of sparse graphs that have fast growing cut sizes include lattice graphs and random geometric graphs (with high probability). Empirically, also many (real-life) network graphs have fast growing cut sizes.

\subsection{Structural Properties}

In this section we provide some structural properties regarding random shortest path metrics generated from sparse graphs with fast growing cut sizes. These properties are used later on in our probabilistic analyses of the greedy-like heuristics for the minimum-distance perfect matching problem and the traveling salesman problem in such random spaces.

We start by applying the general results concerning a random growth process in Section 2.3.2 to the more specific case of random shortest path metrics generated from sparse graphs with fast growing cut sizes. Then we use this result to derive a specific bound on the distribution of the 'ball-sizes' in such random metric spaces, which we need to be able to apply the clustering result from Section 2.3.3. After that, we also use the random growth process to derive a tail bound for the diameter of the random metric space.

\subsubsection{A Random Growth Process}

As mentioned in Chapter 2, in order to understand the structure of random shortest path metrics it is important to get a feeling for the distribution of the distances in the random metric, in particular the distribution of $\tau_{k}(v)$. The following corollary provides a stochastic bound for the distribution of $\tau_{k}(v)$, which is the immediate result of applying the general random growth process in Section 2.3.2 to sparse graphs with fast growing cut sizes.

Corollary 4.3. Let $\mathcal{G}$ be a family of sparse graphs with fast growing cut sizes. Then, for any $G=(V, E) \in \mathcal{G}$, any $v \in V$ and any $k \leq c^{\prime} n$ we have

$$
\tau_{k}(v) \precsim \sum_{i=1}^{k-1} \operatorname{Exp}\left(c i^{\varepsilon}\right) .
$$


Proof. Observe that for any $G \in \mathcal{G}$ and any $k \leq c^{\prime} n$ we can bound the cut parameter $\alpha_{k}^{G}$ by $\alpha_{k}^{G} \geq c k^{\varepsilon}$ using the definition of fast growing cut sizes. Using the result of Lemma 2.10 we obtain that

$$
\tau_{k}(v) \precsim \sum_{i=1}^{k-1} \operatorname{Exp}\left(\alpha_{i}^{G}\right) \precsim \sum_{i=1}^{k-1} \operatorname{Exp}\left(c i^{\varepsilon}\right) .
$$

Now we use this stochastic upper bound on $\tau_{k}(v)$ to derive some bounds on the cumulative distribution functions of $\tau_{k}(v)$ and $\left|B_{\Delta}(v)\right|$. The final bound on $\left|B_{\Delta}(v)\right|$ enables us to use the powerful clustering result from Section 2.3.3. We start by applying a tail bound for the probability distribution of the generalized Erlang distribution.

Lemma 4.4. Let $\mathcal{G}$ be a family of sparse graphs with fast growing cut sizes. Then, for any $G=(V, E) \in \mathcal{G}$, any $\Delta>0$, any $v \in V$ and any $k \in[n]$ with $k \leq$ $\min \left\{c^{\prime} n,(c(1-\varepsilon) \Delta)^{1 /(1-\varepsilon)}\right\}$ we have

$$
\mathbb{P}\left(\tau_{k}(v) \leq \Delta\right) \geq 1-\frac{H_{k-1}^{(\varepsilon)}}{c \Delta} \cdot \exp \left(-H_{k-1}^{(\varepsilon)}\left(\frac{c \Delta}{H_{k-1}^{(\varepsilon)}}-1-\ln \left(\frac{c \Delta}{H_{k-1}^{(\varepsilon)}}\right)\right)\right) .
$$

Proof. From Corollary 4.3 we can see that

$$
\mathbb{P}\left(\tau_{k}(v) \leq \Delta\right) \geq \mathbb{P}\left(\sum_{i=1}^{k-1} \operatorname{Exp}\left(c i^{\varepsilon}\right) \leq \Delta\right)=1-\mathbb{P}\left(\sum_{i=1}^{k-1} \operatorname{Exp}\left(c i^{\varepsilon}\right) \geq \Delta\right) .
$$

Next, we want to apply the result of Lemma 2.1(i). For this purpose, set

$$
\mu:=\mathbb{E}\left[\sum_{i=1}^{k-1} \operatorname{Exp}\left(c i^{\varepsilon}\right)\right]=\sum_{i=1}^{k-1} \frac{1}{c i^{\varepsilon}}=\frac{H_{k-1}^{(\varepsilon)}}{c} \quad \text { and } \quad \lambda:=\frac{\Delta}{\mu}=\frac{c \Delta}{H_{k-1}^{(\varepsilon)}},
$$

and recall that

$$
\frac{k^{1-\varepsilon}-1}{1-\varepsilon} \leq H_{k-1}^{(\varepsilon)} \leq \frac{k^{1-\varepsilon}}{1-\varepsilon} .
$$

Moreover, since $k \leq(c(1-\varepsilon) \Delta)^{1 /(1-\varepsilon)}$, we can derive that $\lambda=c \Delta / H_{k-1}^{(\varepsilon)} \geq$ $c \Delta(1-\varepsilon) / k^{1-\varepsilon} \geq 1$. Lemma 2.1 (i) now yields

$$
1-\mathbb{P}\left(\sum_{i=1}^{k-1} \operatorname{Exp}\left(c i^{\varepsilon}\right) \geq \Delta\right) \geq 1-\lambda^{-1} \exp (-c \mu(\lambda-1-\ln (\lambda))) .
$$

Finally, we substitute the values of $\mu$ and $\lambda$ to obtain the desired result. 
Next, by observing that $\left|B_{\Delta}(v)\right| \geq k$ if and only if $\tau_{k}(v) \leq \Delta$, we can immediately derive the following corollary.

Corollary 4.5. Let $\mathcal{G}$ be a family of sparse graphs with fast growing cut sizes. Then, for any $G=(V, E) \in \mathcal{G}$, any $\Delta>0$, any $v \in V$ and any $k \in[n]$ with $k \leq$ $\min \left\{c^{\prime} n,(c(1-\varepsilon) \Delta)^{1 /(1-\varepsilon)}\right\}$ we have

$$
\mathbb{P}\left(\left|B_{\Delta}(v)\right|<k\right) \leq \frac{H_{k-1}^{(\varepsilon)}}{c \Delta} \cdot \exp \left(-H_{k-1}^{(\varepsilon)}\left(\frac{c \Delta}{H_{k-1}^{(\varepsilon)}}-1-\ln \left(\frac{c \Delta}{H_{k-1}^{(\varepsilon)}}\right)\right)\right) .
$$

Finally, we use the bound in this corollary to derive a bound of the form that is required for the usage of the powerful clustering result in Theorem 2.12 .

Lemma 4.6. Let $\mathcal{G}$ be a family of sparse graphs with fast growing cut sizes. Then, there exists a constant $c_{1}$ such that for any $\Delta>0$, any $G=(V, E) \in \mathcal{G}$ with $n$ sufficiently large, and any $v \in V$ we have

$$
\mathbb{P}\left(\left|B_{\Delta}(v)\right|<\min \left\{c^{\prime}(c(1-\varepsilon) \Delta)^{1 /(1-\varepsilon)}, c^{\prime} n\right\}\right) \leq \frac{c_{1}}{\Delta^{1 /(1-\varepsilon)}}
$$

Proof. For ease of notation, define $\xi:=c^{\prime}(c(1-\varepsilon))^{1 /(1-\varepsilon)}$ and assume w.l.o.g. that $c_{1} \geq 1 / \xi$. Now observe that for $\Delta \leq 1 / \xi^{1-\varepsilon}$, the statement is trivial since in that case we have $c_{1} / \Delta^{1 /(1-\varepsilon)} \geq c_{1} \xi \geq 1$. So, we are left with the case where $\Delta>1 / \xi^{1-\varepsilon}$.

Let $s_{\Delta}:=\min \left\{\xi \Delta^{1 /(1-\varepsilon)}, c^{\prime} n\right\}$ and observe that $s_{\Delta}>1$ (since $\Delta>1 / \xi^{1-\varepsilon}$ and since $n$ is sufficiently large). Using Corollary 4.5 with $k=s_{\Delta}$ we obtain

$$
\begin{aligned}
\mathbb{P}\left(\left|B_{\Delta}(v)\right|<s_{\Delta}\right) & =\mathbb{P}\left(\left|B_{\Delta}(v)\right|<\left\lceil s_{\Delta}\right\rceil\right) \\
& \leq \frac{H_{s_{\Delta}-1}^{(\varepsilon)}}{c \Delta} \cdot \exp \left(-H_{s_{\Delta}-1}^{(\varepsilon)}\left(\frac{c \Delta}{H_{s_{\Delta}-1}^{(\varepsilon)}}-1-\ln \left(\frac{c \Delta}{H_{s_{\Delta}-1}^{(\varepsilon)}}\right)\right)\right) .
\end{aligned}
$$

So, it remains to show that there exists a constant $c_{1}$ such that for any $\Delta>1 / \xi^{1-\varepsilon}$ we have

$$
\frac{H_{s_{\Delta}-1}^{(\varepsilon)} \Delta^{1 /(1-\varepsilon)}}{c \Delta} \cdot \exp \left(-H_{s_{\Delta^{-1}}}^{(\varepsilon)}\left(\frac{c \Delta}{H_{s_{\Delta}-1}^{(\varepsilon)}}-1-\ln \left(\frac{c \Delta}{H_{s_{\Delta^{-1}}}^{(\varepsilon)}}\right)\right)\right) \leq c_{1} .
$$

To do so, we consider two cases: $\xi \Delta^{1 /(1-\varepsilon)} \leq c^{\prime} n$ and $\xi \Delta^{1 /(1-\varepsilon)} \geq c^{\prime} n$. 
For the first case, suppose that $\xi \Delta^{1 /(1-\varepsilon)} \leq c^{\prime} n$. Then it follows that $s_{\Delta}=$ $\xi \Delta^{1 /(1-\varepsilon)}$, and we need to show that the function

$$
\begin{aligned}
f(\Delta):= & \frac{H_{\xi \Delta^{1 /(1-\varepsilon)}-1}^{(\varepsilon)} \Delta^{1 /(1-\varepsilon)}}{c \Delta} \\
& \quad \times \exp \left(-H_{\xi \Delta^{1 /(1-\varepsilon)}-1}^{(\varepsilon)}\left(\frac{c \Delta}{H_{\xi \Delta^{1 /(1-\varepsilon)}-1}^{(\varepsilon)}}-1-\ln \left(\frac{c \Delta}{H_{\xi \Delta^{1 /(1-\varepsilon)}-1}^{(\varepsilon)}}\right)\right)\right)
\end{aligned}
$$

is bounded from above by a constant. Now, observe that $\lambda-1-\ln (\lambda)$ is an increasing function of $\lambda$ for $\lambda \geq 1$. Combining this with the observation that

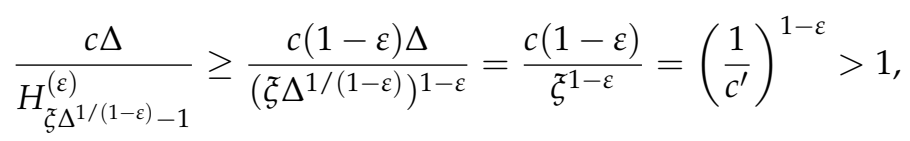

and setting $\gamma:=\left(1 / c^{\prime}\right)^{1-\varepsilon}$ for ease of notation, it follows that

$$
\begin{aligned}
f(\Delta) & \leq \Delta^{1 /(1-\varepsilon)} \cdot \exp \left(-H_{\xi \Delta^{1 /(1-\varepsilon)}-1}^{(\varepsilon)} \cdot(\gamma-1-\ln (\gamma))\right) \\
& \leq \Delta^{1 /(1-\varepsilon)} \cdot \exp \left(-\frac{\xi^{1-\varepsilon} \Delta}{1-\varepsilon} \cdot(\gamma-1-\ln (\gamma))\right),
\end{aligned}
$$

where the second inequality follows by using a standard bound for the generalized harmonic number (cf. (1.1)). It is well-established that the function on the right-hand side has a finite global maximum (since $\gamma>1$ implies $\gamma-1-$ $\ln (\gamma)>0$ ). Therefore, we can conclude that in this case there exists a constant $c_{1}$ such that $f(\Delta) \leq c_{1}$ for all $\Delta>1 / \xi^{1-\varepsilon}$.

For the second case, suppose that $\xi \Delta^{1 /(1-\varepsilon)} \geq c^{\prime} n$. Then it follows that $s_{\Delta}=$ $c^{\prime} n$, and we need to show that the function

$$
g(\Delta, n):=\frac{H_{c^{\prime} n-1}^{(\varepsilon)} \Delta^{1 /(1-\varepsilon)}}{c \Delta} \cdot \exp \left(-H_{c^{\prime} n-1}^{(\varepsilon)}\left(\frac{c \Delta}{H_{c^{\prime} n-1}^{(\varepsilon)}}-1-\ln \left(\frac{c \Delta}{H_{c^{\prime} n-1}^{(\varepsilon)}}\right)\right)\right)
$$

is bounded from above by a constant as long as $\xi \Delta^{1 /(1-\varepsilon)} \geq c^{\prime} n$ and $n$ is sufficiently large. Observe that we can rewrite the inequality $\xi \Delta^{1 /(1-\varepsilon)} \geq c^{\prime} n$ as $c(1-\varepsilon) \Delta \geq n^{1-\varepsilon}$. The first step of the proof is to show that $g(\Delta, n) \leq$ $g\left(n^{1-\varepsilon} /(c(1-\varepsilon)), n\right)$ for all $\Delta \geq n^{1-\varepsilon} /(c(1-\varepsilon))$. To do so, we compute the partial derivative of $g(\Delta, n)$ with respect to $\Delta$, and show that it is non-positive 
for all $\Delta \geq n^{1-\varepsilon} /(c(1-\varepsilon))$. The partial derivative equals

$$
\begin{aligned}
& \frac{\partial g(\Delta, n)}{\partial \Delta}=\frac{H_{c^{\prime} n-1}^{(\varepsilon)} \Delta^{1 /(1-\varepsilon)}}{c(1-\varepsilon) \Delta^{2}} \cdot\left(\varepsilon-c(1-\varepsilon) \Delta+(1-\varepsilon) H_{c^{\prime} n-1}^{(\varepsilon)}\right) \\
& \times \exp \left(-H_{c^{\prime} n-1}^{(\varepsilon)}\left(\frac{c \Delta}{H_{c^{\prime} n-1}^{(\varepsilon)}}-1-\ln \left(\frac{c \Delta}{H_{c^{\prime} n-1}^{(\varepsilon)}}\right)\right)\right) .
\end{aligned}
$$

Now observe that for sufficiently large $n$ we have

$$
c(1-\varepsilon) \Delta \geq n^{1-\varepsilon} \geq \varepsilon+\left(c^{\prime} n\right)^{1-\varepsilon} \geq \varepsilon+(1-\varepsilon) H_{c^{\prime} n-1}^{(\varepsilon)}
$$

where we subsequently used the bound on $\Delta$ for this case, the fact that $n$ is sufficiently large, and a standard bound for the generalized harmonic number for the three inequalities. Together with the facts that $e^{x}>0$ for all $x \in \mathbb{R}$ and $H_{c^{\prime} n-1}^{(\varepsilon)} \Delta^{1 /(1-\varepsilon)} /\left(c(1-\varepsilon) \Delta^{2}\right) \geq 0$, this shows that the partial derivative of $g(\Delta, n)$ with respect to $\Delta$ is indeed non-positive for all $\Delta \geq n^{1-\varepsilon} /(c(1-\varepsilon))$.

Next, notice that $g\left(n^{1-\varepsilon} /(c(1-\varepsilon)), n\right)=f\left(n^{1-\varepsilon} /(c(1-\varepsilon))\right)$. In the first case we have already shown that there exists a constant $c_{1}$ such that $f(\Delta) \leq c_{1}$ for all $\Delta>1 / \xi^{1-\varepsilon}$. So, it follows immediately that $g(\Delta, n) \leq g\left(n^{1-\varepsilon} /(c(1-\varepsilon)), n\right)=$ $f\left(n^{1-\varepsilon} /(c(1-\varepsilon))\right) \leq c_{1}$ as long as $\xi \Delta^{1 /(1-\varepsilon)} \geq c^{\prime} n$ and $n$ is sufficiently large.

Combining both cases yields the desired result.

\subsubsection{Clustering and a Tail Bound for $\Delta_{\max }$}

We now have sufficient knowledge in order to show that the vertices of random shortest path metrics generated from sparse graphs with fast growing cut sizes can be partitioned into a suitably small number of clusters with a given maximum diameter. This result is an immediate consequence of Theorem 2.12 and Lemma 4.6 and is given by the following corollary.

Corollary 4.7. Let $\mathcal{G}$ be a family of sparse graphs with fast growing cut sizes. Then, there exists a constant $c_{1}$ such that for any $\Delta>0$ and any $G \in \mathcal{G}$ there exists a partition of the vertices of a random shortest path metric generated from $G$ into clusters, each of diameter at most $4 \Delta$, such that the expected number of clusters needed is bounded from above by

$$
\frac{1}{c^{\prime}}+\frac{\left(c_{1} \xi+1\right) n}{\xi \Delta^{1 /(1-\varepsilon)}}=O\left(1+\frac{n}{\Delta^{1 /(1-\varepsilon)}}\right)
$$

where $\xi=c^{\prime}(c(1-\varepsilon))^{1 /(1-\varepsilon)}$.

This clustering result is useful as long as $\Delta$ is not too large. However, for large values of $\Delta$, in particular $\Delta \geq \Delta_{\max } / 4$, a 'partition' always requires only one cluster. Recall that $\Delta_{\max }=\max _{u, v} d(u, v)$ is the diameter of the random 
metric space. The probabilistic analyses in the next section need a tail bound on the diameter $\Delta_{\max }$.

For random shortest path metrics generated from complete graphs we know that $\Delta_{\max } \leq O(\log (n) / n)$ with high probability [33]. For random shortest path metrics generated from sparse graphs the diameter is significantly larger. Intuitively this follows from the fact that in a sparse graphs there are significantly fewer different paths between most pairs of vertices compared to the number of different paths in a complete graph. Hence, it becomes significantly less likely to have a really short path between every pair of vertices.

For random shortest path metrics generated from arbitrary graphs, the best possible general bound is $\Delta_{\max } \leq O(n)$ with high probability. Note that for random shortest path metrics generated from a path graph on $n$ vertices, we can easily derive that $\mathbb{E}\left[\Delta_{\max }\right]=\Theta(n)$ (this follows from Corollary 2.7). Hence, the bound in the following lemma is tight.

Lemma 4.8. Let $G=(V, E)$ be an arbitrary connected graph on $n$ vertices and consider a random shortest path metrics generated from $G$. For any $x \geq 6 n$ we have $\mathbb{P}\left(\Delta_{\max } \geq x\right) \leq n e^{-x / 2}$.

Proof. Fix an arbitrary $v \in V$ and let $x \geq 6 n$. We first show that $\mathbb{P}\left(\tau_{n}(v) \geq x\right) \leq$ $e^{-x / 2}$. Since $G$ is connected, we know that $\alpha_{i}^{G} \geq 1$ for all $i \in[n]$. We can derive from Lemma 2.10 that

$$
\tau_{n}(v) \precsim \sum_{i=1}^{n-1} \operatorname{Exp}\left(\alpha_{i}^{G}\right) \precsim \sum_{i=1}^{n-1} \operatorname{Exp}(1) .
$$

From this, we can see that

$$
\mathbb{P}\left(\tau_{n}(v) \geq x\right) \leq \mathbb{P}\left(\sum_{i=1}^{n-1} \operatorname{Exp}(1) \geq x\right)
$$

In order to bound this probability, we once more use Lemma 2.1(i). For this purpose, set

$$
\mu:=\mathbb{E}\left[\sum_{i=1}^{n-1} \operatorname{Exp}(1)\right]=n-1,
$$

and $\lambda:=x / \mu$, and observe that $\lambda \geq 6$ (since $x \geq 6 n$ ). Lemma2.1 (i) now yields

$$
\mathbb{P}\left(\tau_{n}(v) \geq x\right) \leq \lambda^{-1} e^{-\mu(\lambda-1-\ln (\lambda))} \leq e^{-\mu(\lambda / 2)}=e^{-x / 2},
$$

where we used $\lambda-1-\ln (\lambda) \geq \lambda / 2$ (which holds for all $\lambda \geq 5$.36) for the second inequality. The final result follows from observing that $\Delta_{\max }=\max _{v} \tau_{n}(v)$ and applying the appropriate union bound. 


\subsection{Analysis of Greedy-like Heuristics for Matching and TSP}

In this section, we show that three greedy-like heuristics (greedy for minimumdistance perfect matching, and nearest neighbor and insertion for the traveling salesman problem) achieve a constant expected approximation ratio on random shortest path metrics generated from sparse graphs with fast growing cut sizes. The three proofs are very alike, and similar to the corresponding proofs in Chapter 3 The ideas behind them are built upon ideas by Bringmann et al. [15]: we divide the steps of the greedy-like heuristics into bins, depending on the value which they add to the total distance of our (partial) matching or TSP tour. Using the clustering result (Corollary 4.7) we bound the total contribution of these bins by $O(n)$, and using our observations regarding sums of lightest edge weights from previous chapters (Lemmas 2.8 and 3.10) we show that the optimal matching or TSP tour has a value of $\Omega(n)$ with sufficiently high probability.

\subsubsection{Greedy Heuristic for Minimum-Distance Perfect Match- ing}

Recall from Section 1.2.1 that the greedy heuristic for the minimum-distance perfect matching problem starts with an empty matching and iteratively adds a pair of currently unmatched vertices (an 'edge') to the matching such that the distance between them is minimal. Let GR denote the total distance of the matching computed by the greedy heuristic, and let MM denote the total distance of an optimal matching. We show that the greedy heuristic achieves an approximation ratio of $O(1)$ for random shortest path metrics generated from sparse graphs with fast growing cut sizes.

Theorem 4.9. For random shortest path metrics generated from sparse graphs with fast growing cut sizes we have $\mathbb{E}[\mathrm{GR}]=O(n)$.

Proof. We put 'edges' that are being added to the greedy matching into bins according to their distance: bin $i$ receives all 'edges' $\{u, v\}$ satisfying $d(u, v) \in$ $(4(i-1), 4 i]$. Let $X_{i}$ denote the number of 'edges' that end up in bin $i$ and set $Y_{i}:=\sum_{k=i}^{\infty} X_{k}$, i.e., $Y_{i}$ denotes the number of 'edges' in the greedy matching with distance at least $4(i-1)$. Observe that $Y_{1}=n / 2$. For $i>1$, by Corollary 4.7, we can partition the vertices in an expected number of at most $O\left(1+n /(i-1)^{1 /(1-\varepsilon)}\right)$ clusters (where the constant hidden by the O-notation does not depend on $i$ ), each of diameter at most $4(i-1)$. Just before the greedy heuristic adds for the first time an 'edge' of distance more than $4(i-1)$ to the matching, it must be the case that each of these clusters contains at most one unmatched vertex (otherwise the greedy heuristic could have chosen a shorter 'edge' between two vertices in the same cluster). Therefore, we can conclude that $\mathbb{E}\left[Y_{i}\right] \leq O\left(1+n /(i-1)^{1 /(1-\varepsilon)}\right)$ for $i>1$. On the other hand, for values 
of $i$ such that $4(i-1) \geq 6 n$, it follows from Lemma 4.8 that $\mathbb{E}\left[Y_{i}\right] \leq(n / 2)$. $\mathbb{P}\left(\Delta_{\max } \geq 4(i-1)\right) \leq n^{2} e^{-2(i-1)}$.

Now we sum over all bins, bound the length of each 'edge' in bin $i$ by $4 i$, and subsequently use Fubini's theorem and the derived bounds on $\mathbb{E}\left[Y_{i}\right]$. This yields

$$
\begin{aligned}
\mathbb{E}[\mathrm{GR}] & \leq \sum_{i=1}^{\infty} 4 i \cdot \mathbb{E}\left[X_{i}\right]=\sum_{i=1}^{\infty} 4 \cdot \mathbb{E}\left[Y_{i}\right]=4 \cdot \mathbb{E}\left[Y_{1}\right]+\sum_{i=2}^{2 n-1} 4 \cdot \mathbb{E}\left[Y_{i}\right]+\sum_{i=2 n}^{\infty} 4 \cdot \mathbb{E}\left[Y_{i}\right] \\
& \leq 2 n+\sum_{i=2}^{2 n-1} O\left(1+\frac{n}{(i-1)^{1 /(1-\varepsilon)}}\right)+\sum_{i=2 n}^{\infty} 4 n^{2} e^{-2(i-1)} \\
& =O(n)+O(n)+o(1)=O(n),
\end{aligned}
$$

which finishes the proof.

Theorem 4.10. For random shortest path metrics generated from sparse graphs with fast growing cut sizes we have $\mathbb{E}\left[\frac{\mathrm{GR}}{\mathrm{MM}}\right]=O(1)$.

Proof. Let $\hat{c}>0$ be a sufficiently small constant. Then the approximation ratio of the greedy heuristic on random shortest path metrics generated from sparse graphs with fast growing cut sizes can be bounded by

$$
\mathbb{E}\left[\frac{\mathrm{GR}}{\mathrm{MM}}\right] \leq \mathbb{E}\left[\frac{\mathrm{GR}}{\hat{c} n}\right]+\mathbb{P}(\mathrm{MM}<\hat{c} n) \cdot O\left(n^{\log _{2}(3 / 2)}\right),
$$

since the worst-case approximation ratio of the greedy heuristic on metric instances is $O\left(n^{\log _{2}(3 / 2)}\right)$ [54]. By Theorem 4.9 the first term is $O(1)$. Combining Lemmas 2.8 and 3.10 , the second term can be bounded from above by $\exp (n(1+$ $\left.\left.\frac{1}{2} \ln (\hat{c} \cdot \Theta(1))\right)\right) \cdot O\left(n^{\log _{2}(3 / 2)}\right)=o(1)$ since $\hat{c}$ is sufficiently small.

\subsubsection{Nearest Neighbor Heuristic for TSP}

One of the most intuitive heuristics for the traveling salesman problem is the nearest neighbor heuristic. Recall from Section 1.2.2 that this greedy-like heuristic starts with an arbitrary vertex as its current vertex and iteratively builds a TSP tour by traveling from its current vertex to the closest unvisited vertex and adding the corresponding 'edge' to the tour (and at the end closing the tour by going back to its first vertex after all vertices have been visited). Let NN denote the total distance of the TSP tour computed by the nearest neighbor heuristic, and let TSP denote the total distance of an optimal TSP tour. We show that the nearest neighbor heuristic achieves an approximation ratio of $O(1)$ for random shortest path metrics generated from sparse graphs with fast growing cut sizes.

Theorem 4.11. For random shortest path metrics generated from sparse graphs with fast growing cut sizes we have $\mathbb{E}[\mathrm{NN}]=O(n)$. 
Proof. We put 'edges' that are being added to the nearest neighbor TSP tour into bins according to their distance: bin $i$ receives all 'edges' $\{u, v\}$ satisfying $d(u, v) \in(4(i-1), 4 i]$. Let $X_{i}$ and $Y_{i}$ be defined as in the proof of Theorem 4.9. Observe that $Y_{1}=n$. For $i>1$, by Corollary 4.7, we can partition the vertices in an expected number of at most $O\left(1+n /(i-1)^{1 /(1-\varepsilon)}\right)$ clusters (where the constant hidden by the $O$-notation does not depend on $i$ ), each of diameter at most $4(i-1)$. Every time the nearest neighbor heuristic adds an 'edge' of distance more than $4(i-1)$, this must be an 'edge' from a vertex in some cluster $C_{k}$ to a vertex in another cluster $C_{\ell}$, and the tour must have already visited all other vertices in $C_{k}$ (otherwise the nearest neighbor heuristic could have chosen a shorter 'edge' to an unvisited vertex in $C_{k}$ ). Therefore, we can conclude that $\mathbb{E}\left[Y_{i}\right] \leq O\left(1+n /(i-1)^{1 /(1-\varepsilon)}\right)$ for $i>1$. On the other hand, for values of $i$ such that $4(i-1) \geq 6 n$, it follows from Lemma 4.8 that $\mathbb{E}\left[Y_{i}\right] \leq n \cdot \mathbb{P}\left(\Delta_{\max } \geq 4(i-1)\right) \leq n^{2} e^{-2(i-1)}$.

Note that (except for $Y_{1}$ ) we have derived exactly the same bounds as in the proof of Theorem 4.9 Using the same calculations as in that proof, it follows now that $\mathbb{E}[\mathrm{NN}]=\mathrm{O}(n)$.

Theorem 4.12. For random shortest path metrics generated from sparse graphs with fast growing cut sizes we have $\mathbb{E}\left[\frac{\mathrm{NN}}{\mathrm{TSP}}\right]=\mathrm{O}(1)$.

The proof of this theorem is similar to that of Theorem 4.10 , with the worstcase approximation ratio of the nearest neighbor heuristic on metric instances being $O(\ln (n))[56]$.

\subsubsection{Insertion Heuristics for TSP}

Another group of greedy-like heuristics for the traveling salesman problem are the insertion heuristics. Recall from Section 1.2.2 that an insertion heuristic starts with an initial optimal tour on a few vertices that are selected according to some predefined rule $R$, and iteratively chooses (according to the same rule $R$ ) a vertex that is not in the tour yet and inserts this vertex in the current tour such that the total distance of the tour increases the least. Let $I_{R}$ denote the total distance of the TSP tour computed by the insertion heuristic using rule $R$, and let TSP denote the total distance of an optimal TSP tour. We show that the insertion heuristic for any rule $R$ achieves an approximation ratio of $O(1)$ for random shortest path metrics generated from sparse graphs with fast growing cut sizes.

Theorem 4.13. For random shortest path metrics generated from sparse graphs with fast growing cut sizes we have $\mathbb{E}\left[\mathrm{IN}_{R}\right]=O(n)$.

Proof. We put the steps of the insertion heuristic into bins according to the distance they add to the tour: bin $i$ receives all steps with a contribution in the range $(8(i-1), 8 i]$. Let $X_{i}$ and $Y_{i}$ be defined as in the proof of Theorem 4.9 Observe that $Y_{1} \leq n$. For $i>1$, by Corollary 4.7, we can partition the vertices in an expected number of at most $O\left(1+n /(i-1)^{1 /(1-\varepsilon)}\right)$ clusters (where 
the constant hidden by the O-notation does not depend on $i$ ), each of diameter at most $4(i-1)$. Every time the contribution of a step of the insertion heuristic is more than $8(i-1)$, this step must add a vertex to the tour that is part of a cluster $C_{k}$ of which no other vertex is in the tour yet (otherwise the contribution of this step would have been less than $8(i-1))$. Therefore, we can conclude that $\mathbb{E}\left[Y_{i}\right] \leq O\left(1+n /(i-1)^{1 /(1-\varepsilon)}\right)$ for $i>1$. On the other hand, for values of $i$ such that $8(i-1) \geq 6 n$, it follows from Lemma 4.8 that $\mathbb{E}\left[Y_{i}\right] \leq n \cdot \mathbb{P}\left(\Delta_{\max } \geq 4(i-1)\right) \leq n^{2} e^{-2(i-1)}$.

Using the same method as in the proof of Theorem 4.9 (i.e., summing over all bins, bounding the contribution of each step in bin $i$ by $8 i$ and using Fubini's theorem and the derived bounds on $\left.\mathbb{E}\left[Y_{i}\right]\right)$, and adding the expected contribution $\mathbb{E}\left[T_{R}\right]$ of the initial tour, we obtain

$$
\begin{aligned}
\mathbb{E}\left[\mathrm{IN}_{R}\right] & \leq \mathbb{E}\left[T_{R}\right]+\sum_{i=1}^{\infty} 8 i \cdot \mathbb{E}\left[X_{i}\right]=\mathbb{E}\left[T_{R}\right]+\sum_{i=1}^{\infty} 8 \cdot \mathbb{E}\left[Y_{i}\right] \\
& =\mathbb{E}\left[T_{R}\right]+8 \cdot \mathbb{E}\left[Y_{1}\right]+\sum_{i=2}^{n-1} 8 \cdot \mathbb{E}\left[Y_{i}\right]+\sum_{i=n}^{\infty} 8 \cdot \mathbb{E}\left[Y_{i}\right] \\
& \leq O(n)+8 n+\sum_{i=2}^{n-1} O\left(1+\frac{n}{(i-1)^{1 /(1-\varepsilon)}}\right)+\sum_{i=n}^{\infty} 8 n^{2} e^{-2(i-1)}=O(n),
\end{aligned}
$$

where we used Theorem 4.11 to bound the expected contribution of the initial tour by $\mathbb{E}\left[T_{R}\right] \leq \mathbb{E}[\mathrm{TSP}] \leq \mathbb{E}[\mathrm{NN}]=O(n)$. Observe that this proof is independent of the choice of rule $R$.

Theorem 4.14. For random shortest path metrics generated from sparse graphs with fast growing cut sizes we have $\mathbb{E}\left[\frac{\mathrm{IN} \mathrm{N}_{R}}{\mathrm{TSP}}\right]=\mathrm{O}(1)$.

The proof of this theorem is similar to that of Theorem 4.10, with the worstcase approximation ratio of the insertion heuristic (with any rule $R$ ) on metric instances being $O(\ln (n))$ [56].

\subsection{Analysis of 2-Opt for TSP}

In this section, we consider the arguably most famous local search heuristic for the traveling salesman problem, the 2-opt heuristic, and show that it achieves a constant approximation for random shortest path metrics generated from any sparse graph. Note that in this section we do not require the sparse graphs to have fast growing cut sizes.

Recall from Section 1.2.2 that the 2-opt heuristic starts with an arbitrary initial solution and iteratively improves this solution by applying so-called 2exchanges until no improvement is possible anymore. In a 2-exchange the heuristic takes two 'edges' $\left\{u_{1}, v_{1}\right\}$ and $\left\{u_{2}, v_{2}\right\}$, where $u_{1}, v_{1}, u_{2}, v_{2}$ are visited in this order in the current solution, and replaces them by the two 'edges' $\left\{u_{1}, u_{2}\right\}$ 
and $\left\{v_{1}, v_{2}\right\}$ to obtain a new solution. The improvement of this 2-exchange is $\delta=d\left(u_{1}, v_{1}\right)+d\left(u_{2}, v_{2}\right)-d\left(u_{1}, u_{2}\right)-d\left(v_{1}, v_{2}\right)$. A solution is called 2-optimal if $\delta \leq 0$ for all possible 2-exchanges.

The actual performance of the 2-opt heuristic strongly depends on the choice of the initial solution and the sequence of improvements. Our results only concern the worst possible outcome of the 2-opt heuristic, as others have been doing before (see e.g. [18, 22]), since this decouples the actual heuristic from the initialization and therefore keeps the analysis tractable. Let WLO denote the total distance of the worst 2-optimal TSP tour, and let TSP denote the total distance of an optimal TSP tour. We show that the 2-opt heuristic achieves an approximation ratio of $O(1)$ for random shortest path metrics generated from sparse graphs.

The crucial observation that enables us to show this result is the fact that for any 2-optimal solution for the traveling salesman problem it holds that each edge $e \in E$ can appear at most twice in the disjoint union of all shortest paths that belong to this solution. In other words, the total distance of any 2-optimal solution can be bounded by twice the sum of all edge weights in $G$. The following lemma and theorems formalize this observation and its consequences.

In this lemma and these theorems we consider the TSP tours as being directed and use the following notation. For each $i, j \in V$, let $P_{i j}$ denote the set of all (directed) edges in the shortest $i, j$-path.

Lemma 4.15. Let $G=(V, E)$ be an arbitrary connected graph and consider a random shortest path metric generated from this graph. Also, let $\mathcal{S}$ denote an arbitrary 2-optimal solution for the traveling salesman problem on this random metric. Moreover, let $x_{i j}:=$ 1 if this solution $\mathcal{S}$ travels directly from vertex $i$ to vertex $j$, and $x_{i j}:=0$ otherwise. Then, for any $i, j, k, l \in V$ with $x_{i j}=x_{k l}=1$ we have either $P_{i j} \cap P_{k l}=\varnothing$ or $(i, j)=(k, l)$.

Proof. Let $i, j, k, l \in V$ such that $x_{i j}=x_{k l}=1$, and suppose that $(i, j) \neq(k, l)$. Set $A:=\{i, j, k, l\}$ and observe that $|A|$ equals either 3 or 4 . $(|A|=2$ would imply $(i, j)=(k, l)$.)

We first look at the case where $|A|=4$. Suppose, by way of contradiction, that $P_{i j} \cap P_{k l} \neq \varnothing$. Take $e=(s, t) \in P_{i j} \cap P_{k l}$. Then $d(i, j)=d(i, s)+w(e)+d(t, j)$ and $d(k, l)=d(k, s)+w(e)+d(t, l)$. Moreover, using the triangle inequality, we can see that $d(i, k) \leq d(i, s)+d(s, k)$ and $d(j, l) \leq d(j, t)+d(t, l)$. Let $\delta=$ $\delta(i, j, k, l)$ denote the improvement of the 2-exchange where $\{i, j\}$ and $\{k, l\}$ are replaced by $\{i, k\}$ and $\{j, l\}$. Note that $\delta \leq 0$ since $\mathcal{S}$ is a 2-optimal solution for the traveling salesman problem. It follows that

$$
\begin{aligned}
& 0 \geq \delta=d(i, j)+d(k, l)-d(i, k)-d(j, l) \\
& \geq d(i, s)+w(e)+d(t, j)+d(k, s)+w(e)+d(t, l) \\
& -d(i, s)-d(s, k)-d(j, t)-d(t, l) \\
& =2 w(e)>0,
\end{aligned}
$$


which clearly is a contradiction. Therefore, we must have $P_{i j} \cap P_{k l}=\varnothing$ in this case.

Now, we look at the case where $|A|=3$. Since the $x$ variables describe a solution to the traveling salesman problem, this implies that either $j=k$ or $i=l$. These cases are analogous, so w.l.o.g. we assume that $j=k$. The proof that $P_{i j} \cap P_{k l}=\varnothing$ in this case is similar to the proof for $|A|=4$, with the exception that here we have $\delta=d(i, j)+d(j, l)-d(i, j)-d(j, l)=0$ (instead of $\delta \leq 0)$. The desired result follows.

Theorem 4.16. For random shortest path metrics generated from sparse graphs with fast growing cut sizes we have $\mathbb{E}[\mathrm{WLO}]=O(n)$.

Proof. Let $x_{i j}=1$ if WLO travels directly from vertex $i$ to vertex $j$, and $x_{i j}=0$ otherwise. From Lemma 4.15 we know that each edge $e \in E$ can appear at most twice in the disjoint union of all shortest $i, j$-paths that form a 2-optimal tour (at most once per direction). This yields

$$
\mathrm{WLO}=\sum_{i, j \in V} d(i, j) x_{i j}=\sum_{\substack{i, j \in V \\ x_{i j}=1}} \sum_{e \in P_{i j}} w(e) \leq \sum_{e \in E} 2 w(e)=2 S_{|E|},
$$

where $S_{m}$ denotes the sum of the $m$ lightest edge weights in $G$ (as in Chapter2). Combining this with Corollary 2.7. it follows that

$$
\mathbb{E}[\mathrm{WLO}] \leq \mathbb{E}\left[2 S_{|E|}\right]=O\left(\frac{|E|^{2}}{|E|}\right)=O(n),
$$

where the last equality follows by recalling that $|E|=\Theta(n)$ for sparse graphs.

Theorem 4.17. For random shortest path metrics generated from sparse graphs with fast growing cut sizes we have $\mathbb{E}\left[\frac{\mathrm{WLO}}{\mathrm{TSP}}\right]=O(1)$.

The proof of this theorem is similar to that of Theorem 4.10, with the worstcase approximation ratio of the 2-opt heuristic on metric instances being $O(\sqrt{n})$ [18]. 


\section{Chapter 5}

\section{Analysis of Facility Location on RSPM from Complete Graphs}

In this chapter we return to random shortest path metrics generated from complete graphs. In contrast to the previous chapters where we analysed multiple heuristics for several combinatorial optimization problems, in this chapter we focus on only one simple heuristic for one combinatorial optimization problem: the facility location problem.

The heuristic that we investigate opens the $\kappa$ cheapest facilities (breaking ties arbitrarily), where $\kappa$ only depends on the number of vertices and the facility opening costs. We start by introducing this heuristic and argue intuitively why it is likely to perform 'well'.

After also introducing several technical lemmas and two bounds related to the optimal solution, we continue with the main results concerning the performance of this heuristic. We show that it is asymptotically optimal if the facility openings costs are such that the number of facilities that are being opened by the heuristic is not too large $(\kappa=o(n))$, and also provide a closed-form expression as an upper bound for the expected approximation ratio in case the facility opening costs are such that the number of facilities being opened by the heuristic is large $(\kappa=\Theta(n))$. In the special where all facility opening costs are equal, we show that this closed-form expression is $O(\sqrt[4]{\ln (n)})$, which can be improved to $O(1)$ or even $1+o(1)$ when the facility opening costs are sufficiently small. This chapter is based on [40]. 


\subsection{Additional notation}

In this chapter we consider the (uncapacitated) facility location problem as introduced in Section 1.2.4. Recall that we use $f_{i}>0$ to denote the opening costs of a facility at vertex $v_{i}$. We assume without loss of generality that $0<f_{1} \leq \ldots \leq f_{n}$. Moreover, we assume that these opening costs are predetermined, independent of the random edge weights used to define the random metric space.

In this chapter we use $F_{k}:=\sum_{i=1}^{k} f_{i}$ as a shorthand notation for the sum of the opening costs of the $k$ cheapest facilities. Additionally, we assume that the ratios between the opening costs are polynomially bounded, i.e., we assume $f_{n} / f_{1} \leq n^{q}$ for some constant $q$ as $n \rightarrow \infty$.

As mentioned in Section 1.2.4 the objective of the facility location problem is to find a non-empty subset $U \subseteq V$ such that the total cost $c(U)=$ $f(U)+\sum_{v \in V} \min _{u \in U} d(v, u)$ is minimal among all non-empty subsets $U \subseteq V$, where $f(U)$ denotes the total opening cost of facilities at all vertices in $U$. In this chapter, we use OPT to denote the total cost of an optimal solution to this problem, i.e.,

$$
\mathrm{OPT}=\min \{c(U): \varnothing \neq U \subseteq V\} .
$$

One of the tools we use in our proofs in Section 5.5 involves fixing the number of facilities that has to be opened. We use OPT $k$ to denote the total cost of the best solution to the facility location problem with the additional constraint that exactly $k$ facilities need to be opened, i.e.,

$$
\mathrm{OPT}_{k}=\min \{c(U): \varnothing \neq U \subseteq V,|U|=k\} .
$$

Observe that OPT $=\min _{k \in[n]} \mathrm{OPT}_{k}$ by these definitions.

\subsection{A Simple Heuristic and Some of Its Properties}

In this chapter we are interested in a rather simple heuristic that only takes the facility opening costs $f_{i}$ into account while determining which facilities to open and which not, independent of the metric space. Define $\kappa:=\kappa\left(n ; f_{1}, \ldots, f_{n}\right)=$ $\max \left\{i \in[n]: f_{i}<1 /(i-1)\right\}$. Then our heuristic opens the $\kappa$ cheapest facilities (breaking ties arbitrarily). Note that in the special case where all opening costs are the same, i.e., $f_{1}=\ldots=f_{n}=f$, this corresponds to $\kappa=\min \{\lceil 1 / f\rceil, n\}$.

This rather particular value of $\kappa$ originates from the following intuitive argument. Based on the results of Bringmann et al. [15. Lemma 5.1] we know that the expected cost of the solution that opens the $k$ cheapest facilities is given by $g(k):=F_{k}+H_{n-1}-H_{k-1}$. This convex function decreases as long as $k$ satisfies $f_{k}<1 /(k-1)$. Therefore, at least intuitively, the value of $\kappa$ that we use is likely to provide a relatively 'good' solution.

\footnotetext{
${ }^{*}$ The same result can be derived from (the proof of Lemma 3.21 by recalling that $\alpha^{G}=\beta^{G}=1$ for complete graphs.
} 
We show that this is indeed the case. Our main result is split into two parts, based on the actual value of $\kappa$. We show that our simple heuristic is asymptotically optimal for any polynomially bounded opening costs that satisfy $\kappa=o(n)$ (i.e., there are 'many' relatively expensive facilities). On the other hand, if $\kappa=\Theta(n)$, then the analysis becomes more difficult, and we only provide a closed-form expression that can be used to determine an upper bound for the expected approximation ratio. We show that this expression yields an $O(\sqrt[4]{\ln (n)})$ approximation ratio in the special case where $f_{1}=\ldots=f_{n}=f$, and $O(1)$ or even $1+o(1)$ if $f$ is sufficiently small.

Throughout the remainder of this chapter we use ALG to denote the value of the solution provided by this simple heuristic.

\subsubsection{Probability Distribution of ALG}

In this section we derive the probability distribution of the value of the solution provided by our simple greedy heuristic, ALG, and derive its expectation.

If $\kappa=n$, then ALG denotes the cost of the solution which opens a facility at every vertex $v \in V$. So, we have $\mathrm{ALG}=F_{n}$, and, in particular, $\mathbb{P}\left(\mathrm{ALG}=F_{n}\right)=1$.

If $1 \leq \kappa<n$, then the distribution of ALG is less trivial. In this case, the total opening costs are given by $F_{\kappa}$, whereas the distribution of the connection costs is known and given by $\sum_{i=\kappa}^{n-1} \operatorname{Exp}(i)\left[15\right.$, Sect. 5] This results in ALG $-F_{\mathcal{K}} \sim$ $\sum_{i=\kappa}^{n-1} \operatorname{Exp}(i)$.

Using this probability distribution, we can derive the expected value of ALG. If $\kappa=n$, then it follows trivially that $\mathbb{E}[\mathrm{ALG}]=F_{n}$. If $1 \leq \kappa<n$, then we have

$$
\mathbb{E}[\mathrm{ALG}]=F_{\kappa}+\sum_{i=\kappa}^{n-1} \frac{1}{i}=F_{\kappa}+H_{n-1}-H_{\kappa-1}=F_{\kappa}+\ln (n / \kappa)+\Theta(1) .
$$

We end this section with an upper bound for the value of $F_{\kappa}$. By definition of $\kappa$, it follows that $f_{\kappa}<1 /(\kappa-1)$ and thus $F_{\kappa}<\kappa /(\kappa-1) \leq 2$ whenever $\kappa \geq 2$. If $\kappa=1$, then we obviously have $F_{\mathcal{K}}=f_{1}$. Combining these bounds, we see that $F_{\mathcal{K}} \leq \max \left\{2, f_{1}\right\}$.

\subsection{Technical Lemmas}

In this section we present some technical lemmas that are being used for the proofs of our theorems in Section 5.5. These lemmas do not provide new structural insights, but are nonetheless very helpful for our proofs.

First of all, we will use the Cauchy-Schwarz inequality to bound the expected approximation ratio of our simple greedy heuristic. For general random variables $X, Y$ this inequality states that $|\mathbb{E}[X Y]| \leq \sqrt{\mathbb{E}\left[X^{2}\right] \mathbb{E}\left[Y^{2}\right]}$.

\footnotetext{
${ }^{\dagger}$ The same result can be derived from the analysis of $\operatorname{cost}(U)$ in Section 3.3 .5 by recalling that $\alpha^{G}=\beta^{G}=1$ for complete graphs.
} 
Secondly, we want to have an upper bound for the expectation of the maximum of a number of (dependent) random variables. The following lemma provides such an upper bound.

Lemma 5.1 ([4. Thm. 2.1]). Let $X_{1}, \ldots, X_{n}$ be a sequence of random variables, each with finite mean and variance. Then it follows that

$$
\mathbb{E}\left[\max _{i} X_{i}\right] \leq \max _{i} \mathbb{E}\left[X_{i}\right]+\sqrt{\frac{n-1}{n} \cdot \sum_{i=1}^{n} \operatorname{Var}\left(X_{i}\right)} .
$$

We will also make use of the so-called 'Rényi's representation' [52, 55] in order to be able to link sums and order statistics of exponentially distributed random variables. Recall that if $X_{1}, \ldots, X_{m}$ are $m$ random variables, then $X_{(1)}, \ldots$, $X_{(m)}$ are the order statistics corresponding to $X_{1}, \ldots, X_{m}$ if $X_{(i)}$ is the $i$ th smallest value among $X_{1}, \ldots, X_{m}$ for all $i \in[m]$. Rényi's representation states the following.

Lemma 5.2. Let $X_{i} \sim \operatorname{Exp}(\lambda)$ independently for $i=1, \ldots, m$, and let $X_{(1)}, \ldots, X_{(m)}$ be the order statistics corresponding to $X_{1}, \ldots, X_{m}$. Then, jointly for all $i \in[m]$ we have

$$
X_{(i)}=\frac{1}{\lambda} \sum_{j=1}^{i} \frac{Z_{j}}{m-j+1},
$$

where $Z_{j} \sim \operatorname{Exp}(1)$ independently, and where "=" means equal distribution.

A special case of Rényi's representation is given by the following corollary.

Corollary 5.3. Let $Y_{i} \sim \operatorname{Exp}(1)$ independently for $i=1, \ldots, n-1$, and let $Y_{(1)}, \ldots$, $Y_{(n-1)}$ be the order statistics corresponding to $Y_{1}, \ldots, Y_{n-1}$. Then, jointly for all $i \in$ $[n-1]$ we have

$$
Y_{(n-i)} \sim \sum_{k=i}^{n-1} \operatorname{Exp}(k)
$$

Finally, we will make use of the so-called 'Padé approximants' for the (generalized) exponential integral [49. Sec. 14.7]. For the exponential integral, we will use the first four Padé approximants. These are listed below and hold for 
any $z>0$.

$$
\begin{aligned}
& \frac{z}{z+1} \leq z e^{z} \int_{z}^{\infty} \frac{e^{-t}}{t} \mathrm{~d} t \leq \frac{z+1}{z+2} \\
& \frac{z^{2}+3 z}{z^{2}+4 z+2} \leq z e^{z} \int_{z}^{\infty} \frac{e^{-t}}{t} \mathrm{~d} t \leq \frac{z^{2}+5 z+2}{z^{2}+6 z+6} \\
& z e^{z} \int_{z}^{\infty} \frac{e^{-t}}{t} \mathrm{~d} t \leq \frac{z^{3}+11 z^{2}+26 z+6}{z^{3}+12 z^{2}+36 z+24} \\
& z e^{z} \int_{z}^{\infty} \frac{e^{-t}}{t} \mathrm{~d} t \geq \frac{z^{4}+15 z^{3}+58 z^{2}+50 z}{z^{4}+16 z^{3}+72 z^{2}+96 z+24}
\end{aligned}
$$

We also use two Padé approximants for two different generalized exponential integrals. These are listed below and hold for any $z>0$, and any $k>1$ or $k>3$, respectively.

$$
\begin{aligned}
& \frac{z^{2}+(k+1) z}{z^{2}+2 k z+k(k-1)} \leq z^{k-1} e^{z} \int_{z}^{\infty} \frac{e^{-t}}{t^{k-1}} \mathrm{~d} t \leq \frac{z^{2}+(k+3) z+2}{z^{2}+2(k+1) z+k(k+1)} \\
& z^{k-3} e^{z} \int_{z}^{\infty} \frac{e^{-t}}{t^{k-3}} \mathrm{~d} t \\
& \leq \frac{z^{4}+(3 k+7) z^{3}+3\left(k^{2}+3 k+6\right) z^{2}+(k+3)\left(k^{2}-k+10\right) z+24}{z^{4}+4(k+1) z^{3}+6 k(k+1) z^{2}+4 k\left(k^{2}-1\right) z+k\left(k^{2}-1\right)(k-2)}
\end{aligned}
$$

\subsection{Bounds for the Optimal Solution}

Not much is known about the distribution of the value of the optimal solution, OPT. Therefore, in this section we derive two bounds related to the optimal solution which we can use in Section 5.5 .

Recall that OPT $=\min \{c(U): \varnothing \neq U \subseteq V\}$ where $c(U)=f(U)+$ $\sum_{v \in V} \min _{u \in U} d(v, u)$ denotes the total cost of the solution that opens all facilities in $U$. We start with an upper bound for the cumulative distribution function of OPT that works good for relative small values of OPT (i.e., values close to $F_{1}$ ).

Lemma 5.4. Let $z \in\left[F_{1}, F_{n}\right]$ and define $\zeta:=\max \left\{k: z \geq F_{k}\right\}$. Then, for any given opening costs $f_{i}$, we have

$$
\mathbb{P}(\text { OPT }<z) \leq \sum_{i=1}^{\zeta}\left(\begin{array}{c}
n \\
i
\end{array}\right)\left(\begin{array}{c}
n-1 \\
i-1
\end{array}\right)\left(1-e^{-\left(z-F_{i}\right)}\right)^{n-i}
$$


Proof. Let $L$ denote the number of open facilities in the optimal solution (if there are multiple optimal solutions, pick one arbitrarily). If OPT $<z$, then we know that $L=i$ for some $i \in[\zeta]$. Since these cases are disjoint, we can condition as follows:

$$
\mathbb{P}(\mathrm{OPT}<z)=\sum_{i=1}^{\zeta} \mathbb{P}(\mathrm{OPT}<z \mid L=i) \cdot \mathbb{P}(L=i) \leq \sum_{i=1}^{\zeta} \mathbb{P}(\mathrm{OPT}<z \mid L=i) .
$$

Recall that $f(U)=\sum_{j \in U} f_{j}$ is the total opening cost of all facilities in $U$. Using the union bound, we can derive that

$$
\begin{aligned}
\mathbb{P}(\mathrm{OPT}<z \mid L=i) & =\mathbb{P}(\exists U \subseteq V,|U|=i: c(U)<z) \\
& \leq \mathbb{P}\left(\exists U \subseteq V,|U|=i: c(U)-f(U)<z-F_{i}\right) \\
& \leq\left(\begin{array}{c}
n \\
i
\end{array}\right) \cdot \mathbb{P}\left(\sum_{k=i}^{n-1} \operatorname{Exp}(k)<z-F_{i}\right),
\end{aligned}
$$

since $F_{i} \leq f(U)$ and $c(U)-f(U) \sim \sum_{k=i}^{n-1} \operatorname{Exp}(k)$ for all $U \subseteq V$ with $|U|=i$.

Let $Y_{i} \sim \operatorname{Exp}(1)$ independently for $i=1, \ldots, n-1$ and let $Y_{(i)}$ denote the corresponding order statistics. Then, using Rényi's representation (see Corollary 5.3, we can derive that

$$
\mathbb{P}\left(\sum_{k=i}^{n-1} \operatorname{Exp}(k)<z-F_{i}\right)=\mathbb{P}\left(Y_{(n-i)}<z-F_{i}\right) .
$$

Again using the union bound, it follows that

$$
\begin{aligned}
\mathbb{P}\left(Y_{(n-i)}<z-F_{i}\right) & =\mathbb{P}\left(\exists J \subseteq[n-1],|J|=n-i: \max _{j \in J} Y_{j}<z-F_{i}\right) \\
& \leq\left(\begin{array}{c}
n-1 \\
i-1
\end{array}\right) \cdot \mathbb{P}\left(\max _{j \in[n-i]} Y_{j}<z-F_{i}\right) \\
& =\left(\begin{array}{c}
n-1 \\
i-1
\end{array}\right)\left(1-e^{-\left(z-F_{i}\right)}\right)^{n-i} .
\end{aligned}
$$

By combining the results above, the desired result follows immediately.

One of the approaches that we use in our proofs in Section 5.5 involves looking at the optimal solution of the facility location problem with the additional constraint that we have to open exactly $k$ facilities. We use $\mathrm{OPT}_{k}$ to denote the total cost of this modified problem, i.e., $\mathrm{OPT}_{k}=\min \{c(U): \varnothing \neq U \subseteq V,|U|=$ $k\}$. Note that OPT $=\min _{k \in[n]} \mathrm{OPT}_{k}$. The following lemma provides a stochastic lower bound for $\mathrm{OPT}_{n-k}$.

Lemma 5.5. Let $Z_{k} \sim \sum_{i=1}^{k} \operatorname{Exp}\left(e\left(\begin{array}{l}n \\ 2\end{array}\right) / k\right)$. Then we have $\mathrm{OPT}_{n-k} \succsim F_{n-k}+Z_{k}$. 
Proof. If the number of open facilities in a solution is fixed to be $n-k$, then the total opening costs of the optimal solution is trivially lower bounded by $F_{n-k}$. Moreover, the total connection costs in this case are lower bounded by the total length of the $k$ shortest edges in the metric. This in turn can be lower bounded by the total weight of the $k$ lightest edge weights used to generate the metric, which we denote by $S_{k}$ (as in Chapter 2). Using Lemma 2.6, we can see that $S_{k} \succsim Z_{k}$, since $|E|=\left(\begin{array}{l}n \\ 2\end{array}\right)$ for the complete graph. The desired result follows now immediately.

\subsection{Main Results}

In this section we present our main results from this chapter. We show that our simple heuristic is asymptotically optimal if $\kappa=o(n)$ (Theorem 5.6), and we provide a closed-form expression as an upper bound for the expected approximation ratio if $\kappa=\Theta(n)$ (Theorem 5.13). Finally, we evaluate this expression for the special case where $f_{1}=\ldots=f_{n}=f$ (Corollary 5.16).

\subsubsection{Opening a few facilities}

We start with the first theorem, which shows that our simple heuristic is asymptotically optimal if $\kappa=o(n)$.

Theorem 5.6. Define $\kappa:=\kappa\left(n ; f_{1}, \ldots, f_{n}\right)=\max \left\{i \in[n]: f_{i}<1 /(i-1)\right\}$. Let ALG denote the total cost of the solution that opens, independent of the metric space, the $\kappa$ cheapest facilities (breaking ties arbitrarily), i.e., the facilities with opening costs $f_{1}, \ldots, f_{\kappa}$. If $\kappa=o(n)$, then for random shortest path metrics generated from complete graphs we have $\mathbb{E}\left[\frac{\mathrm{ALG}}{\mathrm{OPT}}\right]=1+o(1)$.

In order to prove this theorem, we consider the following three cases for the opening cost $f_{1}$ of the cheapest facility:

1. $f_{1} \leq 1 / \ln ^{2}(n)$ as $n \rightarrow \infty$;

2. $f_{1}=O(\ln (n))$ and $f_{1}>1 / \ln ^{2}(n)$ as $n \rightarrow \infty$;

3. $f_{1}=\omega(\ln (n))$.

We start with the rather straightforward proof of Case 3 in which the total connection costs in expectation are negligible compared to the opening cost of the cheapest facility.

Proof of Theorem 5.6 (Case 3). For sufficiently large $n$ we have $f_{1}>1$, and thus $\kappa=1$ since $f_{2} \geq f_{1}>1=1 /(2-1)$. Therefore, using our observations in Section 5.2.1. we can derive that $\mathbb{E}[\mathrm{ALG}]=F_{1}+\ln (n)+\Theta(1)$ for sufficiently 
large $n$. Moreover, we know that $\mathrm{OPT} \geq F_{1}$. Using this observation, it follows that

$$
\mathbb{E}\left[\frac{\mathrm{ALG}}{\mathrm{OPT}}\right] \leq \mathbb{E}\left[\frac{\mathrm{ALG}}{F_{1}}\right]=\frac{F_{1}+\ln (n)+\Theta(1)}{F_{1}}=1+\frac{\ln (n)+\Theta(1)}{\omega(\ln (n))}=1+o(1),
$$

which finishes the proof of this case.

We continue with Case 1 of Theorem 5.6. Its proof requires two lemmas. The first one shows that the probability that the value of ALG is 'large' in this case is sufficiently small.

Lemma 5.7. Let $f_{1} \leq 1 / \ln ^{2}(n)$ as $n \rightarrow \infty$ and assume that $\kappa<n$. For sufficiently large $n$ we have

$$
\int_{1 / f_{1}^{2}}^{\infty} \mathbb{P}(\mathrm{ALG} \geq \sqrt{x}) \mathrm{d} x \leq O\left(\frac{1}{n}\right) .
$$

Proof. We start by providing a bound for the cumulative distribution function of ALG. Let $t \in \mathbb{R}$. By our observations in Section 5.2.1 we know the distribution of ALG, and since $F_{\mathcal{K}} \leq \max \left\{2, f_{1}\right\}=2$ we can bound it as follows:

$$
\mathbb{P}(\mathrm{ALG} \geq t)=\mathbb{P}\left(\sum_{i=\kappa}^{n-1} \operatorname{Exp}(i) \geq t-F_{\kappa}\right) \leq \mathbb{P}\left(\sum_{i=\kappa}^{n-1} \operatorname{Exp}(i) \geq t-2\right) .
$$

Now, let $Y_{i} \sim \operatorname{Exp}(1)$ independently for $i=1, \ldots, n-1$, and let $Y_{(i)}$ denote the corresponding order statistics. Using Rényi's representation (see Corollary 5.3, we can now rewrite the last probability as follows:

$$
\begin{aligned}
\mathbb{P}\left(\sum_{i=\kappa}^{n-1} \operatorname{Exp}(i) \geq t-2\right) & =\mathbb{P}\left(Y_{(n-k)} \geq t-2\right) \\
& =\mathbb{P}\left(\exists J \subseteq[n-1],|J|=\kappa: \min _{j \in J} Y_{j} \geq t-2\right) .
\end{aligned}
$$

Applying a union bound to this result, we obtain that

$$
\mathbb{P}(\mathrm{ALG} \geq t) \leq\left(\begin{array}{c}
n-1 \\
\kappa
\end{array}\right) \cdot \mathbb{P}\left(\min _{j \in[\kappa]} Y_{j} \geq t-2\right) \leq\left(\begin{array}{c}
n-1 \\
\kappa
\end{array}\right) \cdot e^{-\kappa(t-2)} .
$$

Note that the last inequality becomes an equality whenever $t-2 \geq 0$. We can use the derived bound on the cumulative distribution function of ALG to bound 
the given integral as follows:

$$
\begin{aligned}
\int_{1 / f_{1}^{2}}^{\infty} \mathbb{P}(\mathrm{ALG} \geq \sqrt{x}) \mathrm{d} x & \leq \int_{1 / f_{1}^{2}}^{\infty}\left(\begin{array}{c}
n-1 \\
\kappa
\end{array}\right) \cdot e^{-\kappa(\sqrt{x}-2)} \mathrm{d} x \\
& =\left(\begin{array}{c}
n-1 \\
\kappa
\end{array}\right) \cdot e^{2 \kappa}\left(\frac{2}{\kappa^{2}}\left(1+\frac{\kappa}{f_{1}}\right) e^{-\kappa / f_{1}}\right) \\
& \leq 2 n^{\kappa}\left(1+\frac{1}{f_{1}}\right) e^{3 \kappa-\kappa / f_{1}},
\end{aligned}
$$

where we used $\left(\begin{array}{c}n-1 \\ \kappa\end{array}\right) \leq(e n)^{\kappa}$ to bound the binomial coefficient. It remains to be shown that $2 n^{\kappa}\left(1+1 / f_{1}\right) e^{3 \kappa-\kappa / f_{1}}=O(1 / n)$. To do so, we start by claiming that the following inequality holds for sufficiently large $n$ :

$$
(\kappa+1) \ln (n)+\ln \left(1+\frac{1}{f_{1}}\right)+3 \kappa \leq \frac{\kappa}{f_{1}} .
$$

To see this, observe that for sufficiently large $n$ we have $(\kappa+1) \ln (n) \leq \kappa / 3 f_{1}$, $\ln \left(1+1 / f_{1}\right) \leq \kappa / 3 f_{1}$ and $3 \kappa \leq \kappa / 3 f_{1}$ (in all three cases since $1 / f_{1} \geq \ln ^{2}(n)$ ). Rearranging the inequality, we get

$$
\kappa \ln (n)+\ln \left(1+\frac{1}{f_{1}}\right)+3 \kappa-\frac{\kappa}{f_{1}} \leq-\ln (n) .
$$

Upon exponentiation of both sides we obtain the desired result, which finishes this proof.

The second lemma that we need for the proof of Case 1 of Theorem 5.6 shows that the bound on the cumulative distribution function of OPT from Lemma 5.4 is sufficiently small for a specific value of $z$.

Lemma 5.8. Let $\beta_{n}=\ln (n / \kappa)(1+1 / n)^{-1}$, define $\zeta_{n}:=\max \left\{k: \beta_{n} \geq F_{k}\right\}$ and assume that $\kappa=o(n)$. For sufficiently large $n$, and for any $i \in\left[\zeta_{n}\right]$, we have

$$
\left(\begin{array}{c}
n \\
i
\end{array}\right)\left(\begin{array}{c}
n-1 \\
i-1
\end{array}\right)\left(1-e^{-\left(\beta_{n}-F_{i}\right)}\right)^{n-i} \leq e^{-n} .
$$

Proof. Let $n$ be sufficiently large. Since $i \ln (e n / i)$ is an increasing function of $i$ for all $i \in(0, n)$, it follows that

$$
2 i \ln \left(\frac{e n}{i}\right)-\left(n-\zeta_{n}\right) e^{F_{i}-\beta_{n}} \leq 2 \zeta_{n} \ln \left(\frac{e n}{\zeta_{n}}\right)-\left(n-\zeta_{n}\right) e^{-\beta_{n}},
$$

where we also used the fact that $e^{F_{i}} \geq 1$ for all $i \in[n]$. Next, define $\alpha_{n}:=n / \kappa$ and recall that (by definition of $\kappa$ ) we have $f_{\kappa+c} \geq f_{\kappa+1} \geq 1 / \kappa$ for all $c \geq 1$ and 
thus $F_{\kappa+c}>c / \kappa$. Using this observation, we can see that $F_{\kappa+\left\lceil\kappa \beta_{n}\right\rceil}>\beta_{n}$, from which follows that $\zeta_{n} \leq \kappa\left(1+\beta_{n}\right)$. Using the fact that $\beta_{n} \leq \ln (n / \kappa)=\ln \left(\alpha_{n}\right)$, we can conclude that $\zeta_{n} / n \leq\left(1+\beta_{n}\right) / \alpha_{n} \leq\left(1+\ln \left(\alpha_{n}\right)\right) / \alpha_{n}$. Applying this final inequality, we obtain

$$
\begin{aligned}
2 i \ln \left(\frac{e n}{i}\right)- & \left(n-\zeta_{n}\right) e^{F_{i}-\beta_{n}} \\
& \leq n \cdot\left(\frac{2 \zeta_{n}}{n} \ln \left(\frac{e n}{\zeta_{n}}\right)-\left(1-\frac{\zeta_{n}}{n}\right) e^{-\beta_{n}}\right) \\
& \leq n \cdot\left(\frac{2+2 \ln \left(\alpha_{n}\right)}{\alpha_{n}} \ln \left(\frac{e \alpha_{n}}{1+\ln \left(\alpha_{n}\right)}\right)-\left(1-\frac{1+\ln \left(\alpha_{n}\right)}{\alpha_{n}}\right) e^{-\beta_{n}}\right),
\end{aligned}
$$

since $0<\zeta_{n} / n \leq\left(1+\ln \left(\alpha_{n}\right)\right) / \alpha_{n} \leq 1$. Using the definition of $\beta_{n}$, we can see that $e^{-\beta_{n}}=\alpha_{n}^{1-1 /(n+1)}$. Applying this yields

$$
\begin{aligned}
2 i \ln \left(\frac{e n}{i}\right)- & \left(n-\zeta_{n}\right) e^{F_{i}-\beta_{n}} \\
& \leq n \cdot\left(\frac{2+2 \ln \left(\alpha_{n}\right)}{\alpha_{n}} \ln \left(\frac{e \alpha_{n}}{1+\ln \left(\alpha_{n}\right)}\right)+\frac{1+\ln \left(\alpha_{n}\right)}{\alpha_{n}^{1 /(n+1)}}-\alpha_{n}^{1-1 /(n+1)}\right) .
\end{aligned}
$$

Since $\kappa=o(n)$, it follows by definition that $\alpha_{n}=n / \kappa=\omega(1)$. Observe that the dominant term between the brackets on the right-hand side of the last inequality is given by $-\alpha_{n}^{1-1 /(n+1)}$ (since the first term converges to 0 as $\alpha_{n} \rightarrow \infty$ and the second term is (at most) logarithmic in $\alpha_{n}$ ). This implies that this factor becomes less than -1 whenever $n$ is sufficiently large. So, we obtain that

$$
2 i \ln \left(\frac{e n}{i}\right)-\left(n-\zeta_{n}\right) e^{F_{i}-\beta_{n}} \leq-n .
$$

Combining this with the well-known inequality $\ln (1-x) \leq-x$ (for $0 \leq x<1$ ), it follows that

$$
2 i \ln \left(\frac{e n}{i}\right)+\left(n-\zeta_{n}\right) \ln \left(1-e^{-\left(\beta_{n}-F_{i}\right)}\right) \leq 2 i \ln \left(\frac{e n}{i}\right)-\left(n-\zeta_{n}\right) e^{F_{i}-\beta_{n}} \leq-n .
$$

From this inequality, we immediately get

$$
\left(\frac{e n}{i}\right)^{2 i} \cdot\left(1-e^{-\left(\beta_{n}-F_{i}\right)}\right)^{n-\zeta_{n}} \leq e^{-n}
$$


On the other hand, since $\left(\begin{array}{c}n-1 \\ k-1\end{array}\right) \leq\left(\begin{array}{l}n \\ k\end{array}\right) \leq(e n / k)^{k}$ and $\left(\begin{array}{l}n_{1} \\ k_{1}\end{array}\right)\left(\begin{array}{l}n_{2} \\ k_{2}\end{array}\right) \leq\left(\begin{array}{l}n_{1}+n_{2} \\ k_{1}+k_{2}\end{array}\right)$, it follows also that

$$
\begin{aligned}
\left(\begin{array}{c}
n \\
i
\end{array}\right)\left(\begin{array}{c}
n-1 \\
i-1
\end{array}\right)\left(1-e^{-\left(\beta_{n}-F_{i}\right)}\right)^{n-i} & \leq\left(\frac{e n}{i}\right)^{2 i} \cdot\left(1-e^{-\left(\beta_{n}-F_{i}\right)}\right)^{n-i} \\
& \leq\left(\frac{e n}{i}\right)^{2 i} \cdot\left(1-e^{-\left(\beta_{n}-F_{i}\right)}\right)^{n-\zeta_{n}},
\end{aligned}
$$

where the last inequality follows since $1-e^{-\left(\beta_{n}-F_{i}\right)} \leq 1$ and $n-i \geq n-\zeta$. Combining the two results above yields the desired inequality.

We now have sufficient knowledge to prove Case 1 of Theorem 5.6

Proof of Theorem 5.6 (Case 1). Let $n$ be sufficiently large. Recall from Section 5.2.1 that $F_{\kappa} \leq \max \left\{2, f_{1}\right\}=O(1)$. Moreover, by our other observations in that section we also know that $\mathbb{E}[\mathrm{ALG}]=F_{\mathcal{K}}+\ln (n / \mathcal{K})+\Theta(1)=\ln (n / \mathcal{K})+\Theta(1)$. Set $\beta_{n}:=\ln (n / \kappa)(1+1 / n)^{-1}$ and observe that $\beta_{n}=\omega(1)$.

Conditioning on the events OPT $\geq \beta_{n}$ and OPT $<\beta_{n}$ yields

$$
\mathbb{E}\left[\frac{\mathrm{ALG}}{\mathrm{OPT}}\right] \leq \mathbb{E}\left[\frac{\mathrm{ALG}}{\beta_{n}}\right]+\mathbb{P}\left(\mathrm{OPT}<\beta_{n}\right) \cdot \mathbb{E}\left[\frac{\mathrm{ALG}}{\mathrm{OPT}} \mid \mathrm{OPT}<\beta_{n}\right]
$$

We start by bounding the second part, which can be rewritten and bounded as follows:

$$
\begin{aligned}
\mathbb{P}\left(\mathrm{OPT}<\beta_{n}\right) & \cdot \mathbb{E}\left[\frac{\mathrm{ALG}}{\mathrm{OPT}} \mid \mathrm{OPT}<\beta_{n}\right] \\
& =\mathbb{P}\left(\mathrm{OPT}<\beta_{n}\right) \cdot \int_{0}^{\infty} \mathbb{P}\left(\frac{\mathrm{ALG}}{\mathrm{OPT}} \geq x \mid \mathrm{OPT}<\beta_{n}\right) \mathrm{d} x \\
& \leq \mathbb{P}\left(\mathrm{OPT}<\beta_{n}\right) \cdot\left(\frac{1}{f_{1}^{2}}+\int_{1 / f_{1}^{2}}^{\infty} \mathbb{P}\left(\frac{\mathrm{ALG}}{\mathrm{OPT}} \geq x \mid \mathrm{OPT}<\beta_{n}\right) \mathrm{d} x\right) \\
& =\frac{1}{f_{1}^{2}} \cdot \mathbb{P}\left(\mathrm{OPT}>\beta_{n}\right)+\int_{1 / f_{1}^{2}}^{\infty} \mathbb{P}\left(\frac{\mathrm{ALG}}{\mathrm{OPT}} \geq x \text { and OPT }<\beta_{n}\right) \mathrm{d} x \\
& \leq \frac{1}{f_{1}^{2}} \cdot \mathbb{P}\left(\mathrm{OPT}>\beta_{n}\right)+\int_{1 / f_{1}^{2}}^{\infty} \mathbb{P}\left(\frac{\mathrm{ALG}}{\mathrm{OPT}} \geq x\right) \mathrm{d} x .
\end{aligned}
$$


Now observe that ALG/OPT $\geq x$ implies $A L G \geq \sqrt{x}$ or OPT $\leq 1 / \sqrt{x}$. This observation yields

$$
\begin{aligned}
& \mathbb{P}\left(\mathrm{OPT}<\beta_{n}\right) \cdot \mathbb{E}\left[\frac{\mathrm{ALG}}{\mathrm{OPT}} \mid \mathrm{OPT}<\beta_{n}\right] \\
& \leq \frac{1}{f_{1}^{2}} \cdot \mathbb{P}\left(\mathrm{OPT}>\beta_{n}\right)+\int_{1 / f_{1}^{2}}^{\infty} \mathbb{P}(\mathrm{ALG} \geq \sqrt{x}) \mathrm{d} x+\int_{1 / f_{1}^{2}}^{\infty} \mathbb{P}\left(\mathrm{OPT} \leq \frac{1}{\sqrt{x}}\right) \mathrm{d} x \\
& =\frac{1}{f_{1}^{2}} \cdot \mathbb{P}\left(\mathrm{OPT}>\beta_{n}\right)+\int_{1 / f_{1}^{2}}^{\infty} \mathbb{P}(\mathrm{ALG} \geq \sqrt{x}) \mathrm{d} x
\end{aligned}
$$

where the last equality follows since $\mathbb{P}\left(\mathrm{OPT} \leq f_{1}\right)=0$. The probability containing OPT can be bounded using Lemma 5.4. whereas the remaining integral can be bounded by Lemma 5.7 If we plug these bounds into our original upper bound for the expected approximation ratio, we obtain

$$
\mathbb{E}\left[\frac{\mathrm{ALG}}{\mathrm{OPT}}\right] \leq \mathbb{E}\left[\frac{\mathrm{ALG}}{\beta_{n}}\right]+\frac{1}{f_{1}^{2}} \sum_{i=1}^{\zeta_{n}}\left(\begin{array}{c}
n \\
i
\end{array}\right)\left(\begin{array}{c}
n-1 \\
i-1
\end{array}\right)\left(1-e^{-\left(\beta_{n}-F_{i}\right)}\right)^{n-i}+O\left(\frac{1}{n}\right)
$$

where $\zeta_{n}=\max \left\{k: \beta_{n} \geq F_{k}\right\}$. The terms of the summation can be bounded by Lemma 5.8 . This yields

$$
\mathbb{E}\left[\frac{\mathrm{ALG}}{\mathrm{OPT}}\right] \leq \mathbb{E}\left[\frac{\mathrm{ALG}}{\beta_{n}}\right]+\frac{1}{f_{1}^{2}} \sum_{i=1}^{\zeta_{n}} e^{-n}+O\left(\frac{1}{n}\right)=\mathbb{E}\left[\frac{\mathrm{ALG}}{\beta_{n}}\right]+O\left(\frac{1}{n}\right)
$$

since $\zeta_{n} \leq n$ by definition and since $1 / f_{1}^{2}$ is bounded by a polynomial in $n$. To see this, observe that $\kappa=o(n)$ implies $f_{n}>1 / n$ for sufficiently large $n$, and recall that we require that the ratios between facility opening costs are polynomially bounded, i.e., $f_{n} / f_{1} \leq n^{q}$ for some constant $q$ as $n \rightarrow \infty$. Finally, we use the values of $\mathbb{E}[A L G]$ and $\beta_{n}$ that we mentioned at the start of this proof to obtain that

$$
\begin{aligned}
\mathbb{E}\left[\frac{\mathrm{ALG}}{\mathrm{OPT}}\right] & \leq \frac{\mathbb{E}[\mathrm{ALG}]}{\beta_{n}}+O\left(\frac{1}{n}\right) \leq \frac{\ln (n / \kappa)+\Theta(1)}{\ln (n / \kappa)}\left(1+\frac{1}{n}\right)+O\left(\frac{1}{n}\right) \\
& =1+o(1),
\end{aligned}
$$

where the last equality follows since $k=o(n)$ implies $n / \kappa=\omega(1)$.

We are left with Case 2 of Theorem 5.6 Its proof requires four more lemmas. The first one allows us to bound the probability of the intersection of two dependent events.

Lemma 5.9. Let $A$ and $B$ be two arbitrary events. Then $\mathbb{P}(A \cap B) \leq \sqrt{\mathbb{P}(A) \mathbb{P}(B)}$. 
Proof. Let $X$ and $Y$ denote the indicator variables corresponding to $A$ and $B$, respectively. Then it follows that $\mathbb{E}[X]=\mathbb{E}\left[X^{2}\right]=\mathbb{P}(A)$ and $\mathbb{E}[Y]=\mathbb{E}\left[Y^{2}\right]=$ $\mathbb{P}(B)$. Moreover, we can see that $\mathbb{E}[X Y]=\mathbb{P}(A \cap B)$. Now, combining this knowledge with the Cauchy-Schwarz inequality for random variables, we derive

$$
\mathbb{P}(A \cap B)=\mathbb{E}[X Y] \leq \sqrt{\mathbb{E}\left[X^{2}\right] \mathbb{E}\left[Y^{2}\right]}=\sqrt{\mathbb{P}(A) \mathbb{P}(B)},
$$

which finishes this proof.

The other three lemmas that we need enable us to quickly bound certain expressions that show up in the proof of Case 2 of Theorem 5.6.

Lemma 5.10. Suppose that $F_{\kappa}=O(\ln (n))$ and set $m:=2 n-1$ to shorten notation. For sufficiently large $n$ we have

$$
F_{\kappa}+\int_{F_{\kappa}}^{\infty} \sqrt{1-\left(1-e^{-\left(x-F_{\kappa}\right)}\right)^{n-1}} \mathrm{~d} x \leq 2 \sqrt{e m}
$$

Proof. Let $n$ be sufficiently large. We start by applying the change of variables $y=x-F_{K}$ to the integral. This yields

$$
F_{\mathcal{K}}+\int_{F_{\mathcal{K}}}^{\infty} \sqrt{1-\left(1-e^{-\left(x-F_{\kappa}\right)}\right)^{n-1}} \mathrm{~d} x=F_{\mathcal{K}}+\int_{0}^{\infty} \sqrt{1-\left(1-e^{-y}\right)^{n-1}} \mathrm{~d} y .
$$

Next, we use Bernoulli's inequality to see that $\left(1-e^{-y}\right)^{n-1} \geq 1-(n-1) e^{-y}$ for all $y \in[0, \infty)$, from which we obtain that

$$
\begin{aligned}
F_{\mathcal{K}}+\int_{0}^{\infty} \sqrt{1-\left(1-e^{-y}\right)^{n-1}} \mathrm{~d} y & \leq F_{\mathcal{K}}+\int_{0}^{\infty} \sqrt{1-\left(1-(n-1) e^{-y}\right)} \mathrm{d} y \\
& =F_{\mathcal{K}}+\sqrt{n-1} \cdot \int_{0}^{\infty} e^{-y / 2} \mathrm{~d} y=F_{\kappa}+2 \sqrt{n-1} .
\end{aligned}
$$

Finally, since $F_{\mathcal{K}}=O(\ln (n))$, we have $F_{\kappa} \leq \sqrt{n-1}$ for $n$ sufficiently large. Combining this with the inequality $3<2 \sqrt{2}$, it follows that $F_{\kappa}+2 \sqrt{n-1} \leq$ $3 \sqrt{n-1} \leq 3 \sqrt{m} \leq 2 \sqrt{e m}$, which finishes this proof. 
Lemma 5.11. Let $\beta_{n}>0$ and $0<\zeta_{n} \leq n$, and set $m:=2 n-1$ to shorten notation. For sufficiently large $n$ we have

$$
\begin{gathered}
\frac{2 \sqrt{e m}}{f_{1}} \cdot \sqrt{\sum_{i=1}^{\zeta_{n}}\left(\begin{array}{c}
n \\
i
\end{array}\right)\left(\begin{array}{c}
n-1 \\
i-1
\end{array}\right)\left(1-e^{-\left(\beta_{n}-F_{i}\right)}\right)^{n-i}} \\
\quad \leq \frac{3}{f_{1}} \cdot(\mathrm{em})^{\zeta_{n}} \cdot e^{\beta_{n}} \cdot\left(1-e^{-\left(\beta_{n}-f_{1}\right)}\right)^{\frac{1}{2} n-\frac{1}{2} \zeta_{n}}
\end{gathered}
$$

Proof. We start by bounding the product of the binomials using the inequalities $\left(\begin{array}{l}n_{1} \\ k_{1}\end{array}\right)\left(\begin{array}{l}n_{2} \\ k_{2}\end{array}\right) \leq\left(\begin{array}{c}n_{1}+n_{2} \\ k_{1}+k+2\end{array}\right)$ and $\left(\begin{array}{l}n \\ k\end{array}\right) \leq(e n / k)^{k} \leq(e n)^{k}$ for $1 \leq k \leq n$. This results in

$$
\sum_{i=1}^{\zeta_{n}}\left(\begin{array}{l}
n \\
i
\end{array}\right)\left(\begin{array}{c}
n-1 \\
i-1
\end{array}\right)\left(1-e^{-\left(\beta_{n}-F_{i}\right)}\right)^{n-i} \leq \sum_{i=1}^{\zeta_{n}}(e m)^{2 i-1}\left(1-e^{-\left(\beta_{n}-F_{i}\right)}\right)^{n-1}
$$

Next, we use the Cauchy-Schwarz inequality for summations to obtain that

$$
\begin{aligned}
\sum_{i=1}^{\zeta_{n}}(e m)^{2 i-1}\left(1-e^{-\left(\beta_{n}-F_{i}\right)}\right)^{n-1} & \leq \sqrt{\sum_{i=1}^{\zeta_{n}}(e m)^{4 i-2} \cdot \sum_{i=1}^{\zeta_{n}}\left(1-e^{-\left(\beta_{n}-F_{i}\right)}\right)^{2 n-2 i}} \\
& \leq \sqrt{\sum_{i=1}^{\zeta_{n}}(e m)^{4 i-2} \cdot \sum_{i=1}^{\zeta_{n}}\left(1-e^{\left.-\left(\beta_{n}-f_{1}\right)\right)^{2 n-2 i}}\right.}
\end{aligned}
$$

where we also applied the inequality $F_{i} \geq f_{1}$. Now we can evaluate and bound both summations separately. This yields

$$
\sum_{i=1}^{\zeta_{n}}(\mathrm{em})^{4 i-2}=(\mathrm{em})^{2} \cdot \frac{(\mathrm{em})^{4 \zeta_{n}}-1}{(\mathrm{em})^{4}-1} \leq(\mathrm{em})^{2} \cdot \frac{(\mathrm{em})^{4 \zeta_{n}}}{(\mathrm{em})^{4}-1}
$$

and

$$
\begin{aligned}
\sum_{i=1}^{\zeta_{n}}\left(1-e^{-\left(\beta_{n}-f_{1}\right)}\right)^{2 n-2 i} & =\left(1-e^{-\left(\beta_{n}-f_{1}\right)}\right)^{2 n-2 \zeta_{n}} \cdot \frac{1-\left(1-e^{-\left(\beta_{n}-f_{1}\right)}\right)^{2 \zeta_{n}}}{1-\left(1-e^{-\left(\beta_{n}-f_{1}\right)}\right)^{2}} \\
& \leq \frac{\left(1-e^{-\left(\beta_{n}-f_{1}\right)}\right)^{2 n-2 \zeta_{n}}}{1-\left(1-e^{-\left(\beta_{n}-f_{1}\right)}\right)^{2}} \leq \frac{\left(1-e^{-\left(\beta_{n}-f_{1}\right)}\right)^{2 n-2 \zeta_{n}}}{e^{-\left(\beta_{n}-f_{1}\right)}}
\end{aligned}
$$


Combining the results above, and multiplying them with $4 \sqrt{\mathrm{em}} / f_{1}$, we get

$$
\begin{gathered}
\frac{2 \sqrt{e m}}{f_{1}} \cdot \sqrt{\sum_{i=1}^{\zeta_{n}}\left(\begin{array}{c}
n \\
i
\end{array}\right)\left(\begin{array}{c}
n-1 \\
i-1
\end{array}\right)\left(1-e^{\left.-\left(\beta_{n}-F_{i}\right)\right)^{n-i}}\right.} \\
\quad \leq \frac{2 \sqrt{e m}}{f_{1}} \cdot \sqrt[4]{(e m)^{2} \cdot \frac{(e m)^{4 \zeta_{n}}}{(e m)^{4}-1} \cdot \frac{\left(1-e^{\left.-\left(\beta_{n}-f_{1}\right)\right)^{2 n-2 \zeta_{n}}}\right.}{e^{-\left(\beta_{n}-f_{1}\right)}} .} .
\end{gathered}
$$

Next, upon rewriting and applying the inequalities $x /(x-1) \leq 2$ (for $x \geq 2$ ) and $2 \cdot \sqrt[4]{2}<3$, we obtain

$$
\begin{aligned}
\frac{2 \sqrt{e m}}{f_{1}} \cdot \sqrt{\sum_{i=1}^{\zeta_{n}}\left(\begin{array}{l}
n \\
i
\end{array}\right)\left(\begin{array}{c}
n-1 \\
i-1
\end{array}\right)\left(1-e^{\left.-\left(\beta_{n}-F_{i}\right)\right)^{n-i}}\right.} \\
\quad=\frac{2}{f_{1}} \cdot \sqrt[4]{\frac{(e m)^{4}}{(e m)^{4}-1} \cdot(e m)^{4 \zeta_{n}} \cdot \frac{\left(1-e^{\left.-\left(\beta_{n}-f_{1}\right)\right)^{2 n-2 \zeta_{n}}}\right.}{e^{-\left(\beta_{n}-f_{1}\right)}}} \\
\leq \frac{3}{f_{1}} \cdot \sqrt[4]{(e m)^{4 \zeta_{n}} \cdot \frac{\left(1-e^{-\left(\beta_{n}-f_{1}\right)}\right)^{2 n-2 \zeta_{n}}}{e^{-\left(\beta_{n}-f_{1}\right)}}} .
\end{aligned}
$$

Applying the inequalities $e^{-f_{1}} \leq 1$ and $e^{\beta_{n} / 4} \leq e^{\beta_{n}}$ yields the desired result.

Lemma 5.12. Let $\phi_{n}=f_{1} / \ln (n), \beta_{n}=\ln (n)\left(\phi_{n}+1\right)(1+1 / \ln \ln (n))^{-1}$ and $\zeta_{n}=\max \left\{k: \beta_{n} \geq F_{k}\right\}$. Moreover, set $m:=2 n-1$ to shorten notation and suppose that $f_{1}>1 / \ln ^{2}(n)$ as $n \rightarrow \infty$. For sufficiently large $n$ we have

$$
\frac{3}{f_{1}} \cdot(\mathrm{em})^{\zeta_{n}} \cdot e^{\beta_{n}} \cdot\left(1-e^{-\left(\beta_{n}-f_{1}\right)}\right)^{\frac{1}{2} n-\frac{1}{2} \zeta_{n}} \leq O\left(\frac{1}{n}\right) .
$$

Proof. First observe that, by definition of $\zeta_{n}$, we have $\zeta_{n} \leq \beta_{n} / f_{1}$, since $F_{k} \geq k f_{1}$ for all $k \in[n]$. We now show that the following inequality holds for sufficiently large $n$ :

$$
\zeta_{n} \ln (e m)+\beta_{n} \leq-2 \ln (n)+\left(\frac{1}{2} n-\frac{1}{2} \zeta_{n}\right) \cdot e^{f_{1}} \cdot e^{-\beta_{n}} .
$$

When analysing the left-hand side, we can see that $\zeta_{n} \leq \beta_{n} / f_{1}=O(\ln (n))$. $O\left(\ln ^{2}(n)\right)=O\left(\ln ^{3}(n)\right)$ and $\beta_{n}=O(\ln (n))$. So, the left-hand side is bounded by $O\left(\ln ^{4}(n)\right)$. When analysing the right-hand side, we can see that $\frac{1}{2} n-\frac{1}{2} \zeta_{n}=$ $\Omega(n)$, since $\zeta_{n} \leq O\left(\ln ^{3}(n)\right)$. Moreover, using the definitions of $\phi_{n}$ and $\beta_{n}$, we have $e^{f_{1}}=n^{\phi_{n}}$ and $e^{-\beta_{n}}=n^{\left(\phi_{n}+1\right)(-1+1 /(1+\ln \ln (n)))}$, where we used the equality $(1+1 / x)^{-1}=x /(1+x)=1-1 /(1+x)$ for $x=\ln \ln (n)$. From this we can deduce that $e^{f_{1}} \cdot e^{-\beta_{n}} \geq \Omega\left(n^{-1+1 /(1+\ln \ln (n))}\right)$. So, the right-hand side is bounded 
by $\Omega\left(n^{1 /(1+\ln \ln (n))}\right)$. Since $O\left(\ln ^{4}(n)\right)<\Omega\left(n^{1 /(1+\ln \ln (n))}\right)$, the stated inequality follows.

Now, rewriting the right-hand side and then applying the well-known inequality $1-x \leq-\ln (x)$ (for $x \geq 0$ ) yields

$$
\zeta_{n} \ln (e m)+\beta_{n} \leq-2 \ln (n)+\left(\frac{1}{2} \zeta_{n}-\frac{1}{2} n\right) \cdot \ln \left(1-e^{-\left(\beta_{n}-f_{1}\right)}\right) .
$$

Upon exponentiation of both sides we immediately obtain

$$
(e m)^{\zeta_{n}} \cdot e^{\beta_{n}} \leq \frac{1}{n^{2}} \cdot\left(1-e^{-\left(\beta_{n}-f_{1}\right)}\right)^{\frac{1}{2} \zeta_{n}-\frac{1}{2} n} .
$$

Using this inequality, in combination with $1 / f_{1} \leq \ln ^{2}(n)$ and $3 \ln ^{2}(n) / n^{2} \leq$ $O(1 / n)$, the desired result follows.

With these four lemmas, we are now ready to prove Case 2 of Theorem 5.6

Proof of Theorem 5.6 (Case 2). Recall that in Case 2 we have $f_{1}=O(\ln (n))$ and $f_{1}>1 / \ln ^{2}(n)$ as $n \rightarrow \infty$. Let $n$ be sufficiently large. Recall from Section 5.2.1 that $F_{\mathcal{K}} \leq \max \left\{2, f_{1}\right\}$. Moreover, by our other observations in that section we also know that $\mathbb{E}[\mathrm{ALG}]=F_{\kappa}+\ln (n / \kappa)+\Theta(1) \leq F_{\kappa}+\ln (n)+\Theta(1)$. Set $\phi_{n}:=$ $f_{1} / \ln (n)$ and $\beta_{n}:=\ln (n)\left(\phi_{n}+1\right)(1+1 / \ln \ln (n))^{-1}$, and observe that $\beta_{n}>F_{\mathcal{K}}$ for sufficiently large $n$.

Conditioning on the events OPT $\geq \beta_{n}$ and OPT $<\beta_{n}$ yields

$$
\mathbb{E}\left[\frac{\mathrm{ALG}}{\mathrm{OPT}}\right] \leq \mathbb{E}\left[\frac{\mathrm{ALG}}{\beta_{n}}\right]+\mathbb{P}\left(\mathrm{OPT}<\beta_{n}\right) \cdot \mathbb{E}\left[\frac{\mathrm{ALG}}{\mathrm{OPT}} \mid \mathrm{OPT}<\beta_{n}\right] .
$$

We start by bounding the second part. Since OPT $\geq f_{1}$ by definition, we may bound and subsequently rewrite the second part as follows:

$$
\begin{aligned}
\mathbb{P}\left(\mathrm{OPT}<\beta_{n}\right) \cdot \mathbb{E}\left[\frac{\mathrm{ALG}}{\mathrm{OPT}} \mid \mathrm{OPT}<\beta_{n}\right] & \leq \mathbb{P}\left(\mathrm{OPT}<\beta_{n}\right) \cdot \frac{\mathbb{E}\left[\mathrm{ALG} \mid \mathrm{OPT}<\beta_{n}\right]}{f_{1}} \\
& =\frac{1}{f_{1}} \cdot \int_{0}^{\infty} \mathbb{P}\left(\mathrm{ALG}>x \text { and OPT }<\beta_{n}\right) \mathrm{d} x .
\end{aligned}
$$

Since the events ALG $>x$ and OPT $<\beta_{n}$ are dependent, we use Lemma 5.9 to bound the probability inside the integral. This yields

$$
\begin{aligned}
\mathbb{P}\left(\mathrm{OPT}<\beta_{n}\right) \cdot \mathbb{E}\left[\frac{\mathrm{ALG}}{\mathrm{OPT}} \mid \mathrm{OPT}<\beta_{n}\right] & \\
\leq & \frac{1}{f_{1}} \cdot \sqrt{\mathbb{P}\left(\mathrm{OPT}<\beta_{n}\right)} \cdot \int_{0}^{\infty} \sqrt{\mathbb{P}(\mathrm{ALG}>x)} \mathrm{d} x .
\end{aligned}
$$


Now recall from Section 5.2.1 that we know the probability distribution of ALG, namely $\mathrm{ALG} \sim F_{\mathcal{K}}+\sum_{i=\kappa}^{n-1} \operatorname{Exp}(i)$. Let $\mathrm{UB} \sim F_{\mathcal{K}}+\sum_{i=1}^{n-1} \operatorname{Exp}(i)$, and observe that $\mathrm{ALG} \precsim \mathrm{UB}$. So, in particular it follows that $\mathbb{P}(\mathrm{ALG}>x) \leq \mathbb{P}(\mathrm{UB}>x)$ for all $x \in \mathbb{R}$. Moreover, from Lemma 3.4 we can derive that $\mathbb{P}(\mathrm{UB}>x)=1$ for $x<F_{\mathcal{K}}$ and $\mathbb{P}(\mathrm{UB}>x)=1-\left(1-e^{-\left(x-F_{\kappa}\right)}\right)^{n-1}$ for $x \geq F_{\kappa}$. Using these observations, it follows that

$$
\begin{aligned}
\int_{0}^{\infty} \sqrt{\mathbb{P}(\mathrm{ALG}>x)} \mathrm{d} x & \leq \int_{0}^{\infty} \sqrt{\mathbb{P}(\mathrm{UB}>x)} \mathrm{d} x \\
& =F_{\kappa}+\int_{F_{\kappa}}^{\infty} \sqrt{1-\left(1-e^{\left.-\left(x-F_{\kappa}\right)\right)^{n-1}}\right.} \mathrm{d} x \leq 2 \sqrt{e m},
\end{aligned}
$$

where we used Lemma 5.10 for the last inequality, and where we used $m:=$ $2 n-1$ to shorten notation. Using Lemma 5.4. we can bound the probability involving OPT as follows, where $\zeta_{n}:=\max \left\{k: \beta_{n} \geq F_{k}\right\}$ :

$$
\mathbb{P}\left(\mathrm{OPT}<\beta_{n}\right) \leq \sum_{i=1}^{\zeta_{n}}\left(\begin{array}{c}
n \\
i
\end{array}\right)\left(\begin{array}{c}
n-1 \\
i-1
\end{array}\right)\left(1-e^{-\left(\beta_{n}-F_{i}\right)}\right)^{n-i} .
$$

Combining all this information with the results of Lemmas 5.11 and 5.12 , we obtain that

$$
\begin{aligned}
\mathbb{P}\left(\mathrm{OPT}<\beta_{n}\right) \cdot \mathbb{E}\left[\frac{\mathrm{ALG}}{\mathrm{OPT}} \mid \mathrm{OPT}<\beta_{n}\right] & \leq \frac{3}{f_{1}} \cdot(e m)^{\zeta_{n}} e^{\beta_{n}}\left(1-e^{-\left(\beta_{n}-f_{1}\right)}\right)^{\frac{1}{2} n-\frac{1}{2} \zeta_{n}} \\
& \leq O\left(\frac{1}{n}\right) .
\end{aligned}
$$

Going back to our initial bound for the expected approximation ratio, we can now derive that

$$
\begin{aligned}
\mathbb{E}\left[\frac{\mathrm{ALG}}{\mathrm{OPT}}\right] & \leq \frac{\mathbb{E}[\mathrm{ALG}]}{\beta_{n}}+O\left(\frac{1}{n}\right) \\
& \leq \frac{F_{\kappa}+\ln (n)+\Theta(1)}{\beta_{n}}+O\left(\frac{1}{n}\right)=\frac{F_{\kappa}+\ln (n)}{\beta_{n}}+O\left(\frac{1}{\ln (n)}\right) .
\end{aligned}
$$

Now, if $f_{1}=o(\ln (n))$, then it follows that $\phi_{n}=f_{1} / \ln (n)=o(1)$ and $F_{\mathcal{K}}=$ $o(\ln (n))$. Hence, recalling that $\beta_{n}=\ln (n)\left(\phi_{n}+1\right)(1+1 / \ln \ln (n))^{-1}$, we obtain

$$
\begin{aligned}
\mathbb{E}\left[\frac{\mathrm{ALG}}{\mathrm{OPT}}\right] & \leq \frac{F_{\kappa}+\ln (n)}{\beta_{n}}+O\left(\frac{1}{\ln (n)}\right) \\
& =\frac{o(1)+1}{\phi_{n}+1} \cdot\left(1+\frac{1}{\ln \ln (n)}\right)+O\left(\frac{1}{\ln (n)}\right)=1+o(1) .
\end{aligned}
$$


On the other hand, if $f_{1}=\Theta(\ln (n))$, then it follows that $F_{\mathcal{K}}=f_{1}=\phi_{n} \ln (n)$, and hence we have

$$
\begin{aligned}
\mathbb{E}\left[\frac{\mathrm{ALG}}{\mathrm{OPT}}\right] & \leq \frac{F_{\kappa}+\ln (n)}{\beta_{n}}+O\left(\frac{1}{\ln (n)}\right)=\frac{\left(\phi_{n}+1\right) \ln (n)}{\beta_{n}}+\left(\frac{1}{\ln (n)}\right) \\
& =1+\frac{1}{\ln \ln (n)}+O\left(\frac{1}{\ln (n)}\right)=1+o(1),
\end{aligned}
$$

which finishes the proof of this case.

\subsubsection{Opening many facilities}

We continue with the next theorem, which provides a closed-form expression as an upper bound for the expected approximation ratio of our simple heuristic for $\kappa=\Theta(n)$.

Theorem 5.13. Define $\kappa:=\kappa\left(n ; f_{1}, \ldots, f_{n}\right)=\max \left\{i \in[n]: f_{i}<1 /(i-1)\right\}$. Let ALG denote the total cost of the solution that opens, independent of the metric space, the $\kappa$ cheapest facilities (breaking ties arbitrarily), i.e., the facilities with opening costs $f_{1}, \ldots, f_{\kappa}$. If $\kappa=\Theta(n)$, then for random shortest path metrics generated from complete graphs we have

$$
\begin{aligned}
& \mathbb{E}\left[\frac{\mathrm{ALG}}{\mathrm{OPT}}\right] \leq \sqrt{\max \left\{\frac{1}{F_{n}^{2}}, \max _{k \in[n-1]} \mathrm{O}\left(\frac{n^{4} F_{n-k}+k n^{2}}{n^{4} F_{n-k}^{3}+k^{4} F_{n-k}}\right)\right\}} \\
& +\sqrt[4]{O\left(\frac{n^{6} F_{n-1}^{2}+n^{2}}{n^{8} F_{n-1}^{7}+F_{n-1}^{3}}+\frac{n^{10} F_{n-2}^{3}+n^{4}}{n^{14} F_{n-2}^{9}+F_{n-2}^{2}}+\sum_{k=3}^{n-1} \frac{k^{3} n^{12} F_{n-k}^{3}+k^{9} n^{6}}{n^{16} F_{n-k}^{9}+k^{16} F_{n-k}}\right)} .
\end{aligned}
$$

Moreover, if $\kappa=n$, then for random shortest path metrics generated from complete graphs we have

$$
\begin{aligned}
& \mathbb{E}\left[\frac{\mathrm{ALG}}{\mathrm{OPT}}\right] \leq F_{n} \cdot \sqrt{\max \left\{\frac{1}{F_{n}^{2}}{ }^{\prime} \max _{k \in[n-1]} O\left(\frac{n^{4} F_{n-k}+k n^{2}}{n^{4} F_{n-k}^{3}+k^{4} F_{n-k}}\right)\right\}} \\
&+F_{n} \cdot \sqrt[4]{O\left(\frac{n^{6} F_{n-1}^{2}+n^{2}}{n^{8} F_{n-1}^{7}+F_{n-1}^{3}}+\frac{n^{10} F_{n-2}^{3}+n^{4}}{n^{14} F_{n-2}^{9}+F_{n-2}^{2}}+\sum_{k=3}^{n-1} \frac{k^{3} n^{12} F_{n-k}^{3}+k^{9} n^{6}}{n^{16} F_{n-k}^{9}+k^{16} F_{n-k}}\right)} .
\end{aligned}
$$

The proof of this theorem requires some bounds on expectations of probability distributions that are derived from an Erlang distribution. The results of the tedious computations that we used to obtain these bounds are stated in the following two lemmas. 
Lemma 5.14. Let $X_{k}=1 /\left(F_{n-k}+Z_{k}\right)^{2}$ where $Z_{k} \sim \sum_{i=1}^{k} \operatorname{Exp}\left(e\left(\begin{array}{l}n \\ 2\end{array}\right) / k\right)$. Then, for any $k \in[n-1]$ we have

$$
\mathbb{E}\left[X_{k}\right] \leq O\left(\frac{n^{4} F_{n-k}+k n^{2}}{n^{4} F_{n-k}^{3}+k^{4} F_{n-k}}\right)
$$

Proof. Let $h_{k}(x)$ denote the probability density function of $Z_{k}$, i.e.,

$$
h_{k}(x)=\left(\frac{e\left(\begin{array}{c}
n \\
2
\end{array}\right)}{k}\right)^{k} \cdot \frac{x^{k-1} e^{-e x\left(\begin{array}{c}
n \\
2
\end{array}\right) / k}}{(k-1) !} .
$$

For $k=1$ we obtain upon direct computation that

$$
\begin{aligned}
\mathbb{E}\left[X_{1}\right] & =\int_{0}^{\infty} \frac{h_{1}(x)}{\left(F_{n-1}+x\right)^{2}} \mathrm{~d} x=e\left(\begin{array}{c}
n \\
2
\end{array}\right) \cdot \int_{0}^{\infty} \frac{e^{-e x\left(\begin{array}{c}
n \\
2
\end{array}\right)}}{\left(F_{n-1}+x\right)^{2}} \mathrm{~d} x \\
& =e\left(\begin{array}{c}
n \\
2
\end{array}\right) \cdot\left(\frac{1}{F_{n-1}}-\frac{1}{F_{n-1}} \cdot e\left(\begin{array}{c}
n \\
2
\end{array}\right) F_{n-1} \cdot e^{e\left(\begin{array}{c}
n \\
2
\end{array}\right) F_{n-1}} \cdot \int_{e\left(\begin{array}{c}
n \\
2
\end{array}\right) F_{n-1}}^{\infty} \frac{e^{-t}}{t} \mathrm{~d} t\right) .
\end{aligned}
$$

Applying the second Padé approximant for the exponential integral in this expression (cf. 5.2 with $z=e\left(\begin{array}{c}n \\ 2\end{array}\right) F_{n-1}$ ), we obtain

$$
\begin{aligned}
\mathbb{E}\left[X_{1}\right] & \leq e\left(\begin{array}{c}
n \\
2
\end{array}\right)\left(\frac{1}{F_{n-1}}-\frac{1}{F_{n-1}} \cdot \frac{e^{2}\left(\begin{array}{c}
n \\
2
\end{array}\right)^{2} F_{n-1}^{2}+3 e\left(\begin{array}{c}
n \\
2
\end{array}\right) F_{n-1}}{e^{2}\left(\begin{array}{c}
n \\
2
\end{array}\right)^{2} F_{n-1}^{2}+4 e\left(\begin{array}{c}
n \\
2
\end{array}\right) F_{n-1}+2}\right) \\
& =\frac{e^{2}\left(\begin{array}{c}
n \\
2
\end{array}\right)^{2} F_{n-1}+2 e\left(\begin{array}{c}
n \\
2
\end{array}\right)}{e^{2}\left(\begin{array}{c}
n \\
2
\end{array}\right)^{2} F_{n-1}^{3}+4 e\left(\begin{array}{c}
n \\
2
\end{array}\right) F_{n-1}^{2}+2 F_{n-1}}=O\left(\frac{n^{4} F_{n-1}+n^{2}}{n^{4} F_{n-1}^{3}+F_{n-1}}\right),
\end{aligned}
$$

which satisfies the required bound.

For $k>1$ we obtain upon direct computation that

$$
\begin{aligned}
\mathbb{E}\left[X_{k}\right] & =\int_{0}^{\infty} \frac{h_{k}(x)}{\left(F_{n-1}+x\right)^{2}} \mathrm{~d} x=\frac{e^{k}\left(\begin{array}{c}
n \\
2
\end{array}\right)^{k}}{k^{k}(k-1) !} \cdot \int_{0}^{\infty} \frac{x^{k-1} e^{-e x\left(\begin{array}{c}
n \\
2
\end{array}\right) / k}}{\left(F_{n-k}+x\right)^{2}} \mathrm{~d} x \\
& =\frac{e^{2}\left(\begin{array}{l}
n \\
2
\end{array}\right)^{2}}{k^{2}(k-1)} \cdot\left(-1+\frac{k-1+z}{z} \cdot z^{k-1} e^{z} \int_{z}^{\infty} \frac{e^{-t}}{t^{k-1}} \mathrm{~d} t\right),
\end{aligned}
$$

where we used $z:=e\left(\begin{array}{c}n \\ 2\end{array}\right) F_{n-k} / k$ to shorten notation. Applying the second Padé approximant for the generalized exponential integral in this expression (cf. 5.5) 
with $\left.z=e\left(\begin{array}{l}n \\ 2\end{array}\right) F_{n-k} / k\right)$, we obtain

$$
\begin{aligned}
\mathbb{E}\left[X_{k}\right] & \leq \frac{e^{2}\left(\begin{array}{c}
n \\
2
\end{array}\right)^{2}}{k^{2}(k-1)} \cdot\left(-1+\frac{k-1+z}{z} \cdot \frac{z^{2}+(k+3) z+2}{z^{2}+2(k+1) z+k(k+1)}\right) \\
& =\frac{e^{2}\left(\begin{array}{c}
n \\
2
\end{array}\right)^{2} F_{n-k}+2 k e\left(\begin{array}{c}
n \\
2
\end{array}\right)}{e^{2}\left(\begin{array}{c}
n \\
2
\end{array}\right){ }^{2} F_{n-k}^{3}+2 k(k+1) e\left(\begin{array}{c}
n \\
2
\end{array}\right) F_{n-k}^{2}+k^{3}(k+1) F_{n-k}} \\
& =O\left(\frac{n^{4} F_{n-k}+k n^{2}}{n^{4} F_{n-k}^{3}+k^{4} F_{n-k}}\right),
\end{aligned}
$$

which completes this proof.

Lemma 5.15. Let $X_{k}=1 /\left(F_{n-k}+Z_{k}\right)^{2}$ where $Z_{k} \sim \sum_{i=1}^{k} \operatorname{Exp}\left(e\left(\begin{array}{c}n \\ 2\end{array}\right) / k\right)$. Then, for any $k \in\{3, \ldots, n-1\}$ we have

$$
\mathbb{E}\left[X_{k}^{2}\right]-\left(\mathbb{E}\left[X_{k}\right]\right)^{2} \leq O\left(\frac{k^{3} n^{12} F_{n-k}^{3}+k^{9} n^{6}}{n^{16} F_{n-k}^{9}+k^{16} F_{n-k}}\right)
$$

whereas for $k=1$ and $k=2$ we have

$$
\begin{aligned}
& \mathbb{E}\left[X_{1}^{2}\right]-\left(\mathbb{E}\left[X_{1}\right]\right)^{2} \leq O\left(\frac{n^{6} F_{n-1}^{2}+n^{2}}{n^{8} F_{n-1}^{7}+F_{n-1}^{3}}\right), \\
& \mathbb{E}\left[X_{2}^{2}\right]-\left(\mathbb{E}\left[X_{2}\right]\right)^{2} \leq O\left(\frac{n^{10} F_{n-2}^{3}+n^{4}}{n^{14} F_{n-2}^{9}+F_{n-2}^{2}}\right) .
\end{aligned}
$$

Proof. Let $h_{k}(x)$ denote the probability density function of $Z_{k}$, i.e.,

$$
h_{k}(x)=\left(\frac{e\left(\begin{array}{c}
n \\
2
\end{array}\right)}{k}\right)^{k} \cdot \frac{x^{k-1} e^{-e x\left(\begin{array}{c}
n \\
2
\end{array}\right) / k}}{(k-1) !} \text {. }
$$

We start by providing lower bounds for $\mathbb{E}\left[X_{k}\right]$. For $k=1$, we can use an approach similar to the one in the proof of Lemma 5.14 to obtain

$$
\begin{aligned}
\mathbb{E}\left[X_{1}\right] & =e\left(\begin{array}{c}
n \\
2
\end{array}\right) \cdot\left(\frac{1}{F_{n-1}}-\frac{1}{F_{n-1}} \cdot e\left(\begin{array}{c}
n \\
2
\end{array}\right) F_{n-1} \cdot e^{e\left(\begin{array}{c}
n \\
2
\end{array}\right) F_{n-1}} \cdot \int_{e\left(\begin{array}{c}
n \\
2
\end{array}\right) F_{n-1}}^{\infty} \frac{e^{-t}}{t} \mathrm{~d} t\right) \\
& \geq e\left(\begin{array}{c}
n \\
2
\end{array}\right) \cdot\left(\frac{1}{F_{n-1}}-\frac{1}{F_{n-1}} \cdot \frac{e\left(\begin{array}{c}
n \\
2
\end{array}\right) F_{n-1}+1}{e\left(\begin{array}{c}
n \\
2
\end{array}\right) F_{n-1}+2}\right)=\frac{e\left(\begin{array}{c}
n \\
2
\end{array}\right)}{e\left(\begin{array}{c}
n \\
2
\end{array}\right) F_{n-1}^{2}+2 F_{n-1}},
\end{aligned}
$$

where the inequality follows from applying the first Padé approximant for the exponential integral in the expression (cf. 5.1) with $\left.z=e\left(\begin{array}{c}n \\ 2\end{array}\right) F_{n-1}\right)$. 
For $k>1$, we can also use an approach similar to the one in the proof of Lemma 5.14. Again using $z:=e\left(\begin{array}{c}n \\ 2\end{array}\right) F_{n-k} / k$ to shorten notation, we obtain

$$
\begin{aligned}
\mathbb{E}\left[X_{k}\right] & =\frac{e^{2}\left(\begin{array}{c}
n \\
2
\end{array}\right)^{2}}{k^{2}(k-1)} \cdot\left(-1+\frac{k-1+z}{z} \cdot z^{k-1} e^{z} \int_{z}^{\infty} \frac{e^{-t}}{t^{k-1}} \mathrm{~d} t\right) \\
& \geq \frac{e^{2}\left(\begin{array}{c}
n \\
2
\end{array}\right)^{2}}{k^{2}(k-1)} \cdot\left(-1+\frac{k-1+z}{z} \cdot \frac{z^{2}+(k+1) z}{z^{2}+2 k z+k(k-1)}\right) \\
& =\frac{e^{2}\left(\begin{array}{c}
n \\
2
\end{array}\right)^{2}}{e^{2}\left(\begin{array}{c}
n \\
2
\end{array}\right)^{2} F_{n-k}^{2}+2 k^{2} e\left(\begin{array}{c}
n \\
2
\end{array}\right) F_{n-k}+k^{3}(k-1)},
\end{aligned}
$$

where the inequality follows from applying the second Padé approximant for the generalized exponential integral in the expression (cf. (5.5), again with $z=$ $\left.e\left(\begin{array}{c}n \\ 2\end{array}\right) F_{n-k} / k\right)$.

Next, we need upper bounds for $\mathbb{E}\left[X_{k}^{2}\right]$. For $k=1$ we obtain upon direct computation that

$$
\begin{aligned}
\mathbb{E}\left[X_{1}^{2}\right] & =\int_{0}^{\infty} \frac{h_{1}(x)}{\left(F_{n-1}+x\right)^{4}} \mathrm{~d} x=e\left(\begin{array}{c}
n \\
2
\end{array}\right) \cdot \int_{0}^{\infty} \frac{e^{-e x\left(\begin{array}{c}
n \\
2
\end{array}\right)}}{\left(F_{n-1}+x\right)^{4}} \mathrm{~d} x \\
& =e\left(\begin{array}{c}
n \\
2
\end{array}\right) \cdot\left(\frac{2-z+z^{2}}{6 F_{n-1}^{3}}-\frac{z^{2}}{6 F_{n-1}^{3}} \cdot z e^{z} \int_{z}^{\infty} \frac{e^{-t}}{t} \mathrm{~d} t\right),
\end{aligned}
$$

where we used $z:=e\left(\begin{array}{c}n \\ 2\end{array}\right) F_{n-1}$ to shorten notation. Applying the second Padé approximant for the exponential integral in this expression (cf. 5.2) with $z=$ $\left.e\left(\begin{array}{c}n \\ 2\end{array}\right) F_{n-1}\right)$, we obtain

$$
\begin{aligned}
\mathbb{E}\left[X_{1}^{2}\right] & \leq e\left(\begin{array}{l}
n \\
2
\end{array}\right) \cdot\left(\frac{2-z+z^{2}}{6 F_{n-1}^{3}}-\frac{z^{2}}{6 F_{n-1}^{3}} \cdot \frac{z^{2}+3 z}{z^{2}+4 z+2}\right) \\
& =\frac{3 e^{2}\left(\begin{array}{c}
n \\
2
\end{array}\right)^{2} F_{n-1}+2 e\left(\begin{array}{c}
n \\
2
\end{array}\right)}{3 e^{2}\left(\begin{array}{c}
n \\
2
\end{array}\right)^{2} F_{n-1}^{5}+12 e\left(\begin{array}{c}
n \\
2
\end{array}\right) F_{n-1}^{4}+6 F_{n-1}^{3}} .
\end{aligned}
$$

For $k=2$ we obtain upon direct computation that

$$
\begin{aligned}
\mathbb{E}\left[X_{2}^{2}\right] & =\int_{0}^{\infty} \frac{h_{2}(x)}{\left(F_{n-2}+x\right)^{4}} \mathrm{~d} x=\frac{e^{2}\left(\begin{array}{c}
n \\
2
\end{array}\right)^{2}}{4} \cdot \int_{0}^{\infty} \frac{x e^{-e x\left(\begin{array}{l}
n \\
2
\end{array}\right) / 2}}{\left(F_{n-2}+x\right)^{4}} \mathrm{~d} x \\
& =\frac{e^{2}\left(\begin{array}{l}
n \\
2
\end{array}\right)^{2}}{4} \cdot\left(\frac{1-2 z+z^{2}}{6 F_{n-2}^{2}}+\frac{z^{2}+3 z}{6 F_{n-2}^{2}} \cdot z e^{z} \int_{z}^{\infty} \frac{e^{-t}}{t} \mathrm{~d} t\right),
\end{aligned}
$$


where we used $z:=e\left(\begin{array}{c}n \\ 2\end{array}\right) F_{n-2} / 2$ to shorten notation. Applying the third Padé approximant for the exponential integral in this expression (cf. (5.3) with $z=$ $\left.e\left(\begin{array}{c}n \\ 2\end{array}\right) F_{n-2} / 2\right)$, we obtain

$$
\begin{aligned}
\mathbb{E}\left[X_{2}^{2}\right] & \leq \frac{e^{2}\left(\begin{array}{c}
n \\
2
\end{array}\right)^{2}}{4} \cdot\left(\frac{1-2 z+z^{2}}{6 F_{n-2}^{2}}+\frac{z^{2}+3 z}{6 F_{n-2}^{2}} \cdot \frac{z^{3}+11 z^{2}+26 z+6}{z^{3}+12 z^{2}+36 z+24}\right) \\
& =\frac{e^{3}\left(\begin{array}{c}
n \\
2
\end{array}\right)^{3} F_{n-2}+8 e^{2}\left(\begin{array}{c}
n \\
2
\end{array}\right)^{2}}{e^{3}\left(\begin{array}{c}
n \\
2
\end{array}\right)^{3} F_{n-2}^{5}+24 e^{2}\left(\begin{array}{c}
n \\
2
\end{array}\right)^{2} F_{n-2}^{4}+144 e\left(\begin{array}{c}
n \\
2
\end{array}\right) F_{n-2}^{3}+192 F_{n-2}^{2}} .
\end{aligned}
$$

For $k=3$ we obtain upon direct computation that

$$
\begin{aligned}
\mathbb{E}\left[X_{3}^{2}\right] & =\int_{0}^{\infty} \frac{h_{3}(x)}{\left(F_{n-3}+x\right)^{4}} \mathrm{~d} x=\frac{e^{3}\left(\begin{array}{c}
n \\
2
\end{array}\right)^{3}}{54} \cdot \int_{0}^{\infty} \frac{x^{2} e^{-e x\left(\begin{array}{c}
n \\
2
\end{array}\right) / 3}}{\left(F_{n-3}+x\right)^{4}} \mathrm{~d} x \\
& =\frac{e^{3}\left(\begin{array}{l}
n \\
2
\end{array}\right)^{3}}{54} \cdot\left(\frac{2+5 z+z^{2}}{6 F_{n-3}}-\frac{z^{2}+6 z+6}{6 F_{n-3}} \cdot z e^{z} \int_{z}^{\infty} \frac{e^{-t}}{t} \mathrm{~d} t\right),
\end{aligned}
$$

where we used $z:=e\left(\begin{array}{c}n \\ 2\end{array}\right) F_{n-3} / 3$ to shorten notation. Applying the fourth Padé approximant for the exponential integral in this expression (cf. (5.4) with $z=$ $\left.e\left(\begin{array}{c}n \\ 2\end{array}\right) F_{n-3} / 3\right)$, we obtain

$$
\begin{gathered}
\mathbb{E}\left[X_{3}^{2}\right] \leq \frac{e^{3}\left(\begin{array}{c}
n \\
2
\end{array}\right)^{3}}{54} \cdot\left(\frac{2+5 z+z^{2}}{6 F_{n-3}}-\frac{z^{2}+6 z+6}{6 F_{n-3}} \cdot \frac{z^{4}+15 z^{3}+58 z^{2}+50 z}{z^{4}+16 z^{3}+72 z^{2}+96 z+24}\right) \\
=\frac{e^{4}\left(\begin{array}{c}
n \\
2
\end{array}\right) F_{n-3}^{4}+12 e^{3}\left(\begin{array}{c}
n \\
2
\end{array}\right)^{3}}{e^{4}\left(\begin{array}{c}
n \\
2
\end{array}\right)^{4} F_{n-3}^{5}+48 e^{3}\left(\begin{array}{c}
n \\
2
\end{array}\right)^{3} F_{n-3}^{4}+648 e^{2}\left(\begin{array}{c}
n \\
2
\end{array}\right)^{2} F_{n-3}^{3}+2592 e\left(\begin{array}{c}
n \\
2
\end{array}\right) F_{n-3}^{2}+1944 F_{n-3}}
\end{gathered}
$$

For $k>3$ we obtain upon direct computation that

$$
\begin{aligned}
\mathbb{E}\left[X_{k}^{2}\right] & =\int_{0}^{\infty} \frac{h_{k}(x)}{\left(F_{n-k}+x\right)^{4}} \mathrm{~d} x=\frac{e^{k}\left(\begin{array}{c}
n \\
2
\end{array}\right)^{k}}{k^{k}(k-1) !} \cdot \int_{0}^{\infty} \frac{x^{k-1} e^{-e x\left(\begin{array}{c}
n \\
2
\end{array}\right) / k}}{\left(F_{n-k}+x\right)^{4}} \mathrm{~d} x \\
& =\frac{e^{4}\left(\begin{array}{c}
n \\
2
\end{array}\right)}{6 k^{4}(k-1)(k-2)(k-3)} \cdot\left(2 k-3-(z+k)^{2}+\right. \\
& \left.\frac{z^{3}+3(k-1) z^{2}+3(k-1)(k-2) z+(k-1)(k-2)(k-3)}{z} \cdot z^{k-3} e^{z} \int_{z}^{\infty} \frac{e^{-t}}{t^{k-3}} \mathrm{~d} t\right),
\end{aligned}
$$

where we used $z:=e\left(\begin{array}{c}n \\ 2\end{array}\right) F_{n-k} / k$ to shorten notation. After applying the fourth Padé approximant for the generalized exponential integral in this expression 
(cf. (5.6) with $z=e\left(\begin{array}{l}n \\ 2\end{array}\right) F_{n-k} / k$ ) and immediately simplifying the result, we obtain

$$
\begin{aligned}
\mathbb{E}\left[X_{k}^{2}\right] \leq & \left(e^{4}\left(\begin{array}{c}
n \\
2
\end{array}\right)^{4} F_{n-k}+4 k e^{3}\left(\begin{array}{c}
n \\
2
\end{array}\right)^{3}\right) /\left(e^{4}\left(\begin{array}{c}
n \\
2
\end{array}\right){ }^{4} F_{n-k}^{5}+4 k(k+1) e^{3}\left(\begin{array}{c}
n \\
2
\end{array}\right)^{3} F_{n-k}^{4}\right. \\
& \left.+6 k^{3}(k+1) e^{2}\left(\begin{array}{c}
n \\
2
\end{array}\right)^{2} F_{n-k}^{3}+4 k^{4}\left(k^{2}-1\right) e\left(\begin{array}{c}
n \\
2
\end{array}\right) F_{n-k}^{2}+k^{5}\left(k^{2}-1\right)(k-2) F_{n-k}\right) .
\end{aligned}
$$

Now observe that the bounds that we computed for $k=2$ and $k=3$ are actually the same as this bound. So, from now on we can use this last bound for any integer $k>1$.

It remains to combine the bounds that we have derived so far. For $k=1$ this yields

$$
\begin{aligned}
\mathbb{E}\left[X_{1}^{2}\right]- & \left(\mathbb{E}\left[X_{1}\right]\right)^{2} \leq \frac{3 e^{2}\left(\begin{array}{c}
n \\
2
\end{array}\right)^{2} F_{n-1}+2 e\left(\begin{array}{c}
n \\
2
\end{array}\right)}{3 e^{2}\left(\begin{array}{c}
n \\
2
\end{array}\right)^{2} F_{n-1}^{5}+12 e\left(\begin{array}{c}
n \\
2
\end{array}\right) F_{n-1}^{4}+6 F_{n-1}^{3}}-\left(\frac{e\left(\begin{array}{c}
n \\
2
\end{array}\right)}{e\left(\begin{array}{c}
n \\
2
\end{array}\right) F_{n-1}^{2}+2 F_{n-1}}\right)^{2} \\
& =\frac{2 e^{3}\left(\begin{array}{c}
n \\
2
\end{array}\right)^{3} F_{n-1}^{2}+14 e^{2}\left(\begin{array}{c}
n \\
2
\end{array}\right)^{2} F_{n-1}+8 e\left(\begin{array}{c}
n \\
2
\end{array}\right)}{3 e^{4}\left(\begin{array}{c}
n \\
2
\end{array}\right){ }^{4} F_{n-1}^{7}+24 e^{3}\left(\begin{array}{c}
n \\
2
\end{array}\right)^{3} F_{n-1}^{6}+66 e^{2}\left(\begin{array}{c}
n \\
2
\end{array}\right)^{2} F_{n-1}^{5}+72 e\left(\begin{array}{c}
n \\
2
\end{array}\right) F_{n-1}^{4}+24 F_{n-1}^{3}} \\
& =O\left(\frac{n^{6} F_{n-1}^{2}+n^{2}}{n^{8} F_{n-1}^{7}+F_{n-1}^{3}}\right) .
\end{aligned}
$$

For $k>1$ we obtain

$$
\begin{aligned}
\mathbb{E}\left[X_{k}^{2}\right] & -\left(\mathbb{E}\left[X_{k}\right]\right)^{2} \\
\leq & \left(e^{4}\left(\begin{array}{c}
n \\
2
\end{array}\right)^{4} F_{n-k}+4 k e^{3}\left(\begin{array}{c}
n \\
2
\end{array}\right)^{3}\right) /\left(e^{4}\left(\begin{array}{c}
n \\
2
\end{array}\right)^{4} F_{n-k}^{5}+4 k(k+1) e^{3}\left(\begin{array}{c}
n \\
2
\end{array}\right)^{3} F_{n-k}^{4}\right. \\
& \left.+6 k^{3}(k+1) e^{2}\left(\begin{array}{c}
n \\
2
\end{array}\right)^{2} F_{n-k}^{3}+4 k^{4}\left(k^{2}-1\right) e\left(\begin{array}{c}
n \\
2
\end{array}\right) F_{n-k}^{2}+k^{5}\left(k^{2}-1\right)(k-2) F_{n-k}\right) \\
& -\left(\frac{e^{2}\left(\begin{array}{c}
n \\
2
\end{array}\right)^{2}}{e^{2}\left(\begin{array}{c}
n \\
2
\end{array}\right)^{2} F_{n-k}^{2}+2 k^{2} e\left(\begin{array}{c}
n \\
2
\end{array}\right) F_{n-k}+k^{3}(k-1)}\right)^{2} \\
= & \frac{8 k^{3} e^{6}\left(\begin{array}{c}
n \\
2
\end{array}\right)^{6} F_{n-k}^{3}+(\ldots)+4 k^{7}(k-2)^{2} e^{3}\left(\begin{array}{c}
n \\
2
\end{array}\right)^{3}}{e^{8}\left(\begin{array}{c}
n \\
2
\end{array}\right)^{8} F_{n-k}^{9}+(\ldots)+k^{11}(k-1)^{2}\left(k^{2}-1\right)(k-2) F_{n-k}} \\
= & O\left(\frac{k^{3} n^{12} F_{n-k}^{3}+k^{9} n^{6}}{n^{16} F_{n-k}^{9}+k^{16} F_{n-k}}\right),
\end{aligned}
$$

where the final step only is true for $k>2$. For $k=2$ the last term in the denominator vanishes, which leads to the following result:

$$
\mathbb{E}\left[X_{2}^{2}\right]-\left(\mathbb{E}\left[X_{k}\right]\right)^{2} \leq O\left(\frac{n^{12} F_{n-2}^{3}+n^{6}}{n^{16} F_{n-2}^{9}+n^{2} F_{n-2}^{2}}\right)=O\left(\frac{n^{10} F_{n-2}^{3}+n^{4}}{n^{14} F_{n-2}^{9}+F_{n-2}^{2}}\right),
$$


which finishes this proof.

Using the bounds from the two foregoing lemmas, we can now prove Theorem5.13

Proof of Theorem 5.13 Using the Cauchy-Schwarz inequality for random variables (see Section 5.3, we obtain

$$
\mathbb{E}\left[\frac{\mathrm{ALG}}{\mathrm{OPT}}\right] \leq \sqrt{\mathbb{E}\left[\mathrm{ALG}^{2}\right]} \cdot \sqrt{\mathbb{E}\left[\frac{1}{\mathrm{OPT}^{2}}\right]}
$$

Recall from Section 5.2.1 that we know the distribution of ALG. We can use this to evaluate and bound $\mathbb{E}\left[\mathrm{ALG}^{2}\right]$. If $\kappa<n$, then we obtain

$$
\mathbb{E}\left[\mathrm{ALG}^{2}\right]=\left(F_{\kappa}+H_{n-1}-H_{\kappa-1}\right)^{2}+\sum_{i=\kappa}^{n-1} \frac{1}{i^{2}}=\left(F_{\kappa}+\ln (n / \kappa)+\Theta(1)\right)^{2}+\sum_{i=\kappa}^{n-1} \frac{1}{i^{2}}
$$

which is $O(1)$ since $\kappa=\Theta(n)$ and $F_{\kappa} \leq \max \left\{2, f_{1}\right\}=2$ for such $\kappa$ (and sufficiently large $n)$. If $\kappa=n$, then we have $\bar{E}\left[\mathrm{ALG}^{2}\right]=F_{n}^{2}$.

It remains to bound $\mathbb{E}\left[1 / \mathrm{OPT}^{2}\right]$. We start by using the observation OPT $=$ $\min _{k \in[n]} \mathrm{OPT}_{k}$ and the result of Lemma 5.5. This yields

$$
\mathbb{E}\left[\frac{1}{\mathrm{OPT}^{2}}\right]=\mathbb{E}\left[\max _{k \in\{0, \ldots, n-1\}} \frac{1}{\mathrm{OPT}_{n-k}^{2}}\right] \leq \mathbb{E}\left[\max _{k \in\{0, \ldots, n-1\}} \frac{1}{\left(F_{n-k}+Z_{k}\right)^{2}}\right],
$$

where $Z_{k} \sim \sum_{i=1}^{k} \operatorname{Exp}\left(e\left(\begin{array}{c}n \\ 2\end{array}\right) / k\right)$. The next step is to use the result of Lemma 5.1 to get rid of the maximum operator in the expectation. This yields

$$
\begin{aligned}
\mathbb{E}\left[\frac{1}{\mathrm{OPT}^{2}}\right] & \leq \max _{k \in\{0, \ldots, n-1\}} \mathbb{E}\left[\frac{1}{\left(F_{n-k}+Z_{k}\right)^{2}}\right]+\sqrt{\frac{n-1}{n} \cdot \sum_{k=0}^{n-1} \operatorname{Var}\left(\frac{1}{\left(F_{n-k}+Z_{k}\right)^{2}}\right)} \\
& \leq \max _{k \in\{0, \ldots, n-1\}} \mathbb{E}\left[X_{k}\right]+\sqrt{\sum_{k=0}^{n-1}\left(\mathbb{E}\left[X_{k}^{2}\right]-\left(\mathbb{E}\left[X_{k}\right]\right)^{2}\right)},
\end{aligned}
$$

where we also used $X_{k}:=1 /\left(F_{n-k}+Z_{k}\right)^{2}$ to shorten notation, applied the difference formula for the variance, and used the inequality $(n-1) / n \leq 1$.

Since we know the distribution of $Z_{k}$, we can evaluate and subsequently bound the expectations of $X_{k}$ that occur in this last expression. For $k=0$ we have $Z_{0}=0$, and thus $\mathbb{E}\left[X_{0}\right]=1 / F_{n}^{2}$ and $\mathbb{E}\left[X_{0}^{2}\right]-\left(\mathbb{E}\left[X_{0}\right]\right)^{2}=0$. For $k=$ $1, \ldots, n-1$, Lemmas 5.14 and 5.15 provide the bounds that we need to obtain the desired result.

We end this section by evaluating the bound from Theorem 5.13 for the special case where all facility opening costs are equal, i.e., $f_{1}=\ldots=f_{n}=f$. 
Corollary 5.16. Assume that $f_{1}=\ldots=f_{n}=f$ and define $\kappa:=\kappa\left(n ; f_{1}, \ldots, f_{n}\right)=$ $\max \left\{i \in[n]: f_{i}<1 /(i-1)\right\}=\min \{\lceil 1 / f\rceil, n\}$. Let ALG denote the total cost of the solution that opens, independent of the metric space, $\kappa$ arbitrarily chosen facilities, e.g., the facilities $\{1, \ldots, \kappa\}$. If $\kappa=\Theta(n)$, then for random shortest path metrics generated from complete graphs we have

$$
\mathbb{E}\left[\frac{\mathrm{ALG}}{\mathrm{OPT}}\right]=\mathrm{O}\left(1+\sqrt[4]{\ln (n) n^{3} f^{3}}\right)
$$

which for $f=O(1 / n \sqrt[3]{\ln (n)})$ is equal to $O(1)$. Moreover, if $f=o\left(1 / n^{3}\right)$, then this approximation ratio becomes $1+o(1)$.

The final part of this result is rather trivial since $\kappa=n$ when the facility opening costs satisfy $f=o\left(1 / n^{3}\right)$, and since these costs are then with high probability significantly less than the cheapest possible connection costs, i.e., the length of the shortest 'edge' in the random metric space, which is exponentially distributed with parameter $\left(\begin{array}{l}n \\ 2\end{array}\right)$. Before we prove this corollary, we need two more lemmas. The first one shows that the 'maximum-term' in the bound for the expected approximation ratio is asymptotically bounded by $O\left(1 / F_{n}^{2}\right)$ for the special case where all facility opening costs are equal.

Lemma 5.17. Suppose that $f=O(1 / n)$. Then, for any $k \in[n-1]$ we have

$$
O\left(\frac{n^{4}(n-k) f+k n^{2}}{n^{4}(n-k)^{3} f^{3}+k^{4}(n-k) f}\right) \leq O\left(\frac{1}{n^{2} f^{2}}\right)
$$

Moreover, if $f=o\left(1 / n^{3}\right)$, then for any $k \in[n-1]$ we have

$$
O\left(\frac{n^{4}(n-k) f+k n^{2}}{n^{4}(n-k)^{3} f^{3}+k^{4}(n-k) f}\right) \leq o\left(\frac{1}{n^{2} f^{2}}\right)
$$

Proof. We consider two main intervals for $k: k \leq n / 2$ and $k \geq n / 2$.

If $k \leq n / 2$, then we have $n-k=\Theta(n)$, and therefore it follows that

$$
O\left(\frac{n^{4}(n-k) f+k n^{2}}{n^{4}(n-k)^{3} f^{3}+k^{4}(n-k) f}\right)=O\left(\frac{n^{5} f+k n^{2}}{n^{7} f^{3}+k^{4} n f}\right)=O\left(\frac{n}{f} \cdot \frac{n^{3} f+k}{n^{6} f^{2}+k^{4}}\right)
$$

in this case. Now, if $k \leq \sqrt{n^{3} f}$ this last expression reduces to $O\left(n^{4} f / n^{6} f^{3}\right)=$ $O\left(1 / n^{2} f^{2}\right)$. On the other hand, if we have $k \geq n^{3} f$, then this last expression reduces to $O\left(k n / k^{4} f\right)=O\left(n / k^{3} f\right) \leq O(n / k f) \leq O\left(n / n^{3} f^{2}\right)=O\left(1 / n^{2} f^{2}\right)$, where the first inequality follows since $k \geq 1$ and the second since $k \geq n^{3} f$. In the remaining case, where $\sqrt{n^{3} f} \leq k \leq n^{3} f$, the last expression reduces to $O\left(n^{4} f / k^{4} f\right)=O\left(n^{4} / k^{4}\right) \leq O\left(n^{4} / n^{6} f^{2}\right)=O\left(1 / n^{2} f^{2}\right)$, where the inequality follows since $k \geq \sqrt{n^{3} f}$.

Moreover, if additionally $f=o\left(1 / n^{3}\right)$, then it follows that $n^{3} f=o(1)$, and thus (since $k \geq 1)$ the expression reduces to $O\left(k n / k^{4} f\right)=O\left(n / k^{3} f\right) \leq$ 
$O(n / k f) \leq o\left(n / n^{3} f^{2}\right)=o\left(1 / n^{2} f^{2}\right)$, where the first inequality follows again since $k \geq 1$ and the second since $k=\omega\left(n^{3} f\right)$.

If $k \geq n / 2$, then we have $k=\Theta(n)$, and therefore it follows that

$O\left(\frac{n^{4}(n-k) f+k n^{2}}{n^{4}(n-k)^{3} f^{3}+k^{4}(n-k) f}\right)=O\left(\frac{n^{4} g f+n^{3}}{n^{4} g^{3} f^{3}+n^{4} g f}\right)=O\left(\frac{1}{n g f} \cdot \frac{n g f+1}{g^{2} f^{2}+1}\right)$

in this case, where $g:=n-k$. Now, if $g \leq 1 / n f$ this last expression reduces to $O(1 / n g f) \leq O(g / n f) \leq O\left(1 / n^{2} f^{2}\right)$, where the first inequality follows since $g \geq 1$ and the second since $g \leq 1 / n f$. On the other hand, if we have $g \geq 1 / n f$, then this last expression reduces to $O(n g f / n g f)=O(1) \leq O\left(1 / n^{2} f^{2}\right)$, where the inequality follows since we still have $f=O(1 / n)$.

Moreover, if additionally $f=o\left(1 / n^{3}\right)$, then it follows that $1 / n f=\omega\left(n^{2}\right)$, and thus (since $g<n)$ the expression reduces to $O(1 / n g f) \leq O(g / n f) \leq$ $o\left(1 / n^{2} f^{2}\right)$, where the first inequality follows again since $g \geq 1$ and the second since $g=o(1 / n f)$.

The second lemma that we need provides a bound for the 'summation-term' in the bound for the expected approximation ratio for the special case where all facility opening costs are equal.

Lemma 5.18. If $f=O(1 / n)$, then we have

$$
\begin{aligned}
O\left(\frac{n^{8} f^{2}+n^{2}}{n^{15} f^{7}+n^{3} f^{3}}\right. & \left.+\frac{n^{13} f^{3}+n^{4}}{n^{25} f^{9}+n^{2} f^{2}}+\sum_{k=3}^{n-1} \frac{k^{3} n^{12}(n-k)^{3} f^{3}+k^{9} n^{6}}{n^{16}(n-k)^{9} f^{9}+k^{16}(n-k) f}\right) \\
& \leq O\left(\frac{\ln (n)}{n f}+\frac{1}{n^{4} f^{4}}\right) .
\end{aligned}
$$

Moreover, if $f=o\left(1 / n^{3}\right)$, then this result can be improved to $O\left(1 / n f^{3}\right)$.

Proof. The first two terms of this summation (corresponding to $k=1$ and $k=2$ ) are slightly different are be considered at the end of this proof. For the remaining terms of the summation, we consider again the intervals $k \geq n / 2$ and $k \leq n / 2$.

If $k \geq n / 2$, then we have $k=\Theta(n)$, and therefore it follows that

$$
\begin{aligned}
O\left(\frac{k^{3} n^{12}(n-k)^{3} f^{3}+k^{9} n^{6}}{n^{16}(n-k)^{9} f^{9}+k^{16}(n-k) f}\right) & =O\left(\frac{n^{15}(n-k)^{3} f^{3}+n^{15}}{n^{16}(n-k)^{9} f^{9}+n^{16}(n-k) f}\right) \\
& =O\left(\frac{1}{n(n-k) f} \cdot \frac{(n-k)^{3} f^{3}+1}{(n-k)^{8} f^{8}+1}\right) \\
& =O\left(\frac{1}{n(n-k) f}\right),
\end{aligned}
$$


where the last equality follows since $f=O(1 / n)$ and $k \geq n / 2$ implies that $(n-k) f=O(1)$. We also have

$$
\sum_{k=n / 2}^{n-1} O\left(\frac{1}{n(n-k) f}\right)=\sum_{g=1}^{n / 2} O\left(\frac{1}{n g f}\right)=O\left(\frac{\ln (n)}{n f}\right) .
$$

If $k \leq n / 2$, then we have $n-k=\Theta(n)$, and therefore it follows that

$$
\begin{aligned}
O\left(\frac{k^{3} n^{12}(n-k)^{3} f^{3}+k^{9} n^{6}}{n^{16}(n-k)^{9} f^{9}+k^{16}(n-k) f}\right) & =O\left(\frac{k^{3} n^{15} f^{3}+k^{9} n^{6}}{n^{25} f^{9}+k^{16} n f}\right) \\
& =O\left(\frac{k^{3} n^{5}}{f} \cdot \frac{n^{9} f^{3}+k^{6}}{n^{24} f^{8}+k^{16}}\right)
\end{aligned}
$$

We split the set $\mathcal{A}:=\{3, \ldots,\lfloor n / 2\rfloor\}$ into two parts: $\mathcal{A}_{1}:=\left\{k \in \mathcal{A}: k^{2} \leq n^{3} f\right\}$ and $\mathcal{A}_{2}:=\left\{k \in \mathcal{A}: k^{2}>n^{3} f\right\}$. Note that $\mathcal{A}=\mathcal{A}_{1} \cup \mathcal{A}_{2}$ and that $\mathcal{A}_{1}=\varnothing$ for sufficiently large $n$ if $f=o\left(1 / n^{3}\right)$. If $k \in \mathcal{A}_{1}$ then the last expression becomes $O\left(k^{3} n^{14} f^{3} / n^{24} f^{9}\right)=O\left(k^{3} / n^{10} f^{6}\right)$. On the other hand, if $k \in \mathcal{A}_{2}$ then it becomes $O\left(k^{9} n^{5} / k^{16} f\right)=O\left(n^{5} / k^{7} f\right)$. Summing these values yields

$$
\sum_{k \in \mathcal{A}_{1}} O\left(\frac{k^{3}}{n^{10} f^{6}}\right)=O\left(\frac{n^{6} f^{2}}{n^{10} f^{6}}\right)=O\left(\frac{1}{n^{4} f^{4}}\right)
$$

and

$$
\sum_{k \in \mathcal{A}_{2}} O\left(\frac{n^{5}}{k^{7} f}\right)=O\left(\frac{n^{5}}{n^{6} f}\right)=O\left(\frac{1}{n f}\right) .
$$

Now only the cases $k=1$ and $k=2$ remain. For $k=2$ we have

$$
O\left(\frac{n^{11} f^{3}+n^{2}}{n^{21} f^{9}+f^{2}}\right)=O\left(\frac{n^{2}}{f^{2}} \cdot \frac{n^{9} f^{3}+1}{n^{21} f^{7}+1}\right)= \begin{cases}O\left(n^{2} / f^{2}\right) & \text { if } f=o\left(1 / n^{3}\right) \\ O\left(1 / n^{10} f^{6}\right) & \text { if } f=\Omega\left(1 / n^{3}\right)\end{cases}
$$

whereas for $k=1$ we have

$$
O\left(\frac{n^{6} f^{2}+1}{n^{13} f^{7}+n f^{3}}\right)=O\left(\frac{1}{n f^{3}} \cdot \frac{n^{6} f^{2}+1}{n^{12} f^{4}+1}\right)= \begin{cases}O\left(1 / n f^{3}\right) & \text { if } f=o\left(1 / n^{3}\right) \\ O\left(1 / n^{7} f^{5}\right) & \text { if } f=\Omega\left(1 / n^{3}\right)\end{cases}
$$

Combining everything, for $f=\Omega\left(1 / n^{3}\right)$ we obtain

$$
\begin{gathered}
O\left(\frac{n^{8} f^{2}+n^{2}}{n^{15} f^{7}+n^{3} f^{3}}+\frac{n^{13} f^{3}+n^{4}}{n^{25} f^{9}+n^{2} f^{2}}+\sum_{k=3}^{n-1} \frac{k^{3} n^{12}(n-k)^{3} f^{3}+k^{9} n^{6}}{n^{16}(n-k)^{9} f^{9}+k^{16}(n-k) f}\right) \\
=O\left(\frac{1}{n^{7} f^{5}}+\frac{1}{n^{10} f^{6}}+\frac{1}{n^{4} f^{4}}+\frac{1}{n f}+\frac{\ln (n)}{n f}\right)=O\left(\frac{1}{n^{4} f^{4}}+\frac{\ln (n)}{n f}\right),
\end{gathered}
$$


whereas for $f=o\left(1 / n^{3}\right)$ we obtain

$$
\begin{gathered}
O\left(\frac{n^{8} f^{2}+n^{2}}{n^{15} f^{7}+n^{3} f^{3}}+\frac{n^{13} f^{3}+n^{4}}{n^{25} f^{9}+n^{2} f^{2}}+\sum_{k=3}^{n-1} \frac{k^{3} n^{12}(n-k)^{3} f^{3}+k^{9} n^{6}}{n^{16}(n-k)^{9} f^{9}+k^{16}(n-k) f}\right) \\
=O\left(\frac{1}{n f^{3}}+\frac{n^{2}}{f^{2}}+0+\frac{1}{n f}+\frac{\ln (n)}{n f}\right)=O\left(\frac{1}{n f^{3}}\right),
\end{gathered}
$$

which finishes this proof.

Combining the bounds from the two foregoing lemmas with the result of Theorem 5.13, we can now prove Corollary 5.16.

Proof of Corollary 5.16 Observe that $\kappa=\Theta(n)$ and $\kappa=\min \{\lceil 1 / f\rceil, n\}$ implies that $f=O(1 / n)$. We use Theorem 5.13 and start by bounding the 'maximumterm' in its result. Lemma 5.17 shows that in our special case this 'maximumterm' is asymptotically bounded by its first element, i.e., we can bound it by $O\left(1 / F_{n}^{2}\right)=O\left(1 / n^{2} f^{2}\right)$. Moreover, if $f=o\left(1 / n^{3}\right)$, then for sufficiently large $n$ it follows that the 'maximum-term' is equal to $1 / F_{n}^{2}=1 / n^{2} f^{2}$.

The 'summation-term' in the result of Theorem 5.13 can be evaluated by using the result of Lemma 5.18. Combining everything, for $f=\Theta(1 / n)$ it follows that

$$
\mathbb{E}\left[\frac{\mathrm{ALG}}{\mathrm{OPT}}\right] \leq \sqrt{\mathrm{O}\left(\frac{1}{n^{2} f^{2}}\right)}+\sqrt[4]{\mathrm{O}\left(\frac{\ln (n)}{n f}+\frac{1}{n^{4} f^{4}}\right)}=O(\sqrt[4]{\ln (n)}) .
$$

If $f=o(1 / n)$, then for sufficiently large $n$ it follows that $\kappa=n$, and therefore

$$
\begin{aligned}
\mathbb{E}\left[\frac{\mathrm{ALG}}{\mathrm{OPT}}\right] & \leq n f \cdot \sqrt{O\left(\frac{1}{n^{2} f^{2}}\right)}+n f \cdot \sqrt[4]{O\left(\frac{\ln (n)}{n f}+\frac{1}{n^{4} f^{4}}\right)} \\
& =O\left(1+\sqrt[4]{\ln (n) n^{3} f^{3}}\right)
\end{aligned}
$$

where the last term in general is bounded by $O(\sqrt[4]{\ln (n)})$ (since $f=O(1 / n)$ ) and more specifically by $O(1)$ if $f=O(1 / n \sqrt[3]{\ln (n)})$. Finally, if $f=o\left(1 / n^{3}\right)$, then we obtain

$$
\mathbb{E}\left[\frac{\mathrm{ALG}}{\mathrm{OPT}}\right] \leq n f \cdot \sqrt{\frac{1}{n^{2} f^{2}}}+n f \cdot \sqrt[4]{\mathrm{O}\left(\frac{1}{n f^{3}}\right)}=1+O\left(\sqrt[4]{n^{3} f}\right)=1+o(1)
$$

which finishes this proof. 


\section{Chapter 6}

\section{Minor Results on RSPM from Complete Graphs}

We end this thesis with two somewhat smaller results that emerged during our research. Both results concern random shortest path metrics generated from complete graphs.

In the first section we consider the Steiner tree problem. We extend a known result to show that a simple heuristic for this problem is asymptotically optimal as long as the number of terminals is not too large.

In the second section we consider the 2-opt heuristic for the traveling salesman problem. We present the results of some numerical simulations. These results seem to imply that the expected approximation ratio of this heuristic on random shortest path metrics generated from complete graphs is $\Theta(\ln (n))$. 


\subsection{Steiner Tree}

The first result in this chapter concerns the Steiner tree problem on random shortest path metrics generated from complete graphs. We consider the simple heuristic for the Steiner tree problem that we introduced in Section 1.2.5. Recall that this heuristic computes a minimum spanning tree on the subspace induced by $R$, the set of required vertices, and returns it as a solution, completely disregarding the vertices in $V \backslash R$.

Let ALG denote the total distance of the Steiner tree computed by this simple heuristic, and let ST denote the total distance of an optimal Steiner tree. We show that the simple heuristic is asymptotically optimal for random shortest path metrics generated from complete graphs if $|R|=o(n)$.

Theorem 6.1. Let $\varepsilon>0$ and suppose that $|R|=o(n)$. For random shortest path metrics generated from complete graphs we have $\mathbb{E}\left[\frac{\mathrm{ALG}}{\mathrm{ST}}\right] \leq 1+\varepsilon+o(1)$.

Proof. This proof builds directly upon a result by Bollobás et al. [12] for the Steiner tree problem on instances with independent edge lengths (i.e., instances generated by drawing independently a random edge weight $w(e)$ from the standard exponential distribution for each edge $e$ of a complete graph). For this purpose, let $\mathrm{W}$ denote the total weight of an optimal Steiner tree with respect to the weights $w$. We claim that $\mathrm{W}=\mathrm{ST}$.

To see this, first let $T^{\prime}=\left(V^{\prime}, E^{\prime}\right)$ denote an optimal Steiner tree with respect to the shortest path distances $d(u, v)$, i.e., ST $=\sum_{\{u, v\} \in E^{\prime}} d(u, v)$. Now obtain the tree $T^{\prime \prime}=\left(V^{\prime \prime}, E^{\prime \prime}\right)$ from $T^{\prime}$ by replacing each 'edge' $\{u, v\} \in E^{\prime}$ with a shortest $u, v$-path (with respect to the weights $w(e)$ ). Observe that $R \subseteq V^{\prime} \subseteq V^{\prime \prime}$ and $\sum_{\{u, v\} \in E^{\prime \prime}} d(u, v)=\sum_{\{u, v\} \in E^{\prime}} d(u, v)=\sum_{e \in E^{\prime}} w(e)$ by construction. So, $T^{\prime \prime}$ is a solution to the Steiner tree problem with respect to the weights as well. Hence $\mathrm{W} \leq \sum_{e \in E^{\prime}} w(e)=\sum_{\{u, v\} \in E^{\prime}} d(u, v)=\mathrm{ST}$.

Conversely, let $T^{*}=\left(V^{*}, E^{*}\right)$ denote an optimal Steiner tree with respect to the weights $w(e)$, i.e., $\mathrm{W}=\sum_{\{u, v\} \in E^{*}} w(e)$. Observe that $T^{*}$ is also a (not necessarily optimal) Steiner tree with respect to the distances $d(u, v)$. Hence $\mathrm{ST} \leq \sum_{\{u, v\} \in E^{*}} d(u, v) \leq \sum_{e \in E^{*}} w(e)=\mathrm{W}$, where we used the fact that $d(u, v) \leq$ $w(e)$ for any edge $e=\{u, v\}$ by construction.

So, indeed we have $\mathrm{W}=\mathrm{ST}$. Next, consider the following procedure to construct a spanning tree on the subspace induced by $R=\left\{v_{1}, \ldots, v_{|R|}\right\}$. For $r \in[|R|]$, define $x_{r}:=\operatorname{argmin}\left\{d\left(v_{r+1}, v_{i}\right): i \in[r]\right\}$ and let $E_{r}=\left\{v_{r+1}, v_{x_{r}}\right\}$ and $M_{r}=d\left(v_{r+1}, v_{x_{r}}\right)$, i.e., $M_{r}$ is the shortest distance from $v_{r+1}$ to $\left\{v_{1}, \ldots, v_{r}\right\}$ and $E_{r}$ is an 'edge' with that distance. Now, $T=\left(R, \bigcup_{r=1}^{|R|-1} E_{r}\right)$ is a spanning tree of total distance $\mathrm{M}:=\sum_{r=1}^{|R|-1} M_{r}$. In particular we have $A L G \leq M$ since ALG is a minimum spanning tree on the subspace induced by $R$.

Set $\tilde{\varepsilon}:=\varepsilon /(2+\varepsilon)$ and observe that $\tilde{\varepsilon}>0$. Now we can see that

$$
(1-\tilde{\varepsilon}) \cdot \frac{k-1}{n} \ln \left(\frac{n}{k}\right) \leq \mathrm{W}=\mathrm{ST} \leq \mathrm{ALG} \leq \mathrm{M} \leq(1+\tilde{\varepsilon}) \cdot \frac{k-1}{n} \ln \left(\frac{n}{k}\right)
$$


holds with probability $1-o(1)$, where the first and last inequality are by Bollobás et al. [12, Cor. 6] and the remaining (in)equalities follow from our observations above. So, in particular we have

$$
\frac{\mathrm{ALG}}{\mathrm{ST}} \leq \frac{1+\tilde{\varepsilon}}{1-\tilde{\varepsilon}}=1+\varepsilon
$$

if (6.1) holds, by our choice of $\tilde{\varepsilon}$. Now, let $\mathcal{E}$ denote the event that (6.1) holds. It follows that

$$
\begin{aligned}
\mathbb{E}\left[\frac{\mathrm{ALG}}{\mathrm{ST}}\right] & \leq \mathbb{E}\left[\frac{\mathrm{ALG}}{\mathrm{ST}} \mid \mathcal{E}\right]+\mathbb{E}\left[\frac{\mathrm{ALG}}{\mathrm{ST}} \mid \overline{\mathcal{E}}\right] \cdot \mathbb{P}(\overline{\mathcal{E}}) \\
& \leq 1+\varepsilon+2 \cdot o(1)=1+\varepsilon+o(1),
\end{aligned}
$$

where we used the fact that the worst-case approximation ratio of our simple heuristic on metric instances is given by $2-2 /|R|<2[46]$.

\subsection{Numerical Analysis of 2-Opt}

The second result in this chapter concerns the 2-opt heuristic for the traveling salesman problem on random shortest path metrics generated from complete graphs. Recall from Section 1.2.2 that this heuristic starts with an initial solution and iteratively improves this solution by applying so-called 2-exchanges until no improvement is possible anymore by any 2-exchange. Also recall from that section that in this thesis we look at the worst possible outcome of the 2-opt heuristic for a given instance.

Bringmann et al. [15] remarked that it is (almost) trivial that the 2-opt heuristic has an expected approximation ratio of $O(\ln (n))$ for random shortest path metrics generated from complete graphs. This is based on the facts that the total distance of the optimal tour is $\Theta(1)$ with high probability and that $\Delta_{\max }=$ $O(\ln (n) / n)$ for random shortest path metrics generated from complete graphs. In fact, using this argument one can show that any solution to the traveling salesman problem has an expected approximation ratio of $O(\ln (n))$ for random shortest path metrics generated from complete graphs.

With this in mind it makes sense to ask the question whether this approximation ratio could be improved to $o(\ln (n))$ for the 2-opt heuristic, as Bringmann et al. did [15]. Despite numerous attempts, we have not been able to give a definitive answer to this question. However, we have done some numerical simulations that seem to suggest that the 2-opt heuristic in fact has an expected approximation ratio of $\Theta(\ln (n))$.

For our numerical simulations we first generated many different instances of random shortest path metrics generated from complete graphs, for different values of $n$, the number of vertices. Then we solved two mixed integer linear programs on these instances using the AIMMS software and CPLEX solver: one 
to find the optimal solution to the traveling salesman problem and another one to find the worst 2-optimal solution for the traveling salesman problem, i.e., the worst possible outcome of the 2-opt heuristic. Finally, we divided for each instance the solution values of both mixed integer linear programs in order to obtain the approximation ratio of the 2-opt heuristic on that instance. The number of experiments done for each value of $n$ ranges from a couple of thousands for smaller values of $n$ to only a few hundred for larger values $n$, since especially the solving times for finding the worst 2-optimal solution increase significantly as $n$ grows.

The aggregated results of these numerical experiments are presented in Figures 6.1 and 6.2 In Figure 6.1 we have plotted the average approximation ratio of our experiments together with the $95 \%$ confidence intervals. In Figure 6.2 we divided all values by $\ln (n)$.

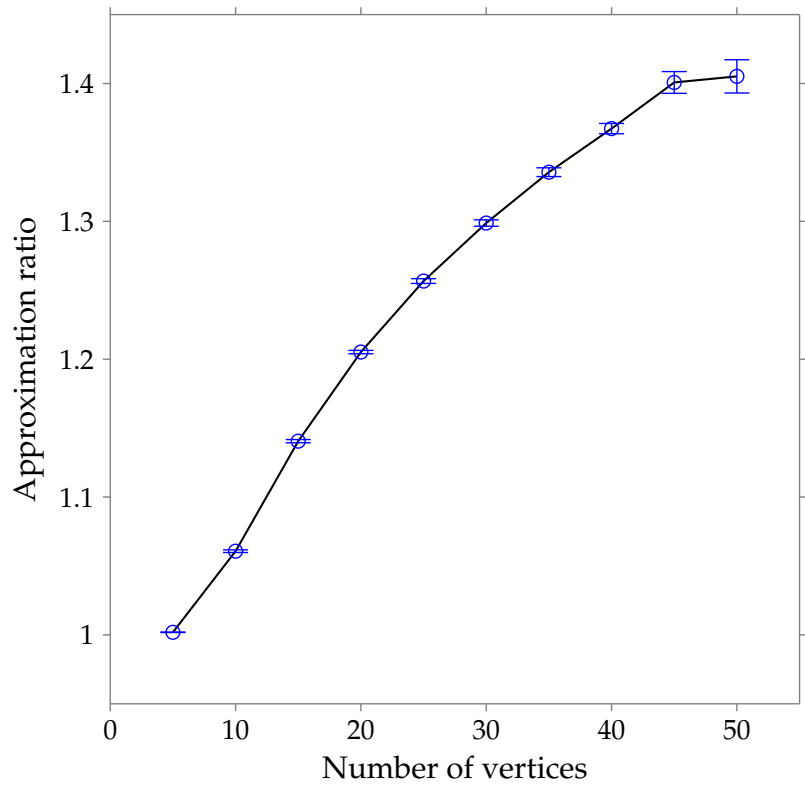

FIGURE 6.1: Simulation results for the expected approximation ratio of 2-opt on random shortest path metrics generated from complete graphs, with $95 \%$ confidence intervals.

Based on these figures, we conjecture that the approximation ratio for the 2-opt heuristic is probably $\Theta(\ln (n))$ for random shortest path metrics generated from complete graphs. Especially Figure 6.2 suggests that the scaled average approximation ratio seems to convert to some constant $c>0$. 


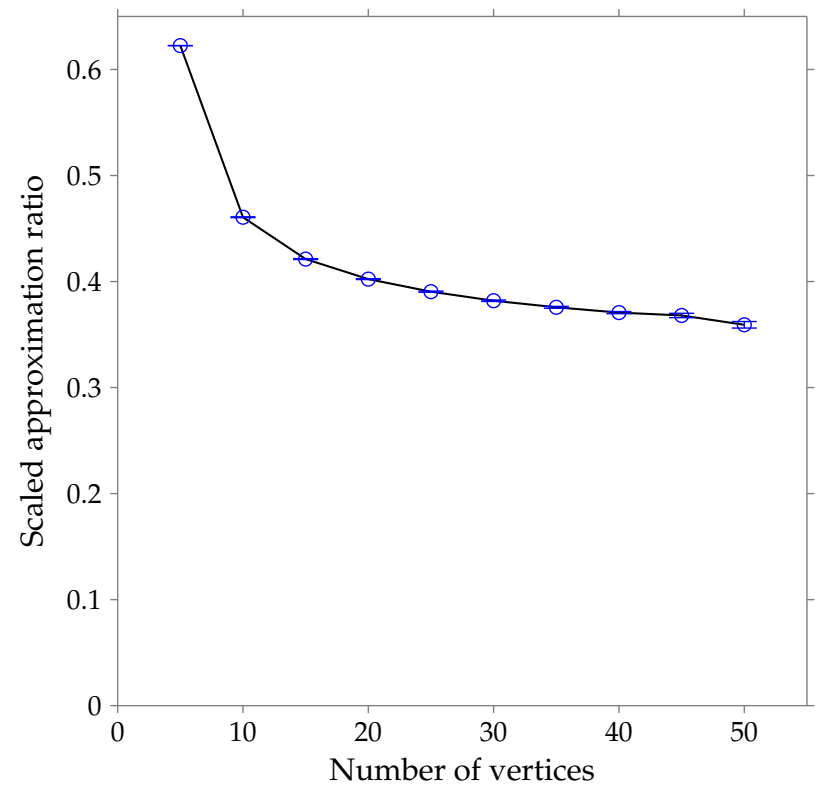

FIGURE 6.2: The same simulation results as in Figure 6.1 but now scaled by a factor of $\ln (n)$. 



\section{Chapter 7}

\section{Concluding Remarks}

In this thesis we presented several probabilistic analyses of some combinatorial optimizations on different variations of random shortest path metrics. By doing so we have started to widen the scope of models used for generating random instances of such combinatorial optimization problems beyond the currently used Euclidean models and models with independent edge lengths that both arguably have shortcomings regarding their realism for many applications.

From the variations of random shortest path metrics that we used in this thesis, we believe that random shortest path metrics generated from sparse graphs are the most realistic. However, it would have been rather difficult for us to perform the probabilistic analyses on this model without having researched the less realistic model of random shortest path metrics generated from complete (or dense Erdős-Rényi) graphs beforehand. Therefore, we think that it makes sense to also keep analysing heuristics for combinatorial optimization problems on these latter models.

In the remainder of this final chapter we focus a bit more on the usage of other distributions for generating random shortest path metrics, besides the exponential distribution, and on possibilities for future research. 


\subsection{Other Distributions}

As already briefly touched upon at the end of Chapter 3 in some cases our results can be generalized to random shortest path metrics generated using other probability distributions for the edge weights than the exponential distribution. In fact, the coupling argument that we used in Lemma 3.31 is a specific form of a more general coupling argument used by Janson [33] to show that results about the lengths of 'edges' in random shortest path metrics generated from complete graphs carry over if the exponential distribution is replaced by any probability distribution for which the cumulative distribution function $F(\cdot)$ satisfies $F(x)=x+o(x)$ as $x \rightarrow 0$. Observe that this property is, for instance, satisfied by the exponential distribution with parameter 1 (which we used in this thesis) and by the uniform distribution on the interval $[0,1]$.

As mentioned already in Section 3.5. intuitively this coupling argument follows from the fact that the 'edges' in the metric space have a length of $o(1)$ w.h.p., and hence only the behaviour of the edge weight distribution in a small interval $[0, o(1)]$ is relevant. The same intuitive argument holds for dense ErdősRényi random graphs as long as $p=\omega(\ln (n) / n)$ (cf. Lemma 3.32). Hence, we believe that all results in this thesis concerning random shortest path metrics generated from either complete graphs or Erdős-Rényi random graphs (with $p=\omega(\ln (n) / n))$ remain valid when using another probability distribution for which the cumulative distribution function $F(\cdot)$ satisfies $F(x)=x+o(x)$ as $x \rightarrow 0$.

For random shortest path metrics generated from sparse graphs, things are more complicated. In this model it becomes clear that the distribution of the distances uses significantly more information of the edge weight distribution than only a small interval of it. Hence, in general the results that we obtained using the exponential distribution do not directly carry over to other distributions in this case. Related to this problem, for random shortest path metrics generated from sparse random graphs Bhamidi et al. [11] showed that, under some mild conditions, the distribution of the distance between two arbitrarily chosen vertices behaves remarkably universal, irrespective of the distribution of the edge weights, as long as it is a continuous distribution. It is not immediately clear whether this result can be used to extend the results in this thesis related to random shortest path metrics generated from sparse graphs to other distributions for the edge weights, since those results consider the distances of more specific 'edges'.

\subsection{Future Work}

As mentioned in the previous section, for random shortest path metrics generated from sparse graphs the edge weight distribution has a significant influence on the distribution of the distances. In this thesis we used the exponential 
distribution, but for the 'realism aspect' it might be better to use some other distributions, for instance a uniform distribution.

Another potential way to improve the models of random shortest path metrics is to use only a (possibly random) subset of the vertices for defining the random metric space. In other words, after the 'regular' construction as described in Section 1.4 we restrict the distance function $d(\cdot, \cdot)$ to some sub-domain $V^{\prime} \times$ $V^{\prime}$, where $V^{\prime} \subset V$. Especially when considering random shortest path metrics generated from sparse graphs, this extension seems to improve the 'realism aspect' of the model since it resembles a situation where only a subset of the nodes in a (transportation) network is relevant for an optimization problem. It would be interesting to see whether this variation of the model could be analysed as well.

Finally, there are many other heuristics for the combinatorial optimization problems that we considered in this thesis, but also many other combinatorial optimization problems for which it would be interesting to know how they perform on random shortest path metrics. For instance, the Christofides algorithm for the traveling salesman problem or one of the polynomial time approximation algorithms for the facility location problem. Also, recall that in our analyses of the 2-opt heuristic for the traveling salesman problem we had to decouple the actual local search heuristic from the initialization in order to make our analyses tractable. It would be interesting to investigate hybrid heuristics that consist of an initialization combined with a local search procedure. 



\section{Bibliography}

[1] L. Addario-Berry, N. Broutin, and G. Lugosi. "The Longest MinimumWeight Path in a Complete Graph". In: Combinatorics, Probability and Computing 19.1 (2010), pp. 1-19. DOI: 10.1017/S0963548309990204.

[2] S. Ahn, C. Cooper, G. Cornuéjols, and A. Frieze. "Probabilistic Analysis of a Relaxation for the k-Median Problem". In: Mathematics of Operations Research 13.1 (1988), pp. 1-31. DOI: 10.1287/moor.13.1.1

[3] A. Auffinger, M. Damron, and J. Hanson. " 50 years of first passage percolation". In: arXiv e-prints, arXiv:1511.03262 (2015). arXiv: 1511.03262 [math.PR].

[4] T. Aven. "Upper (Lower) Bounds on the Mean of the Maximum (Minimum) of a Number of Random Variables". In: Journal of Applied Probability 22.3 (1985), pp. 723-728. DOI: 10.2307/3213876.

[5] D. Avis. "Two Greedy Heuristics for the Weighted Matching Problem". In: Congressus Numerantium XXI: Proceedings of the Ninth Southeastern Conference on Combinatorics, Graph Theory, and Computing, Florida Atlantic University, Boca Raton, January 30 - February 2, 1978. Ed. by F. Hoffman, D. McCarthy, R. C. Mullin, and R. G. Stanton. Winnipeg, Utilitas Mathematica Publishing Inc., 1978, pp. 65-76.

[6] D. Avis, B. Davis, and J. M. Steele. "Probabilistic Analysis of a Greedy Heuristic for Euclidean Matching". In: Probability in the Engineering and Informational Sciences 2.2 (1988), pp. 143-156. DOI: 10.1017/S0269964800 000711 .

[7] V. Bafna, B. Kalyanasundaram, and K. Pruhs. "Not all insertion methods yields constant approximate tours in the Euclidean plane". In: Theoretical Computer Science 125.2 (1994), pp. 345-353. DOI: 10.1016/0304-3975(94) 90257-7.

[8] J. L. Bentley and J. B. Saxe. "An Analysis of Two Heuristics for the Euclidean Traveling Salesman Problem". In: Proceedings of the Eighteenth Annual Allerton Conference on Communication, Control, and Computing, October 8-10, 1980, Allerton House, Monticello, Illinois. 1980, pp. 41-49.

[9] S. Bhamidi, R. van der Hofstad, and G. Hooghiemstra. "First passage percolation on Random Graphs with Finite Mean Degrees". In: The Annals of Applied Probability 20.5 (2010), pp. 1907-1965. DOI: 10.1214/09-AAP666. 
[10] S. Bhamidi, R. van der Hofstad, and G. Hooghiemstra. "First Passage Percolation on the Erdős-Rényi Random Graph". In: Combinatorics, Probability and Computing 20.5 (2011), pp. 683-707. DOI: 10.1017/S096354831100 023X.

[11] S. Bhamidi, R. van der Hofstad, and G. Hooghiemstra. "Universality for First Passage Percolation on Sparse Random Graphs". In: The Annals of Probability 45.4 (2017), pp. 2568-2630. DOI: 10.1214/16-AOP1120.

[12] B. Bollobás, D. Gamarnik, O. Riordan, and B. Sudakov. “On the Value of a Random Minimum Weight Steiner Tree". In: Combinatorica 24.2 (2004), pp. 187-207. DOI: 10.1007/s00493-004-0013-z.

[13] B. Bollobás and I. Leader. "Edge-Isoperimetric Inequalities in the Grid". In: Combinatorica 11.4 (1991), pp. 299-314. DOI: 10.1007/BF01275667

[14] J.-L. Bon and E. Păltănea. “Ordering Properties of Convolutions of Exponential Random Variables". In: Lifetime Data Analysis 5.2 (1999), pp. 185192. DOI: $10.1023 / \mathrm{A}: 1009605613222$.

[15] K. Bringmann, C. Engels, B. Manthey, and B. V. R. Rao. "Random Shortest Paths: Non-Euclidean Instances for Metric Optimization Problems". In: Algorithmica 73.1 (2015), pp. 42-62. DOI: 10.1007/s00453-014-9901-9

[16] J. Byrka, F. Grandoni, T. Rothvoss, and L. Sanità. "Steiner Tree Approximation via Iterative Randomized Rounding". In: Journal of the ACM 60.1 (2013), 6:1-6:33. DOI: 10.1145/2432622.2432628

[17] J. Byrka, T. Pensyl, B. Rybicki, A. Srinivasan, and K. Trinh. “An improved approximation for $k$-median, and positive correlation in budgeted optimization". In: Proceedings of the Twenty-Sixth Annual ACM-SIAM Symposium on Discrete Algorithms (SODA 2015). Ed. by P. Indyk. 2015, pp. 737756. DOI: $10.1137 / 1.9781611973730 .50$.

[18] B. Chandra, H. Karloff, and C. Tovey. "New Results on the Old k-opt Algorithm for the Traveling Salesman Problem". In: SIAM Journal on Computing 28.6 (1999), pp. 1998-2029. DOI: 10.1137/S0097539793251244

[19] G. Cornuejols, G. L. Nemhauser, and L. A. Wolsey. "The uncapacitated facility location problem". In: Discrete Location Theory. Ed. by P. B. Mirchandani and R. L. Francis. Wiley-Interscience, New York, 1990. Chap. 3, pp. 119-171. ISBN: 978-0-471-89233-5.

[20] R. Davis and A. Prieditis. "The expected length of a shortest path". In: Information Processing Letters 46.3 (1993), pp. 135-141. DOI: 10.1016/00200190(93)90059-I.

[21] M. E. Dyer and A. M. Frieze. “On patching algorithms for random asymmetric travelling salesman problems". In: Mathematical Programming 46 (1990), pp. 361-378. DOI: 10.1007/BF01585751 
[22] C. Engels and B. Manthey. "Average-case approximation ratio of the 2-opt algorithm for the TSP". In: Operations Research Letters 37.2 (2009), pp. 8384. DOI: $10.1016 / j$.orl.2008.12.002

[23] A. D. Flaxman, A. M. Frieze, and J. C. Vera. "On the Average Case Performance of Some Greedy Approximation Algorithms For the Uncapacitated Facility Location Problem". In: Combinatorics, Probability and Computing 16.5 (2007), pp. 713-732. DOI: 10.1017/S096354830600798X

[24] A. M. Frieze. "On the Value of a Random Minimim Spanning Tree Problem". In: Discrete Applied Mathematics 10.1 (1985), pp. 47-56. DOI: $10.1016 /$ 0166-218X (85) 90058-7.

[25] A. M. Frieze. "On Random Symmetric Travelling Salesman Problems". In: Mathematics of Operations Research 29.4 (2004), pp. 878-890. DOI:10.1287/ moor.1040.0105.

[26] A. M. Frieze and J. E. Yukich. "Probabilistic Analysis of the TSP". In: The Traveling Salesman Problem and Its Variations. Ed. by G. Gutin and A. P. Punnen. Springer, Boston, MA, 2007. Chap. 7, pp. 257-307. DOI: 10.1007/ 0-306-48213-4_7.

[27] J. M. Hammersley and D. J. A. Welsh. "First-Passage Percolation, Subadditive Processes, Stochastic Networks, and Generalized Renewal Theory". In: Bernoulli 1713 Bayes 1763 Laplace 1813, Anniversary Volume, Proceedings of an International Research Seminar Statistical Laboratory, University of California, Berkeley 1963. Ed. by J. Neyman and L. M. Le Cam. Springer Berlin Heidelberg, 1965, pp. 61-110. DOI: 10.1007/978-3-642-49750-6_7

[28] R. Hassin and E. Zemel. "On shortest paths in graphs with random weights". In: Mathematics of Operations Research 10.4 (1985), pp. 557-564. DOI: $10.1287 /$ moor.10.4.557.

[29] R. van der Hofstad, G. Hooghiemstra, and P. Van Mieghem. “First-passage percolation on the Random Graph". In: Probability in the Engineering and Informational Sciences 15.2 (2001), pp. 225-237. DOI: 10.1017/S0269964801 $15206 \mathrm{X}$.

[30] R. van der Hofstad, G. Hooghiemstra, and P. Van Mieghem. "Size and Weight of Shortest Path Trees with Exponential Link Weights". In: Combinatorics, Probability and Computing 15.6 (2006), pp. 903-926. DOI: 10.1017/ S0963548306007802.

[31] R. van der Hofstad, G. Hooghiemstra, and P. Van Mieghem. "The Weight of the Shortest Path Tree". In: Random Structures \& Algorithms 30.3 (2007), pp. 359-379. DOI: 10.1002/rsa.20141

[32] C. D. Howard. "Models of First-Passage Percolation". In: Probability on Discrete Structures. Ed. by H. Kesten. Springer Berlin Heidelberg, 2004, pp. 125-173. DOI: $10.1007 / 978-3-662-09444-0 \_3$. 
[33] S. Janson. "One, Two and Three Times $\log n / n$ for Paths in a Complete Graph with Random Weights". In: Combinatorics, Probability and Computing 8.4 (1999), pp. 347-361. DOI: 10.1017/S0963548399003892.

[34] S. Janson. "Tail bounds for sums of geometric and exponential variables". In: Statistics \& Probability Letters 135 (2018), pp. 1-6. DOI: $10.1016 / \mathrm{j} . \mathrm{spl}$. 2017.11 .017 .

[35] O. Kariv and S. L. Hakimi. "An Algorithmic Approach to Network Location Problems. II: The $p$-Medians". In: SIAM Journal on Applied Mathematics 37.3 (1979), pp. 539-560. DOI: 10.1137/0137041.

[36] R. M. Karp. "Reducibility among Combinatorial Problems". In: Complexity of Computer Problems. Ed. by R. E. Miller, J. W. Thatcher, and J. D. Bohlinger. The IBM Research Symposia Series. Boston, MA: Springer, 1972, pp. 85-103. DOI: 10.1007/978-1-4684-2001-2_9.

[37] R. M. Karp. "Probabilistic Analysis of Partitioning Algorithms for the Traveling-Salesman Problem in the Plane". In: Mathematics of Operations Research 2.3 (1977), pp. 209-224. DOI: $10.1287 /$ moor .2.3.209

[38] R. M. Karp. "A Patching Algorithm for the Nonsymmetric TravelingSalesman Problem". In: SIAM Journal on Computing 8.4 (1979), pp. 561573. DOI: $10.1137 / 0208045$.

[39] R. M. Karp and J. M. Steele. "Probabilistic Analysis of Heuristics". In: The Traveling Salesman Problem: A Guided Tour of Combinatorial Optimization. Ed. by E. L. Lawler, J. K. Lenstra, A. H. G. Rinnooy Kan, and D. B. Shmoys. John Wiley \& Sons Ltd., 1985, pp. 181-205. ISBN: 978-0-471-90413-7.

[40] S. Klootwijk and B. Manthey. "Probabilistic Analysis of Facility Location on Random Shortest Path Metrics". In: Computing with Foresight and Industry: Proceedings of the 15th Conference on Computability in Europe, CiE 2019, Durham, UK, July 15 - 19, 2019. Ed. by F. Manea, B. Martin, D. Paulusma, and G. Primiero. Lecture Notes in Computer Science 11558. Cham: Springer, 2019, pp. 37-49. DOI: 10.1007/978-3-030-22996-2_4.

[41] S. Klootwijk and B. Manthey. "Probabilistic Analysis of Optimization Problems on Sparse Random Shortest Path Metrics". In: Proceedings of the 31st International Conference on Probabilistic, Combinatorial and Asymptotic Methods for the Analysis of Algorithms (AofA 2020). Ed. by M. Drmota and C. Heuberger. Leibniz International Proceedings in Informatics (LIPIcs) 159. Dagstuhl, Germany: Schloss Dagstuhl - Leibniz-Zentrum für Informatik, 2020, 19:1-19:16. DOI: 10.4230/LIP Ics. Aof A . 2020.19.

[42] S. Klootwijk, B. Manthey, and S. K. Visser. "Probabilistic Analysis of Optimization Problems on Generalized Random Shortest Path Metrics". In: WALCOM: Algorithms and Computation, Proceedings of the 13th International Conference, WALCOM 2019, Guwahati, India, February 27 - March 2, 2019. 
Ed. by G. K. Das, P. S. Mandal, K. Mukhopadhyaya, and S. Nakano. Lecture Notes in Computer Science 11355. Cham: Springer, 2019, pp. 108-120. DOI: 10.1007/978-3-030-10564-8_9.

[43] S. Klootwijk, B. Manthey, and S. K. Visser. "Probabilistic analysis of optimization problems on generalized random shortest path metrics". In: Theoretical Computer Science 866 (2021), pp. 107-122. DOI: 10.1016/j.tcs . 2021.03 .016 .

[44] I. Kolossváry and J. Komjáthy. "First Passage Percolation on Inhomogeneous Random Graphs". In: Advances in Applied Probability 47.2 (2015), pp. 589-610. DOI: $10.1239 / \mathrm{aap} / 1435236989$

[45] B. Korte and J. Vygen. Combinatorial Optimization: Theory and Algorithms. 4th. Berlin, Heidelberg: Springer-Verlag, 2008. ISBN: 978-3-540-71843-7. DOI: $10.1007 / 978-3-540-71844-4$

[46] L. Kou, G. Markowsky, and L. Berman. "A Fast Algorithm for Steiner Trees". In: Acta Informatica 15 (1981), pp. 141-145. DOI: 10 . 1007 / BF002 88961.

[47] E. L. Lawler. Combinatorial Optimization: Networks and Matroids. New York: Holt, Rinehart and Winston, 1976. ISBN: 978-0-030-84866-7.

[48] S. Li. "A 1.488 approximation algorithm for the uncapacitated facility location problem". In: Information and Computation 222 (2013), pp. 45-58. DOI: 10.1016/j.ic.2012.01.007

[49] Y. L. Luke. "Chapter XIV Polynomial and Rational Approximations for the Incomplete Gamma Function". In: The Special Functions and their Approximations. Vol. 53, part 2. Mathematics in Science and Engineering. Elsevier, 1969, pp. 186-213. ISBN: 978-0-124-59902-4. DOI: 10.1016/S00765392(09)60074-6.

[50] M. Mahdian, Y. Ye, and J. Zhang. "Improved Approximation Algorithms for Metric Facility Location Problems". In: Approximation Algorithms for Combinatorial Optimization (APPROX 2002). Ed. by K. Jansen, S. Leonardi, and V. Vazirani. Lecture Notes in Computer Science 2462. Berlin, Heidelberg: Springer, 2002, pp. 229-242. DOI: 10.1007/3-540-45753-4_20

[51] M. Mitzenmacher and E. Upfal. Probability and Computing: Randomized Algorithms and Probabilistic Analysis. Cambridge, UK: Cambridge University Press, 2005. ISBN: 0-521-83540-2.

[52] H. N. Nagaraja. "Order Statistics from Independent Exponential Random Variables and the Sum of the Top Order Statistics". In: Advances in Distribution Theory, Order Statistics, and Inference. Ed. by N. Balakrishnan, J. M. Sarabia, and E. Castillo. Birkhäuser Boston, 2006. Chap. 11, pp. 173-185. DOI: $10.1007 / 0-8176-4487-3 \_11$. 
[53] G. Reinelt. The Traveling Salesman: Computational Solutions for TSP Applications. Lecture Notes in Computer Science 840. Berlin, Heidelberg: Springer-Verlag, 1994. ISBN: 978-3-540-58334-9. DOI: 10 . 1007 / 3 - $540-$ 48661-5.

[54] E. M. Reingold and R. E. Tarjan. "On a greedy heuristic for complete matching". In: SIAM Journal on Computing 10.4 (1981), pp. 676-681. DOI: $10.1137 / 0210050$

[55] A. Rényi. "On the theory of order statistics". In: Acta Mathematica Academiae Scientiarum Hungarica 4.3-4 (1953), pp. 191-231. DOI:10.1007/BF0 2127580.

[56] D. J. Rosenkrantz, R. E. Stearns, and P. M. Lewis II. “An Analysis of Several Heuristics for the Traveling Salesman Problem". In: SIAM Journal on Computing 6.3 (1977), pp. 563-581. DOI: 10.1137/0206041.

[57] S. M. Ross. Introduction to Probability Models. 10th. Burlington, MA: Academic Press, 2010. ISBN: 978-0-12-375686-2.

[58] J. J. A. Slootbeek. "Average-Case Analysis of the 2-opt Heuristic for the TSP". Master Thesis. University of Twente, 2017. URL: essay . utwente . nl/72060/

[59] D. W. Walkup. "On the Expected Value of a Random Assignment Problem". In: SIAM Journal on Computing 8.3 (1979), pp. 440-442. DOI: 10.1137/ 0208036 . 


\section{Summary}

Simple heuristics for combinatorial optimization problems often show a remarkable performance in practice. Worst-case analysis often falls short of explaining this performance. Because of this, 'beyond worst-case analysis' of algorithms has recently gained a lot of attention, including probabilistic analysis of algorithms.

The instances of many combinatorial optimization problems are essentially a discrete metric space. Probabilistic analysis for such metric optimization problems has nevertheless mostly been conducted on instances drawn from Euclidean space, which provides a structure that is usually heavily exploited in the analysis. However, most instances from practice are not Euclidean. Little work has been done on metric instances drawn from other, more realistic, distributions. Some initial results have been obtained by Bringmann et al. (Algorithmica, 2015), who have used random shortest path metrics generated from complete graphs to analyse heuristics.

In this thesis we look at several variations of the random shortest path metrics, and perform probabilistic analyses for some simple heuristics for several combinatorial optimization problems on these random metric spaces. A random shortest path metric is constructed by drawing independent random edge weights for each edge in a graph and setting the distance between every pair of vertices to the length of a shortest path between them, with respect to the drawn weights.

We provide some basic properties of the distances between vertices in random shortest path metrics. Using these properties, we perform several probabilistic analyses. For random shortest path metrics generated from (dense) Erdős-Rényi random graphs we show that the greedy heuristic for the minimum-distance perfect matching problem, the nearest neighbor and insertion heuristics for the traveling salesman problem, and a trivial heuristic for the $k$ median problem all achieve a constant expected approximation ratio. Additionally, we show a polynomial upper bound for the expected number of iterations of the 2-opt heuristic for the traveling salesman problem in this model.

For random shortest path metrics generated from sparse graphs we show that the greedy heuristic for the minimum-distance perfect matching problem, and the nearest neighbor and insertion heuristics for the traveling salesman problem all achieve a constant expected approximation ratio. Additionally, we show that the 2-opt heuristic for the traveling salesman problem also achieves a constant expected approximation ratio in this model. 
For random shortest path metrics generated from complete graphs we analyse a simple greedy heuristic for the facility location problem: opening the $\kappa$ cheapest facilities (with $\kappa$ only depending on the facility opening costs). If the facility opening costs are such that $\kappa$ is not too large, then we show that this heuristic is asymptotically optimal. For large values of $\kappa$ we provide a closedform expression as upper bound for the expected approximation ratio and we evaluate this expression for the special case where all facility opening costs are equal.

Moreover, we show in this model that a simple 2-approximation algorithm for the Steiner tree problem is asymptotically optimal as long as the number of terminals is not too large. We also present some numerical results that imply that the 2-opt heuristic for the traveling salesman problem seems to perform rather poorly in this model. 


\section{Samenvatting}

Eenvoudige heuristieken voor combinatorische optimalisatieproblemen geven vaak opmerkelijk goede resultaten in de praktijk. Worstcase-analyse schiet vaak tekort om dit gedrag te verklaren. Om deze reden heeft de zogeheten 'beyond worst-case'-analyse recentelijk veel belangstelling gekregen. Hieronder valt onder andere probabilistische analyse van algoritmes.

De probleemgevallen van veel combinatorische optimalisatieproblemen zijn in wezen een discrete metrische ruimte. Probabilistische analyses voor zulke metrische optimalisatieproblemen zijn desdondanks voornamelijk gedaan op probleemgevallen gebaseerd op Euclidische ruimten, die een structuur bieden waarvan in de analyse flink wordt gebruikgemaakt. Echter, de meeste probleemgevallen uit de praktijk zijn niet Euclidisch. Er is nog maar weinig onderzoek gedaan met metrische probleemgevallen gebaseerd op andere, realistischere, verdelingen. Bringmann et al. (Algorithmica, 2015) hebben een aantal initiële resultaten verkregen waarbij ze gebruikmaakten van toevallige kortstepadmetrieken gegenereerd vanuit complete grafen om heuristieken te analyseren.

In deze scriptie kijken we naar meerdere variaties van de toevallige kortstepadmetrieken en voeren we probabilistische analyses uit van een paar eenvoudige heuristieken voor een aantal combinatorische optimalisatieproblemen op deze toevallige kortstepadmetrieken. Een toevallige kortstepadmetriek wordt geconstrueerd door onafhankelijke toevallige lijngewichten te trekken voor elke lijn in een graaf en vervolgens de afstand tussen elk tweetal punten gelijk te stellen aan de lengte van het kortste pad tussen die twee punten, gemeten aan de hand van de getrokken gewichten.

We laten een paar basiseigenschappen zien van de afstanden tussen punten in toevallige kortstepadmetrieken. Gebruikmakend van deze eigenschappen voeren we een aantal probabilistische analyses uit. Voor toevallige kortstepadmetrieken gegenereerd vanuit (dichte) Erdós-Rényi-grafen laten we zien dat de gretige heuristiek voor het minimale-afstand-perfectekoppelings-probleem, de meest-nabije-buur- en insertieheuristieken voor het handelsreizigersprobleem, en een triviale heuristiek voor het $k$-mediaanprobleem allemaal een constante verwachte approximatieratio hebben. Bovendien laten we zien dat het verwachte aantal iteraties van de 2-opt-heuristiek voor het handelsreizigersprobleem polynomiaal begrensd is in dit model.

Voor toevallige kortstepadmetrieken gegenereerd vanuit ijle grafen laten we 
zien dat de gretige heuristiek voor het minimale-afstand-perfectekoppelingsprobleem, en de meest-nabije-buur- en insertieheuristieken voor het handelsreizigersprobleem allemaal een constante verwachte approximatieratio hebben. Bovendien laten we zien dat de 2-opt-heuristiek voor het handelsreizigersprobleem ook een constante approximatieratio heeft in dit model.

Voor toevallige kortstepadmetrieken gegenereerd vanuit complete grafen analyseren we een simpele gretige heuristiek voor een locatieprobleem. Deze heuristiek opent de $\kappa$ goedkoopste locaties (waarbij $\kappa$ alleen afhankelijk is van de openingskosten voor alle locaties). Als de openingskosten dusdanig zijn dat $\kappa$ niet te groot is, dan laten we zien dat deze heuristiek asymptotisch optimaal is. Voor grote waarden van $\kappa$ geven we een uitdrukking in gesloten vorm als bovengrens voor de verwachte approximatieratio en evalueren we deze uitdrukking in het speciale geval waarin de openingskosten voor elke locatie hetzelfde zijn.

Daarnaast laten we in dit model zien dat een eenvoudige 2-approximatie voor het Steinerboomprobleem asymptotisch optimaal is mits het aantal eindpunten niet te groot is. We geven ook een paar numerieke resultaten die impliceren dat de 2-opt-heuristiek voor het handelsreizigersprobleem tamelijk slecht lijkt te presteren in dit model. 


\section{Dankwoord}

Dit proefschrift was nooit tot stand gekomen zonder de hulp en steun van verschillende personen. Hierbij wil ik iedereen bedanken die op de een of andere manier, direct of indirect, heeft bijgedragen aan de voltooiing van mijn promotietraject. Een aantal van deze personen wil ik graag in het bijzonder bedanken.

Bodo, richting het eind van mijn eerste jaar als masterstudent aan de Universiteit Twente benaderde je mij met de vraag of ik interesse had om als PhDstudent onder jouw begeleiding aan de slag te gaan. Op het moment dat ik dat aanbod accepteerde kon ik nog niet voorzien wat de daaropvolgende jaren mij allemaal zouden brengen, maar terugkijkend kan ik zeggen dat ik voornamelijk heb genoten van de spreekwoordelijke achtbaanrit die ik heb gemaakt. Bedankt voor het geven van deze kans, en bedankt voor alle waardevolle begeleiding en feedback die je mij in de afgelopen jaren hebt gegeven, de eerste vier jaar als copromotor en het laatste jaar als mijn promotor.

Marc, bedankt dat je de eerste vier jaar mijn promotor bent geweest, ook al beperkte jouw aandeel in mijn begeleiding zich voornamelijk tot administratieve taken, naast de dagelijkse begeleiding die ik van Bodo kreeg. Fijn dat je nu deel uitmaakt mijn promotiecommissie.

Dan de rest van mijn promotiecommissie: Nelly, Remco, Ruben en Tjark, bedankt dat jullie onderdeel van deze commissie willen zijn, en bedankt voor de tijd en moeite die jullie hebben genomen om dit proefschrift te lezen, te beoordelen en van feedback te voorzien.

De afgelopen jaren heb ik deel uit mogen maken van de vakgroep DMMP, die de laatste jaren samen met de vakgroepen SOR en STAT het cluster MOR vormt. Ik heb de afgelopen jaren genoten van de fijne werksfeer die hier heerst. Veel dank aan mijn medepromovendi en (oud-)collega's van deze vakgroepen voor de gezellige momenten bij de koffiemachine (ook al drink ik zelf geen koffie), de leuke lunchpauzes (met of zonder wandeling), en alle andere leuke en gezellige momenten die hier niet onder vallen.

Tot slot wil ik mijn familie en vrienden bedanken voor hun steun. Papa en Mama, bedankt voor de interesse die jullie keer op keer in mijn onderzoek hebben getoond, ook al kon ik niet altijd even goed uitleggen waar ik nou precies mee bezig was. Jaap en Sander, bedankt voor de vele gezellige (bord)spellensessies die we de afgelopen jaren hebben gehad en de gezamenlijke vakanties en lange weekenden weg per trein. Fijn dat jullie mij als paranimf willen bijstaan bij de verdediging. 



\section{About the Author}

Stefan Klootwijk was born on August 22, 1993, in Enschede, the Netherlands. After obtaining his high school diploma in 2011 Stefan started studying Applied Mathematics at the University of Twente. He obtained a bachelor's degree cum laude in 2014. Stefan continued with the master Applied Mathematics at the same institution, with a focus on the Mathematics of Operations Research. He obtained his master's degree cum laude in 2016, with a thesis on analysis of algorithms titled 'Probabilistic Analysis of Facility Location on Random Shortest Path Metrics'.

After obtaining his master's degree, Stefan immediately started his $\mathrm{PhD}$ research in the Discrete Mathematics and Mathematical Programming group at the University of Twente, under the supervision of dr. Bodo Manthey and prof.dr. Marc Uetz. During his PhD period he taught tutorial classes of the bachelor course Algebra and the honours course Linear Algebra and Coding Theory. His $\mathrm{PhD}$ research culminates with this thesis. 




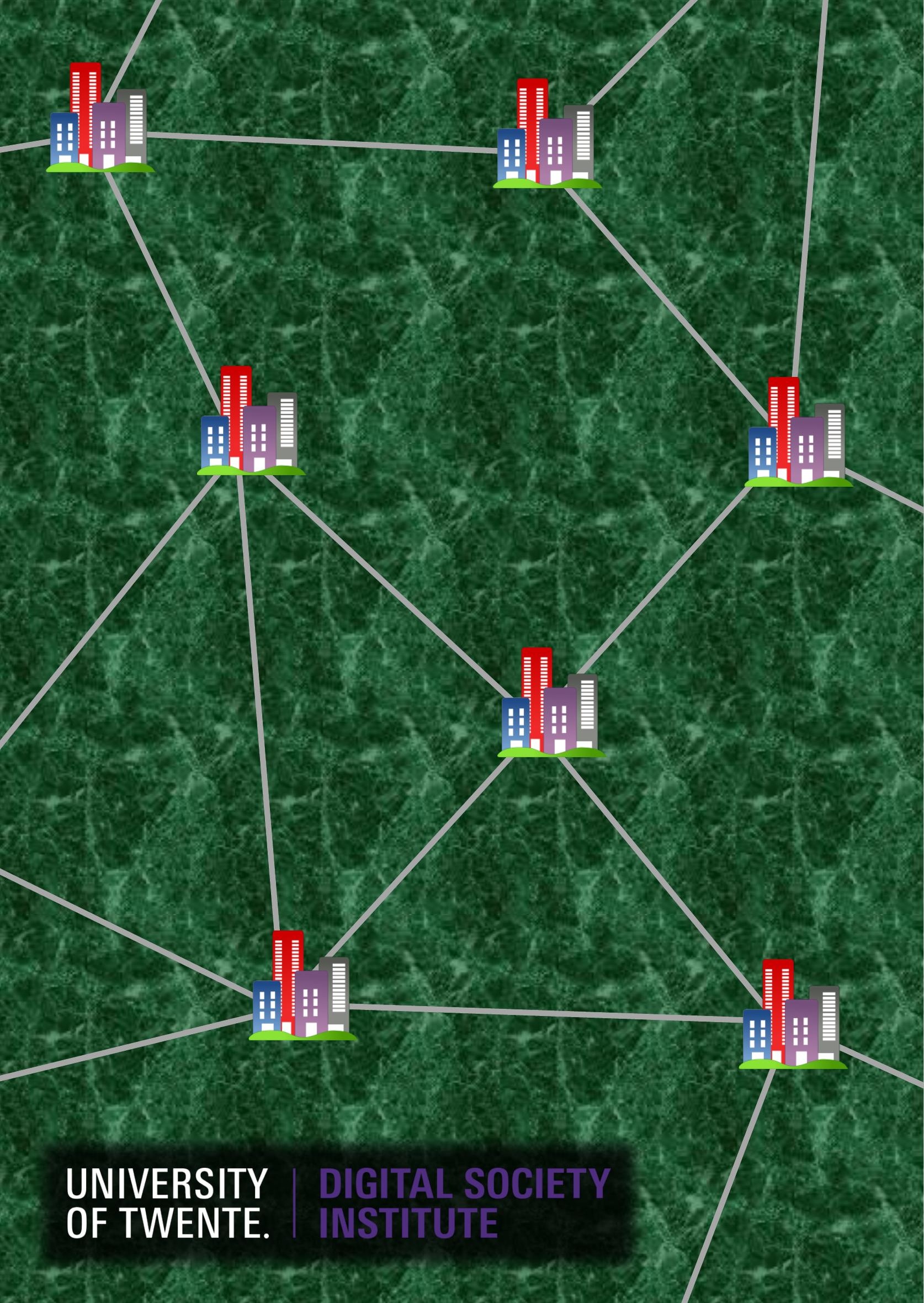

